\title{
Cobordism of singular maps
}

\author{
ANDRÁS SZÛ́CS
}

Throughout this paper we consider smooth maps of positive codimensions, having only stable singularities (see Arnold, Guseŭn-Zade and Varchenko [3, Section 1.4, Chapter 1]. We prove a conjecture, due to M Kazarian, connecting two classifying spaces in singularity theory for this type of singular maps. These spaces are: 1) Kazarian's space (generalising Vassiliev's algebraic complex and) showing which cohomology classes are represented by singularity strata. 2) The space $X_{\tau}$ giving homotopy representation of cobordisms of singular maps with a given list of allowed singularities as in work of Rimányi and the author [29;34;35].

We obtain that the ranks of cobordism groups of singular maps with a given list of allowed stable singularities, and also their $p$-torsion parts for big primes $p$ coincide with those of the homology groups of the corresponding Kazarian space. (A prime $p$ is "big" if it is greater than half of the dimension of the source manifold.) For all types of Morin maps (ie when the list of allowed singularities contains only corank 1 maps) we compute these ranks explicitly.

We give a very transparent homotopical description of the classifying space $X_{\tau}$ as a fibration. Using this fibration we solve the problem of elimination of singularities by cobordisms. (This is a modification of a question posed by Arnold [4, page 212].)

57R45, 55P42; 57R42, 55P15

In this paper all smooth maps will have positive codimensions and stable singularities. The aim of the present paper is to establish a close relationship between Kazarian's homological characteristic spectral sequence (generalising Vassiliev's complex) and the classifying space for cobordisms of maps having singularities only from a given fixed list (see the papers of the author [34] and the author with Rimányi [29]). Such a relationship was conjectured by M Kazarian, and it can be expressed as follows (in our formulation): There is a spectrum with a filtration such that the arising homological spectral sequence gives Kazarian's characteristic spectral sequence while the homotopy groups of the spectrum give the corresponding cobordism groups of singular maps (with a shift of the dimension). Hence the Hurewicz map for this spectrum induces a rational isomorphism from the cobordism groups of singular maps to the homology groups of Kazarian's space.

This allows us to extend quite a few classical theorems of cobordism theory to cobordisms of singular maps. In particular (at least modulo torsion): 
a) We extend the Pontrjagin-Thom theorem claiming that the characteristic numbers determine the cobordism class of a manifold.

b) We extend the definition of Conner and Floyd of the characteristic numbers of bordism classes and the statement that these numbers form a complete set of invariants. We give a complete and computable set of obstructions to elimination of singularities by cobordism.

c) For any set of corank one singularities we determine explicitly the ranks of the corresponding cobordism groups.

d) We give some general results beyond Morin maps, and also on the torsion groups.

e) We show a "Postnikov like tower" that produces the classifying space for cobordisms of singular maps as an iterated fibration. This gives a spectral sequence starting with the cobordism groups of immersions (of the singularity strata) and converging to the cobordism group of singular maps.

History To our knowledge cobordisms of singular maps were considered first by U Koschorke [24] and the author [34]. In 1984 Eliashberg [11] proved a PontrjaginThom type theorem for these cobordisms. For a long time there were hardly any more results except the paper of Chess [8] considering a similar but different problem. In the last decade works of Ando [1], Saeki [32], Ikegami and Saeki [18], Ikegami [17] and Kalmár [20] dealt with some cases of negative codimension maps. The subject of Sadykov's recent preprint [31] is closely related to our one, but applies a different approach; it generalises Ando's paper and gives a proof for the Pontrjagin-Thom construction for singular maps, different from our proof.

Acknowledgements Endre Szabó spent countless hours discussing with the author the material of the present paper. He made a significant contribution to this paper by suggesting that the "key bundles" (Definition 109) might be bundles. The author gave talks on the subject of this paper once a week during a semester on a seminar from February to May of 2005, where the discussion with the members of the audience helped in clarifying the ideas. My particular thanks are due to Endre Szabó and András Némethi, and further to Gábor Lippner, Tamás Terpai and Boldizsár Kalmár. 


\section{Part I Preliminaries}

\section{Cobordism groups}

Definition 1 By singularity we mean an equivalence class of germs $\left(\mathbb{R}^{c}, 0\right) \longrightarrow$ $\left(\mathbb{R}^{c+k}, 0\right)$ of fixed codimension $k>0$, where the equivalence is generated by $\mathcal{A}-$ equivalence (= left-right equivalence) and suspension: ${ }^{1}$

$$
\Sigma \eta(u, t)=(\eta(u), t),
$$

ie $\eta:\left(\mathbb{R}^{c}, 0\right) \rightarrow\left(\mathbb{R}^{c+k}, 0\right)$ is equivalent to

$$
\Sigma \eta=\eta \times \mathrm{id}_{\mathbb{R}^{1}}:\left(\mathbb{R}^{c} \times \mathbb{R}^{1}, 0\right) \rightarrow\left(\mathbb{R}^{c+k} \times \mathbb{R}^{1}, 0\right) .
$$

For a germ $\eta$ we denote by $[\eta]$ its equivalence class, and will call it also the singularity class of $\eta$. We say that the germ $\eta:\left(\mathbb{R}^{c}, 0\right) \rightarrow\left(\mathbb{R}^{c+k}, 0\right)$ has an isolated singularity at the origin if at no point near the origin the germ of $\eta$ is equivalent to that at the origin. Such an $\eta$ will be called the root of its singularity class $[\eta]$ and will be denoted by $\eta^{0}$. The root of $[\eta]$ is characterised also by having the smallest possible dimension $c$ in its class. Given a smooth map $f: M^{n} \rightarrow P^{n+k}$ we say that $x \in M^{n}$ is an [ $\left.\eta\right]$-point (or simply an $\eta$-point) if the germ of $f$ at $x$ belongs to $[\eta]$. The set of such points in $M^{n}$ will be denoted by $\eta(f)$. This is a submanifold (possibly nonclosed subset) in $M^{n}$ of dimension $n-c$. By this reason $c$ will be called the codimension of $\eta$.

In this paper we shall always consider stable singularities (see Arnol'd, Guseřn-Zade and Varchenko [3]) of maps of a fixed positive codimension $k$. For these type of singularities there is a natural hierarchy. We say that $\eta$ is more complicated than $\xi$, and write $\xi<\eta$, if in any neighbourhood of an $\eta$-point there is a $\xi$-point.

\section{Example 2}

1) The class of germs of regular (ie nonsingular) maps $\Sigma^{0}$ is the lowest "singularity."

2) The class of a fold germ (ie a stable $\Sigma^{1,0}$ germ) is lower than that of a cusp germ (ie of the stable $\Sigma^{1,1}$ germ).

\section{Definition 3}

a) Let $\tau$ be a set of stable singularity classes of codimension $k>0$. Given two smooth manifolds $M^{n}$ and $P^{n+k}$ of dimensions $n$ and $n+k$ respectively we say that a proper smooth map $f: M^{n} \rightarrow P^{n+k}$ is a $\tau$-map if at any point $x \in M^{n}$ the germ of $f$ belongs to a singularity class from $\tau$.

${ }^{1}$ also called trivial extension; see Kazarian [23]. 
b) If $M^{n}$ has nonempty boundary, then $P^{n+k}$ must have it too, and a $\tau$-map $f: M^{n} \rightarrow P^{n+k}$ must map $\partial M^{n}$ into $\partial P^{n+k}$, moreover $\partial M^{n}$ and $\partial P^{n+k}$ have collar neighbourhoods $\partial M^{n} \times[0, \varepsilon)$ and $\partial P^{n+k} \times[0, \varepsilon)$ such that $\left.f\right|_{\partial M^{n} \times[0, \varepsilon)}$ has the form $g \times \operatorname{id}_{[0, \varepsilon)}$, where $g: \partial M^{n} \rightarrow \partial P^{n+k}$ is a $\tau-\operatorname{map}, \operatorname{id}_{[0, \varepsilon)}$ is the identity map of $[0, \varepsilon)$.

Next we define manifolds with corners and then $\tau$-maps when both the source and the target are manifolds with corners.

Definition 4 We extend the definition of a smooth manifold with boundary to the definition of a smooth manifold with corners by allowing neighbourhoods of the type $\mathbb{R}^{n-m} \times[0, \varepsilon)^{m}$ for any $m \geq 0$. The numbers $n$ and $m$ will be called the dimension of the manifold and the codimension of the corner respectively. The positive codimension part (where $m>0$ ) will be called the boundary, its complement is the interior part of the manifold with corners. These parts will be denoted by $\partial$ and $\int$ respectively. Note that the boundary itself is an $n-1$-dimensional manifold with corners.

Now if $M^{n}$ and $P^{n+k}$ are manifolds with corners then a proper smooth map $f: M^{n} \rightarrow$ $P^{n+k}$ is a $\tau$-map if a neighbourhood of the corner of codimension $m$ of $M$ is mapped into that of $P^{n+k}$ by a map of the local form $g \times \operatorname{id}_{[0, \varepsilon)^{m}}$, where $g: \mathbb{R}^{n-m} \rightarrow \mathbb{R}^{n+k-m}$ is a $\tau$-map. So near a corner of codimension $m$ the map looks like an $m$-tuple suspension of a $\tau$-map; see Definition 1 .

Definition 5 For an arbitrary (possibly noncompact) oriented smooth $(n+k)$-dimensional manifold $P^{n+k}$ we will denote by $\operatorname{Cob}_{\tau}\left(P^{n+k}\right)$ the set of oriented $\tau$-cobordism classes of $\tau$-maps of $n$-dimensional manifolds in $P^{n+k}$. Namely, given two $\tau$-maps $f_{0}: M_{0}^{n} \rightarrow P^{n+k}$ and $f_{1}: M_{1}^{n} \rightarrow P^{n+k}$ (where $M_{0}^{n}, M_{1}^{n}$ are closed, oriented, smooth $n$-manifolds) they are said to be $\tau$-cobordant if there exist:

1) a compact, oriented $(n+1)$-manifold $W^{n+1}$ such that $\partial W^{n+1}=-M_{0}^{n} \sqcup M_{1}^{n}$,

2) a $\tau$-map $F: W^{n+1} \rightarrow P^{n+k} \times[0,1]$ such that

$$
F^{-1}\left(P^{n+k} \times\{i\}\right)=M_{i}^{n} \quad \text { and }\left.\quad F\right|_{M_{i}^{n}}=f_{i} \quad \text { for } i=0,1 .
$$

The $\tau$-cobordism class of a $\tau$-map $f$ will be denoted by $[f]$.

Note that for any two $n$-manifolds $N_{i}^{n}, i=0,1$, and any two $\tau$-maps $g_{i}: N_{i}^{n} \rightarrow P^{n+k}$ the disjoint union $g_{0} \sqcup g_{1}: N_{0}^{n} \sqcup N_{1}^{n} \rightarrow P^{n+k}$ is also a $\tau$-map. (The images of $g_{0}$ and $g_{1}$ may intersect.) The disjoint union defines an associative and commutative operation on $\operatorname{Cob}_{\tau}\left(P^{n+k}\right)$ with a null-element represented by the map of the empty set. 
Remark 6 In this paper we shall deal exclusively with oriented cobordisms of singular maps and so - typically - we shall not indicate that in our notation. (Each construction, definition, theorem of our paper has its unoriented counterpart, but we do not include them here.)

Definition 7 The notion $\operatorname{Cob}_{\tau}(P)$ of cobordism set of $\tau$-maps into $P$ can be extended to the case when $P$ is a finite simplicial complex. Let $P$ consist of a single simplex first. Note that a simplex is a manifold with corners in a natural way and also a cylinder over a simplex is that. Since we have defined the $\tau$-maps for manifolds with corners, the definition of $\operatorname{Cob}_{\tau}(P)$ can be given just repeating the definition for the manifold case (replacing the word "manifold" by "manifold with corners"). When $P$ consists of several simplices, then a $\tau$-map into $P$ is a set of $\tau$-maps into each simplex of (possibly empty) manifolds with corners, and with natural identifications of the corners and their maps corresponding to the identifications of the faces of the simplices. (Note that into each open simplex of dimension $i$ we map manifolds of dimension $i-k$.) The cobordism will be a $\tau$-map into the cylinder $P \times[0,1]$.

Remark 8 It is not obvious but true that for any finite simplicial complex $P$ the set $\operatorname{Cob}_{\tau}(P)$ with this operation forms a group, ie there is an inverse element. This fact follows of course from the homotopy representation of this set - the generalised Pontrjagin-Thom construction - proved in papers by Rimányi and the author [29], Ando [1] and Sadyakov [31], along with the present paper, but here we give an explicit geometric description of the inverse element.

\subsection{On the inverse element}

a) If $P^{n+k}=\mathbb{R}^{n+k}$, then composing a map $f: M^{n} \rightarrow \mathbb{R}^{n+k}$ with a reflection $r$ in a hyperplane of $\mathbb{R}^{n+k}$ and an orientation reversing diffeomorphism $T: M^{n} \rightarrow$ $M^{n}$ of the source one gets the inverse element: $-[f]=[r \circ f \circ T]$.

b) If $P^{n+k}$ has the form $N^{n+k-1} \times \mathbb{R}^{1}$, then still one can reflect in a "hyperplane", ie in a submanifold $N^{n+k-1} \times\{c\}, c \in \mathbb{R}^{1}$ and compose it with the orientation reversing map $T$ of the source we get the inverse.

c) For an arbitrary manifold $P^{n+k}$ we need the notion of "framed $\tau$-map":

Definition 9 A framed $\tau$-map from an $n$-manifold $M^{n}$ into an $(n+k+1)$-manifold $Q^{n+k+1}$ is a map germ along $M^{n} \times\{0\} \approx M^{n}$ of a $\tau$-map

$$
M^{n} \times \mathbb{R}^{1} \supset \mathcal{N}^{n+1} \stackrel{\tilde{f}}{\longrightarrow} Q^{n+k+1}
$$


defined in a neighbourhood $\mathcal{N}^{n+1}$ of $M^{n} \times\{0\}$, such that around each point $p=$ $(x, 0) \in M^{n} \times\{0\}$ and its image $q=\tilde{f}(p)$ there exist coordinate neighbourhoods $W_{x}^{n+1}, \widetilde{W}_{q}^{n+k+1}$ such that $W_{x}^{n+1}=U_{x} \times V_{0}$, where $U_{x} \subset M^{n}, V_{0} \subset \mathbb{R}^{1}, U_{x} \approx \mathbb{R}^{n}$, $V_{0} \approx \mathbb{R}^{1}$, and $\widetilde{W}_{q}^{n+k+1} \approx \mathbb{R}^{n+k+1}=\mathbb{R}^{n+k} \times \mathbb{R}^{1}$ in which $\tilde{f}$ has the form: $g \times \operatorname{id}_{\mathbb{R}^{1}}$, where $g: \mathbb{R}^{n} \rightarrow \mathbb{R}^{n+k}$ is a $\tau$-map and $\operatorname{id}_{\mathbb{R}^{1}}$ is the identity map $\mathbb{R}^{1} \rightarrow \mathbb{R}^{1}$. (Note that the $\mathbb{R}^{1}$-direction in the source is always directed along the second factor of $M^{n} \times \mathbb{R}^{1}$, while in the target there is no such a fixed direction.)

\section{Remark 10}

1) The framed $\tau$-map is an extension of the notion of "immersion with a normal vector field" to the case of maps with singularities.

2) Analogously it can be defined the notion of $\ell$-framed $\tau$-maps as germs at $M^{n} \times 0$ of maps $M^{n} \times \mathbb{R}^{\ell} \supset \mathcal{N}^{n+\ell} \rightarrow Q^{n+k+\ell}$, etc.

Example 11 If $f: M^{n} \rightarrow P^{n+k}$ is a $\tau$-map, then the germ of the product map $f \times \operatorname{id}_{\mathbb{R}^{1}}: M^{n} \times \mathbb{R}^{1} \rightarrow P^{n+k} \times \mathbb{R}^{1}$ along $M^{n} \times\{0\}$ gives a framed $\tau$-map of $M^{n}$ into $P^{n+k} \times \mathbb{R}^{1}$.

Definition 12 The cobordism set of framed $\tau$-maps of closed, (oriented) smooth $n$-manifolds into an (oriented) smooth $(n+k+1)$-dimensional manifold $Q^{n+k+1}$ can be defined by an obvious modification of the definition of $\tau$-cobordism. The set of cobordism classes will be denoted by $\operatorname{Cob}_{\tau \oplus 1}\left(Q^{n+k+1}\right)$. The cobordism set of $\ell$-framed $\tau$-maps will be denoted by $\operatorname{Cob}_{\tau \oplus \ell}\left(Q^{n+k+1}\right)$.

Proposition 13 Given oriented $n$ and $n+k$-dimensional manifolds $M^{n}$ and $P^{n+k}$ associating to a $\tau$-map $f: M^{n} \rightarrow P^{n+k}$ the $\ell$-framed $\tau$-map $f \times \operatorname{id}_{\mathbb{R}^{\ell}}$ one obtains a one-to-one correspondence

$$
\operatorname{Cob}_{\tau}\left(P^{n+k}\right) \stackrel{1-1}{\longrightarrow} \operatorname{Cob}_{\tau \oplus \ell}\left(P^{n+k} \times \mathbb{R}^{\ell}\right)
$$

for any natural number $\ell$. (Recall, that $k>0$.)

Proof This will be given later; see Remark 46. It will follow from a stratified version of the "Compression Theorem" (see Rourke and Sanderson [30] and also Gromov [13]). The manifold $P^{n+k}$ can be replaced by any finite simplicial complex.

Now for an element in $\operatorname{Cob}_{\tau}\left(P^{n+k}\right)$ its inverse can be obtained using the one-to-one correspondence in the Proposition, since in $\operatorname{Cob}_{\tau \oplus 1}\left(P^{n+k} \times \mathbb{R}^{1}\right)$ we have a target manifold of the type b). More explicitly for a $\tau$-map $f: M^{n} \rightarrow P^{n+k}$ a representative 
of the inverse cobordism class $-[f]$ can be obtained as follows: Consider the germ of $f \times \mathrm{id}_{\mathbb{R}^{1}}: M^{n} \times \mathbb{R}^{1} \rightarrow P^{n+k} \times \mathbb{R}^{1}$ at $M^{n} \times 0$. This can be visualised as the map

$$
\tilde{f}: M^{n} \stackrel{f}{\longrightarrow} P^{n+k} \hookrightarrow P^{n+k} \times \mathbb{R}^{1}
$$

with an "upward" directed vector field $\uparrow$. (The lines $\{p\} \times \mathbb{R}^{1} \subset P^{n+k} \times \mathbb{R}^{1}, p \in P^{n+k}$, with the positive direction we call upward directed lines.) Now according to b) we reflect this map $\tilde{f}$ in the "hyperplane" $P^{n+k} \times\{0\}$ in order to obtain a representative of the inverse cobordism class $-[\tilde{f}]$. Since the image of $\tilde{f}$ lies in $P^{n+k} \times\{0\}$, this reduces to taking the same map $\tilde{f}$ with a downward directed vector field $\downarrow$. The "Stratified Compression Theorem" (see Theorem 1) will claim that there is an isotopy $\varphi_{t}$ of $P^{n+k} \times \mathbb{R}^{1}$ such that $\varphi_{0}$ is the identity map, and the differential $d\left(\varphi_{1}\right)$ maps the downward directed vector field along $\tilde{f}$ into the upward directed one along $\varphi_{1} \circ \tilde{f}$. Now $\varphi_{1} \circ \tilde{f} \circ T: M^{n} \rightarrow P^{n+k} \times \mathbb{R}^{1}$ can be composed with a projection to $P^{n+k}$, and this gives a $\tau$-map $g: M^{n} \rightarrow P^{n+k}$ such that $[g]=-[f]$ in $\operatorname{Cob}_{\tau}\left(P^{n+k}\right)$.

Finally if $P$ is an arbitrary finite simplicial complex, then we will use the relative version of the Stratified Compression Theorem to get an "isotopy" of $P$ moving each simplex over itself and turning the downward directed vector field into an upward directed one.

Another way of reducing the case when $P$ is a finite simplicial complex to that when it is a manifold is the following:

A finite simplicial complex is the deformation retract of its very small neighbourhood in a big dimensional Euclidean space. This neighbourhood can be chosen so that its closure is a smooth manifold with boundary. Now having a $\tau$-map in the simplicial complex $P$ it extends to a $\tau$-map into a small closed neighbourhood $U$ of $P$ in the Euclidean space, where $U$ is a manifold with boundary. Applying the procedure constructing the inverse element in $\operatorname{Cob}_{\tau}(U)$ and then restricting this element to $P$ we obtain the inverse element of the class of the original $\tau$-map.

In order to show that $\operatorname{Cob}_{\tau}()$ is a contravariant functor we have to define the induced maps. This will be done if we show how to pull back a $\tau$-map.

Proposition 14 (Pulling back a $\tau$-map) $\operatorname{Cob}_{\tau}(P)$ is a contravariant functor on the category of finite simplicial complexes and their simplicial maps. (Actually it can be extended to their continuous maps.)

Proof 1) Let $P^{n+k}$ and $P^{n^{\prime}+k}$ be first smooth manifolds of dimensions $n+k$ and $n^{\prime}+k$ respectively. Let $g: P^{n^{\prime}+k} \rightarrow P^{n+k}$ be a continuous map. We show that $g$ induces a well-defined homomorphism $g^{*}: \operatorname{Cob}_{\tau}\left(P^{n+k}\right) \rightarrow \operatorname{Cob}_{\tau}\left(P^{n^{\prime}+k}\right)$. Let 
$f: M^{n} \rightarrow P^{n+k}$ be a $\tau$-map. The map $g$ can be approximated by a smooth map (homotopic to $g$ ) transverse to the image of each singularity stratum of $f$, we will denote this transverse map again by $g$. Let us define the "pullback" map $g^{*}(f)$ as follows. Consider the map $\widetilde{f}: \widetilde{M}^{n} \rightarrow P^{n^{\prime}+k}$, where

$$
\widetilde{M}^{n}=\left\{\left(p^{\prime}, m\right) \in P^{n^{\prime}+k} \times M^{n} \mid g\left(p^{\prime}\right)=f(m)\right\}, \quad \widetilde{f}\left(p^{\prime}, m\right)=p^{\prime}
$$

and put $g^{*}(f)=\tilde{f}$. The map $g^{*}(f)$ will be a $\tau$-map. (This was formulated without proof by Kazarian [23, page 71]. The proof of this statement is contained implicitly in the paper by Rimányi and the author [29]. Below we shall prove it.) Further the cobordism class of the obtained map $\tilde{f}$ will be shown to be independent of the chosen transverse approximation of $g$. Moreover we show that homotopic maps induce the same homomorphisms, ie $g \cong h \Longrightarrow g^{*}=h^{*}$.

\section{Claim 15}

a) $\widetilde{M}^{n}$ is a smooth manifold.

b) $\tilde{f}$ is a $\tau$-map.

Proof It was shown in the proof of Theorem 1 in [29] (see also the author's papers [38] and [39] for Morin maps) that in a neighbourhood of the $\eta$-stratum the map $f$ has local form

$$
\eta \times \operatorname{id}_{\mathbb{R}^{u}}: \mathbb{R}^{c} \times \mathbb{R}^{u} \rightarrow \mathbb{R}^{c+k} \times \mathbb{R}^{u} .
$$

Let us call these local coordinates canonical.

Here the subspaces $\{0\} \times R^{u}$ are directed along the singularity stratum and its image, while the complementary subspaces $\mathbb{R}^{c} \times\{0\}$ and $\mathbb{R}^{c+k} \times\{0\}$ are directed along the normal fibres of the stratum $\eta(f)$ in the source and that of its image $\tilde{\eta}(f)=f(\eta(f))$ in the target respectively. Since the map $g$ is transverse to the stratum $\tilde{\eta}(f)$ for any point $p^{\prime} \in P^{n^{\prime}+k}$ such that $g\left(p^{\prime}\right)=f(x)$, where $x \in \eta(f)$, there is a local coordinate system $\mathbb{R}^{c+k} \times \mathbb{R}^{v}$ centred around $p^{\prime}$, such that in these local coordinates and in the canonical local coordinates around $g\left(p^{\prime}\right)$ the map $g$ has the form $\operatorname{id}_{\mathbb{R}^{c+k}} \times h: \mathbb{R}^{c+k} \times \mathbb{R}^{v} \rightarrow$ $\mathbb{R}^{c+k} \times \mathbb{R}^{u}$, where $h: \mathbb{R}^{v} \rightarrow \mathbb{R}^{u}$ is a smooth map. Now it is clear that in the given local coordinates the map $\tilde{f}=g^{*}(f): \mathbb{R}^{c} \times \mathbb{R}^{v} \rightarrow \mathbb{R}^{c} \times \mathbb{R}^{u}$ has the form $\operatorname{id}_{\mathbb{R}^{c} \times h}$. In particular $\widetilde{M}^{n}$ is a smooth manifold (because the arbitrarily chosen point $\left(x, p^{\prime}\right)$ has a Euclidean neighbourhood and the change of coordinates is smooth since they are such in $M^{n}$ and $P^{n^{\prime}+k}$ ). Further $\tilde{f}$ has in these local coordinates a normal form equivalent to $\eta$ around the point $\left(x, p^{\prime}\right)$. Hence the pulled back map is a $\tau$-map. 
2) All this works also when $P^{n+k}$ and $P^{n^{\prime}+k}$ are not manifolds but those with corners. Then the map $g$ must map corners of codimension $r$ in $P^{n^{\prime}+k}$ into those in $P^{n+k}$. (Of course in this case $\widetilde{M}^{n}$ will be a manifold with corners.)

3) Finally let $P$ and $P^{\prime}$ be any finite simplicial complexes. We have defined the groups $\operatorname{Cob}_{\tau}(P)$ and $\operatorname{Cob}_{\tau}\left(P^{\prime}\right)$ for this case too. Recall that each simplex is considered as a manifold with corners, and $\tau$-maps in this case are those into each simplex in a compatible way. Each simplicial map can be considered as a map of manifolds with corners. Hence $g^{*}: \operatorname{Cob}_{\tau}(P) \rightarrow \operatorname{Cob}_{\tau}\left(P^{\prime}\right)$ is defined.

4) If $P$ is a simplicial complex and $P_{1}$ is its subdivision, then there are natural isomorphisms $\operatorname{Cob}_{\tau}(P) \rightarrow \operatorname{Cob}_{\tau}\left(P_{1}\right)$ and $\operatorname{Cob}_{\tau}\left(P_{1}\right) \rightarrow \operatorname{Cob}_{\tau}(P)$.

The first is obtained by taking transversal intersections of the image of the $\tau$-maps with the new faces in $P_{1}$. The second map is obtained by noticing that at the points of the omitted faces the $\tau$-maps can be attached along their corners and get an interior point of the image.

5) If $g$ is any continuous map of the simplicial complex $P^{\prime}$ into the simplicial complex $P$, then we can choose an appropriate subdivision of $P^{\prime}$ and replace $g$ by a homotopic simplicial map.

6) For homotopic maps $g \cong h$ from $P^{\prime}$ to $P$ the induced maps $g^{*}$ and $h^{*}$ from $\operatorname{Cob}_{\tau}(P) \rightarrow \operatorname{Cob}_{\tau}\left(P^{\prime}\right)$ will coincide.

Indeed, given a $\tau$-map in $P$ the homotopy between $g$ and $h$ also can be made transverse to each stratum $\tilde{\eta}(f)$. Pulling back the map $f$ by this transverse homotopy we obtain a $\tau$-cobordism between the two pullbacks $g^{*}(f)$ and $h^{*}(f)$.

Corollary 16 Given two finite simplicial complexes $P$ and $P^{\prime}$ denote by $\left[P^{\prime}, P\right]$ the set of homotopy classes of continuous maps $P^{\prime} \rightarrow P$. Then a map arises

$$
\left[P^{\prime}, P\right] \times \operatorname{Cob}_{\tau}(P) \rightarrow \operatorname{Cob}_{\tau}\left(P^{\prime}\right)
$$

that is additive in its second factor.

\section{Remark 17}

a) Above we described the extension of the group-valued functor $\operatorname{Cob}_{\tau}(-)$ from smooth manifolds to (finite) simplicial complexes. This extended functor satisfies the conditions of the Brown representability theorem. Hence there is a space $X_{\tau}$ such that: $\operatorname{Cob}_{\tau}(P)=\left[P, X_{\tau}\right]$ for any finite simplicial complex $P$ [36]. (Here 
the brackets [, ] denote the set of homotopy classes. The space $X_{\tau}$ has been constructed in [29] explicitly for more general types of maps.) ${ }^{2}$

b) Since $\operatorname{Cob}_{\tau}(P)$ is actually a group, the space $X_{\tau}$ will be an $H$-group according to an addendum to Brown's theorem.

c) The space $X_{\tau}$ is a loop space, ie there is a space $Z_{\tau}$ such that $X_{\tau}$ is homotopy equivalent to $\Omega Z_{\tau}$. Indeed, if we denote by $Z_{\tau}$ the classifying space of framed $\tau$-maps, then for any finite simplicial complex $P$ the group of framed $\tau$-maps into the suspension $S P$ is isomorphic to the $\operatorname{group~} \operatorname{Cob}_{\tau}(P)$ (by Proposition 13). Hence

$$
\left[P, X_{\tau}\right]=\left[S P, Z_{\tau}\right] \text { for any } P
$$

On the other hand $\left[S P, Z_{\tau}\right]=\left[P, \Omega Z_{\tau}\right]$ and so $X_{\tau} \cong \Omega Z_{\tau}$ since the representing space of a functor is homotopically unique. (Note that this shows again that $X_{\tau}$ is an $H$-group - since it is a loop-space - and $\operatorname{Cob}_{\tau}(P)$ is a group.)

d) The above procedure can be iterated and we obtain that $X_{\tau}$ is an infinite loop space, ie for each $\ell$ there is a space $Z_{\tau}^{\ell}$ such that $X_{\tau}=\Omega^{\ell} Z_{\tau}^{\ell}$. Here $Z_{\tau}^{\ell}$ will be the classifying space of $\ell$-framed $\tau$-maps.

In the introduction we promised to establish a relationship between the classifying space of cobordism of singular maps with prescribed singularities - this is the space $X_{\tau}$ - and Kazarian's space (which we will denote by $\mathcal{K}_{\tau}$ ). In the next section we recall the two definitions of this space $\mathcal{K}_{\tau}$. The first is described by Kazarian in [22]. (The referee informed me that it is due to Thom; see Haefliger and Kosiński [14].) The second was communicated to me - without proof - by Kazarian [21]. We show the equivalence of these two definitions; this equivalence will be used in the concrete computations.

\footnotetext{
${ }^{2}$ The definition of $\tau$-maps in [29] was different from that here. It included both local restrictions (as here) and global ones (these are not considered here). In the presence of global restrictions the set of $\tau$-maps does not form a group, the classifying space is not an $H$-space and finally there is no such a nice connection with the Kazarian space that we are going to prove here. In general the situation is much more difficult in the presence of global restrictions. We can illustrate this by comparing the classification of embeddings with that of immersions (these are actually special cases of maps with global restrictions and maps with only local restrictions.) While immersion theory is completely reduced to algebraic topology, embedding theory is hopeless in general.
} 


\section{Part II Kazarian's space}

\section{Definition derived from the Borel construction}

\subsection{Unstable version}

Here we construct a "preliminary", "unstable" version of Kazarian's space. ${ }^{3}$ Let $\tau$ be a set of stable singularity classes such that each of them is $K$-determined for a big enough number $K$. Put $V_{n}=J_{0}^{K}\left(\mathbb{R}^{n}, \mathbb{R}^{n+k}\right)$ the space of $K$-jets of germs $\left(\mathbb{R}^{n}, 0\right) \rightarrow\left(\mathbb{R}^{n+k}, 0\right)$ at the origin. Let us denote by $G_{n}$ the group $J_{0}^{K}\left(\operatorname{Diff}^{+}\left(\mathbb{R}^{n}, 0\right)\right) \times$ $J_{0}^{K}\left(\right.$ Diff $\left.^{+}\left(\mathbb{R}^{n+k}, 0\right)\right)$ ie the group of $K$-jets of orientation preserving diffeomorphismgerms of $\mathbb{R}^{n}$ and $\mathbb{R}^{n+k}$ at the origin. Those $K$-jets in $V_{n}$ which belong to the same singularity class, form a $G_{n}$-orbit. In particular the $K$-jets in the singularity classes lying in $\tau$ form a $G_{n}$-invariant subset in $V_{n}$, which we denote by $V_{\tau}^{n}$. Put $\mathcal{K}_{\tau}^{\prime}(n)=V_{\tau}^{n} \times_{G_{n}} E G_{n}$. Let us denote by $\pi^{\prime}$ the fibration

$$
\pi^{\prime}: \mathcal{K}_{\tau}^{\prime}(n) \stackrel{V_{\tau}^{n}}{\longrightarrow} B G_{n} .
$$

If $[\eta] \in \tau$ is a singularity class from $\tau$, then we denote by $B \eta(n)$ the corresponding subset in $\mathcal{K}_{\tau}^{\prime}(n)$. (For any fibre $V_{\tau}^{n}$ the intersection $V_{\tau}^{n} \cap B \eta(n)$ coincides with the set of germs $\mathcal{A}_{+}$-equivalent to $\eta$. Here $\mathcal{A}_{+}$-equivalent means $\mathcal{A}$-equivalent through orientation preserving diffeomorphisms.)

Lemma 18 (Haefliger and Kosiński [14] and Kazarian [22])

a) Let $f: M^{n} \rightarrow P^{n+k}$ be any $\tau$-map. Let us denote by $\eta(f)$ the subset of $M^{n}$, where the germ of $f$ is in $[\eta]$. Then there is a map $\kappa_{f}^{\prime}: M^{n} \rightarrow \mathcal{K}_{\tau}^{\prime}(n)$ such that $\left(\kappa_{f}^{\prime}\right)^{-1}(B \eta(n))=\eta(f)$ for any $[\eta] \in \tau$.

b) Moreover this map $\kappa_{f}^{\prime}$ has the following extra property. First we note that the space $B G_{n}$ is homotopy equivalent to $B S O_{n} \times B S O_{n+k}$. Let us lift the universal vector bundles over $B S O_{n}$ and $B S O_{n+k}$ to $B G_{n}$. The extra property of the map $\kappa_{f}^{\prime}$ is that, the composition $\pi^{\prime} \circ \kappa_{f}^{\prime}$ pulls back the bundles $T M^{n}$ and $f^{*} T P^{n+k}$ from these lifted bundles respectively, where $T M^{n}$ and $T P^{n+k}$ denote the tangent bundles over $M^{n}$ and $P^{n+k}$, respectively.

Remark 19 Actually Kazarian has constructed this map $\kappa_{f}^{\prime}: M^{n} \rightarrow \mathcal{K}_{\tau}^{\prime}(n)$, we recall his construction in the sketch of the proof below. By the Kazarian map $\kappa_{f}^{\prime}$ we shall mean this particular construction (or its stabilised version described later).

\footnotetext{
3 "unstable" means that in this subsection we do not consider yet the identification of the germs with their suspensions; see Definition 1.
} 
Remark 20 The space $\mathcal{K}_{\tau}^{\prime}(n)$ is not yet the final definition of the Kazarian space. It still depends on the dimension $n$. The final definition will be obtained in Section 2.3, after some stabilisation.

Definition 21 Pull back the universal bundles $\gamma_{n}^{S O} \rightarrow B S O_{n}$ and $\gamma_{n+k}^{S O} \rightarrow B S O_{n+k}$ to the space $\mathcal{K}_{\tau}^{\prime}(n)$ and consider their formal difference as a virtual $k$-dimensional vector bundle. This bundle will be denoted by $v_{\tau}^{k}(n)$ (or sometimes simply by $v(n)$ ) and called the $n$-universal virtual normal bundle for $\tau$-maps.

Sketch of the proof of Lemma 18 (See Kazarian [22].) Let us fix Riemannian metrics on $M^{n}$ and $P^{n+k}$ and denote by $T^{\varepsilon} M^{n}$ the set of tangent vectors of length less than $\varepsilon$. There is a fibrewise map $\hat{f}: T^{\varepsilon} M^{n} \rightarrow f^{*} T P^{n+k}$ defined on a neighbourhood of the zero section of $T M^{n}$ such that $\exp _{P}^{n+k} \circ \hat{f} \circ \exp _{M}^{-1}=f$, where exp denotes the exponential map. Let $V_{\tau}^{n}\left(M^{n}\right)$ denote the subspace of $J_{0}^{K}\left(T M^{n}, f^{*} T P^{n+k}\right) \rightarrow M^{n}$ corresponding to the singularities in $\tau$. So $V_{\tau}^{n}\left(M^{n}\right)$ is the total space of a fibration over $M^{n}$ with fibre $V_{\tau}^{n}$ and structure group $G_{n}$. The map $\hat{f}$ defines a section $\tilde{f}$ of this bundle. The bundle

$$
V_{\tau}^{n}\left(M^{n}\right) \stackrel{V_{\tau}^{n}}{\longrightarrow} M^{n}
$$

can be induced from the bundle

$$
K_{\tau}^{\prime}(n) \stackrel{V_{\tau}^{n}}{\longrightarrow} B G_{n}
$$

The obtained map $V_{\tau}^{n}\left(M^{n}\right) \longrightarrow K_{\tau}^{\prime}(n)$ composed with the section $\tilde{f}$ gives the map $\kappa_{f}^{\prime}: M^{n} \rightarrow \mathcal{K}_{\tau}^{\prime}(n)$. Clearly $\left(\kappa_{f}^{\prime}\right)^{-1}(B \eta(n))=\eta(f)$.

\subsection{Addendum to the Borel construction}

Let $G$ be a compact group Lie, let $V$ be a contractible smooth manifold with smooth (left) $G$-action on it, and let $B V=E G \times{ }_{G} V$ be the Borel construction applied to $V$. Further let $\Sigma$ be an orbit of $G$ in $V$, let $x$ be a point in $\Sigma$, and let $G_{x}$ be the stabiliser of $x$. (Hence $\Sigma=G / G_{x}$.) Finally let $S_{x}$ be a small transverse slice of $\Sigma$ at $x$. We can choose a Riemannian metric on $V$ such that the $G$-action will be isometric. Choose $S_{x}$ orthogonal to $\Sigma$. Then $G_{x}$ acts on the tangent space $T S_{x}$ orthogonally. Let us denote by $\rho_{x}$ the arising representation of $G_{x}$ on $T S_{x}$.

\section{Lemma 22}

a) The subset $B \Sigma=E G \times_{G} \Sigma$ in $B V=E G \times_{G} V$ is the classifying space $B G_{x}$ of the stabiliser group $G_{x}$. 
b) A neighbourhood of $B \Sigma$ in $B V$ can be identified with the universal bundle over $B G_{x}$ associated with the representation $\rho_{x}$.

Proof a) This part was proved by Kazarian [22; 23]. Below our proof for part b) will show it too.

b) Let $\mathcal{N}$ be a tubular $G$-invariant neighbourhood of $\Sigma$ (in $V$ ) fibred over $\Sigma$ with fibre $S_{g x}$ over $g x \in \Sigma$. Note that for any $g \in G$ we obtain a bijection from $S_{x}$ to $S_{g x}$. Nevertheless the fibration $\mathcal{N} \rightarrow \Sigma$ might be nontrivial since different elements $g, g^{\prime}$ of $G$ might give different bijections onto the same fibre $S_{g x}$ if $g x=g^{\prime} x$.

Let us lift the bundle $\mathcal{N} \rightarrow \Sigma=G / G_{x}$ to $G$ pulling it back by the projection $G \rightarrow$ $G / G_{x}$. Let $\widetilde{\mathcal{N}}$ be the obtained bundle over $G$. We claim that the bundle $\widetilde{\mathcal{N}} \rightarrow G$ is trivial. Indeed, let $\widetilde{S}_{g}$ be the fibre over $g \in G$. Then by the definition of the pullback bundle there is an identification of $\widetilde{S}_{g}$ with $S_{[g]}$, where $[g] \in G / G_{x}$ is the coset of $g$. ( $[g] \in G / G_{x}$ is identified with the point $g x \in \Sigma$.) Since each element $g \in G$ gives a bijection $S_{x} \rightarrow S_{g x}$, we obtain a canonical identification $\widetilde{S}_{e} \rightarrow \widetilde{S}_{g}$ of the fibre over the neutral element $e \in G$ with the fibre over $g \in G$. So we get a $G$-action on $\widetilde{\mathcal{N}}$ and the obtained decomposition $\widetilde{\mathcal{N}}=G \times \widetilde{S}_{e}$ is $G$-equivariant (with trivial $G$-action on $\widetilde{S}_{e}$.)

The fibration $\widetilde{\mathcal{N}} \rightarrow \mathcal{N}$ with fibre $G_{x}$ is $G$-equivariant. The well-known Borel construction is a functor from the category of $G$-spaces and $G$-equivariant maps to the category of topological spaces and their maps. Applying the Borel construction to the equivariant map $\widetilde{\mathcal{N}} \rightarrow \mathcal{N}$ we obtain a map $\widetilde{\mathcal{N}} \times_{G} E G \rightarrow \mathcal{N} \times_{G} E G$. Here the domain is $\widetilde{\mathcal{N}} \times_{G} E G=G \times_{G}\left(\widetilde{S}_{e} \times E G\right)=\widetilde{S}_{e} \times E G$. The target $\mathcal{N} \times_{G} E G$ is a neighbourhood of $B \Sigma$ in $B V$. The map itself is the quotient map factorising out by the diagonal $G_{x}$-action on $\widetilde{S}_{e} \times E G$. Hence the neighbourhood $B \mathcal{N}$ of $B \Sigma$ in $B V$ can be identified with $\widetilde{S}_{e} \times_{G_{x}} E G$, where $\widetilde{S}_{e}$ is a ball, and $G_{x}$ acts on it by the representation $\rho_{x}$.

Later (in Lemma 35) we shall need the following simple Remark.

Remark 23 Let $V, \Sigma, G$ be as above. Let $F \subset V$ be transverse to $\Sigma$. We identify $V$ with a fibre of the Borel construction $B V$. Then $F$ is transverse to $B \Sigma$ in $B V$.

\subsection{Stabilisation}

Until this point in the construction of the space $K_{\tau}^{\prime}(n)$ we did not use that forming the equivalence classes of germs we identified each germ with its suspension. Now we are going to use it. Note that adding an arbitrary smooth vector bundle $F$ over 
$M^{n}$ to both $T M^{n}$ and $f^{*} T P^{n+k}$, and replacing the map $\hat{f}: T^{\varepsilon} M \rightarrow f^{*}(T P)$ by $\hat{f} \oplus \operatorname{id}_{F}$, where $\operatorname{id}_{F}$ is the identity map of $F$, we obtain the same stratification on $M^{n}$ by singularity types. In other words, at $x \in M^{n}$ the $K$-jet of the map $\left(\hat{f} \oplus \mathrm{id}_{F}\right)_{x}: T_{x}^{\varepsilon} M^{n} \oplus F_{x} \rightarrow T_{f(x)} P^{n+k} \oplus F_{x}$ is equivalent to the (dim $\left.F_{x}\right)$-tuple suspension of the $K$-jet of $\widehat{f}: T_{x} M^{n} \rightarrow T_{f(x)} P^{n+k}$. For $x \in M^{n}$ the following equivalences hold: $x \in \eta(f) \Leftrightarrow x \in \eta(\widehat{f}) \Leftrightarrow x \in \eta\left(\widehat{f} \oplus \mathrm{id}_{F}\right)$. Now choose the bundle $F$ to be equal to the (stable) normal bundle of $M^{n}$ in $\mathbb{R}^{N}$ for a big enough $N(N \geq$ $2 n+2: F=v_{M} . T M^{n} \oplus v_{M} \approx \varepsilon^{N}$ ). Consider the fibrewise polynomial maps of degree at most $K$ and with zero constant term from $\varepsilon^{N}=M^{n} \times \mathbb{R}^{N}$ into $f^{*} T P^{n+k} \oplus v_{M}$, ie the bundle $J_{0}^{K}\left(\varepsilon^{N}, f^{*} T P^{n+k} \oplus v_{M}\right) \rightarrow M^{n}$ with fibre $J_{0}^{K}\left(\mathbb{R}^{N}, \mathbb{R}^{N+k}\right)$ (= polynomial maps from $\left(\mathbb{R}^{N}, 0\right)$ to $\left(\mathbb{R}^{N+k}, 0\right)$ of degree at most $\left.K\right)$. Again in each fibre we can take only those polynomial maps which have at the origin a singularity type belonging to $\tau$, ie the subspace $V_{\tau}^{N}$. The bundle $J_{0}^{K}\left(\varepsilon^{N}, f^{*} T P^{n+k} \oplus v_{M}\right) \rightarrow M^{n}$ can be induced from the bundle $J_{0}^{K}\left(\varepsilon^{N}, \gamma_{N+k}^{S O}\right) \rightarrow B S O_{N+k}$.

Definition 24 Denote by $\mathcal{K}_{\tau}(N)$ the subspace in $J_{0}^{K}\left(\varepsilon^{N}, \gamma_{N+k}^{S O}\right)$ such that the intersection of $\mathcal{K}_{\tau}(N)$ with any fibre $J_{0}^{K}\left(\mathbb{R}^{N}, \mathbb{R}^{N+k}\right)$ is $V_{\tau}^{N}$.

The inclusion $S O_{N} \subset S O_{N+1}$ induces a map $\mathcal{K}_{\tau}(N) \rightarrow \mathcal{K}_{\tau}(N+1)$. Finally put $\mathcal{K}_{\tau}=\lim _{N \rightarrow \infty} \mathcal{K}_{\tau}(N)$ and call this space Kazarian space for $\tau$-maps.

Definition 25 The lift of the $n$-universal virtual normal bundle $v(n)$ (see Definition 21 ) to the space $\mathcal{K}_{\tau}$ will be denoted by $v=v_{\tau}^{k}$ and called the universal virtual normal bundle of $\tau$-maps.

Remark 26 Note that there is a partition of $\mathcal{K}_{\tau}$ according to the singularity classes in $\tau$, ie $\mathcal{K}_{\tau}=\bigcup_{[\eta] \in \tau} B \eta$ and the analogue of Lemma 18 holds; see the Appendix (Section 20).

\section{Definition of the Kazarian space by gluing}

For this second definition we will need the notion of "global normal form" of a singularity type $[\eta]$ found in $[38 ; 39 ; 29]$, hence we recall it now. Let $\eta:\left(\mathbb{R}^{c}, 0\right) \rightarrow$ $\left(\mathbb{R}^{c+k}, 0\right)$ be the root of the singularity type $[\eta]$. (Recall that this means that $c$ is the minimal possible dimension for the representatives of $[\eta]$.) Let $G_{\eta}^{O}$ denote the maximal compact subgroup of the automorphism group of $\eta$ (in the sense of Jänich [19]; see also Wall [44] and Rimányi and the author [29]). Let $\lambda_{\eta}$ and $\tilde{\lambda}_{\eta}$ be the representations of $G_{\eta}^{O}$ in the source and the target of $\eta$, respectively (ie for $\forall g \in G_{\eta}^{O}, \tilde{\lambda}_{\eta}(g) \circ \eta \circ \lambda_{\eta}^{-1}(g)=\eta$. 
Since we will consider always oriented source and target manifolds we will need only the "orientation preserving" subgroup of $G_{\eta}^{O}$, ie the group $G_{\eta}$ defined as follows: $\left.G_{\eta}=\left\{g \in G_{\eta}^{O} \mid \operatorname{det} \lambda(g) \cdot \operatorname{det} \tilde{\lambda}(g)>0\right\}\right)$.

Definition 27 The "global normal form" of $\eta$ consists of

1) the vector bundle $\xi_{\eta}=E G_{\eta} \times \lambda_{\eta} \mathbb{R}^{c} \stackrel{\mathbb{R}^{c}}{\longrightarrow} B G_{\eta}$,

2) the vector bundle $\tilde{\xi}_{\eta}=E G_{\eta} \times \tilde{\lambda}_{\eta} \mathbb{R}^{c+k} \stackrel{\mathbb{R}^{c+k}}{\longrightarrow} B G_{\eta}$,

3) the (nonlinear) fibrewise map $\Phi_{\eta}: \xi_{\eta} \longrightarrow \widetilde{\xi}_{\eta}$ having the form $\left(\operatorname{id}_{E G_{\eta}} \times \eta\right) / G_{\eta}$, where $\operatorname{id}_{E G_{\eta}}$ denotes the identity map of the contractible space $E G_{\eta}$.

Explanation The map $\operatorname{id}_{E G_{\eta}} \times \eta$ is a $G_{\eta}$-equivariant map from $E G_{\eta} \times \mathbb{R}^{c}$ into $E G_{\eta} \times \mathbb{R}^{c+k}$. The map $\Phi_{\eta}$ is obtained quotienting out by the action of $G_{\eta}$.

Proposition $28 \quad[37 ; 29]$ Let $M^{n}$ and $P^{n+k}$ be oriented manifolds of dimensions $n$ and $n+k$ respectively. Suppose that $f: M^{n} \rightarrow P^{n+k}$ is a proper $\tau-$ map, and $\left.f\right|_{\eta(f)}$ is an embedding into $P^{n+k}$. Let us denote by $T$ and $\widetilde{T}$ the tubular neighbourhoods of $\eta(f)$ in $M^{n}$ and $f(\eta(f))$ in $P^{n+k}$, respectively. Then there is a commutative diagram

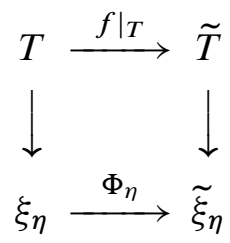

where the vertical maps are vector bundle morphisms, which are isomorphisms on the fibres.

Remark 29 This proposition shows that the universal map $\Phi_{\eta}$ describes the $\tau$-map $f$ in the tubular neighbourhood of the whole stratum $\eta(f)$, that is why it was called a "global normal form".

Remark 30 The relative version of the above Proposition also holds, with the same proof as in [29]. This means the following: Suppose that the vertical arrows of (1) are given on $\left.T\right|_{A}$ and $\left.\widetilde{T}\right|_{A}$ for some closed subset $A \subset \eta(f)$, so that the diagram is commutative, where the maps are defined, and the pair $(\eta(f), A)$ is a relative $\mathrm{CW}$ complex. Then these given vertical arrows can be extended to have the commutative diagram (1). 
Now we give another description of the Kazarian space by a gluing procedure. In order to avoid the confusion of the two definitions we denote this space by $g l \mathcal{K}_{\tau} .{ }^{4}$

Let $[\eta]$ be a top singularity type in $\tau$ and put $\tau^{\prime}=\tau \backslash\{[\eta]\}$. Suppose that the space $g l \mathcal{K}_{\tau^{\prime}}$ has been constructed and it has been shown that $g l \mathcal{K}_{\tau^{\prime}}$ is homotopically equivalent to the space $\mathcal{K}_{\tau}^{\prime}$. Let us denote by $v^{\prime}: g l \mathcal{K}_{\tau^{\prime}} \rightarrow \mathcal{K}_{\tau^{\prime}}$ the constructed homotopy equivalence and by $u^{\prime}$ we denote its homotopy inverse. (The starting step of the induction will be given at the end of the proof.) Let $B G_{\eta}$,fin be a finite dimensional manifold approximating the classifying space $B G_{\eta}$. (For example on the Stiefel manifold $V_{c}\left(R^{N}\right)$ of $c$-tuples of vectors in $R^{N}$ there is a free $G_{\eta}$-action, and this manifold is $N-c-1-$ connected, where $c$ is the codimension of the singularity $\eta$. So we can put $B G_{\eta, \text { fin }}=V_{c}\left(\mathbb{R}^{N}\right) / \lambda_{\eta}\left(G_{\eta}\right)$.)

Let us denote the restrictions of the bundles $\xi_{\eta}$ and $\widetilde{\xi}_{\eta}$ to $B G_{\eta \text {,fin }}$ by $\xi_{\eta \text {,fin }}$ and $\widetilde{\xi}_{\eta \text {,fin }}$ respectively, and their disc bundles by $D \xi_{\eta \text {,fin }}$ and $D \widetilde{\xi}_{\eta \text {,fin }}$. Finally let $\Phi=\Phi_{\eta \text {,fin }}$ denote the restriction of $\Phi_{\eta}$ to $D \xi_{\eta \text {,fin }}$; see Diagram (1). (The disc bundles are chosen so that $D \xi_{\eta \text {,fin }}=\Phi_{\eta}^{-1}\left(\widetilde{D} \xi_{\eta \text {,fin }}\right)$.) By Remark 26 the map $\Phi: D \xi_{\eta \text {,fin }} \rightarrow \widetilde{D} \xi_{\eta \text {,fin }}$ defines a map $\kappa_{\Phi}: D \xi_{\eta \text {,fin }} \rightarrow \mathcal{K}_{\tau}$. This map carries a point $x \in D \xi_{\eta \text {,fin }}$ into such a point of $\mathcal{K}_{\tau}$ that corresponds to a singularity of the same type as the germ of $\Phi$ at $x$. Hence the preimage of the $\eta$-stratum $B \eta \subset \mathcal{K}_{\tau}$ is the zero section of the bundle $D \xi_{\eta \text {,fin }}$. Finally let us denote by $\kappa_{\Phi}^{S}$ the restriction of the map $\kappa_{\Phi}$ to the sphere bundle $S\left(\xi_{\eta, \text { fin }}\right)=\partial D\left(\xi_{\eta, \text { fin }}\right)$.

Remark 31 It follows from Remark 26 that $\kappa_{\Phi}$ pulls back from the universal virtual normal bundle $v_{\tau}$ (see Definition 25) the virtual bundle $\pi_{D\left(\xi_{\eta}\right)}^{*}\left(\widetilde{\xi}_{\eta}-\xi_{\eta}\right)$ where $\pi_{D\left(\xi_{\eta}\right)}$ is the projection $D\left(\xi_{\eta}\right) \rightarrow B G_{\eta}$.

Definition 32 We define a finite dimensional approximation of the space $g l \mathcal{K}_{\tau}$ as follows:

$$
g l \mathcal{K}_{\tau, \text { fin }} \stackrel{\text { def }}{=} g l \mathcal{K}_{\tau^{\prime}, \text { fin }} \bigcup_{u^{\prime} \circ \kappa_{\Phi}^{S}} D \xi_{\eta, \text { fin }}=g l \mathcal{K}_{\tau^{\prime}} \sqcup D \xi_{\eta, \text { fin }} / x \sim u^{\prime} \circ \kappa_{\Phi}^{S}(x),
$$

for all $x \in S\left(\xi_{\eta, \text { fin }}\right)$, ie in the disjoint union of $g l \mathcal{K}_{\tau}^{\prime}$ and $D \xi_{\eta \text {,fin }}$ we identify each $x \in S\left(\xi_{\eta, \text { fin }}\right)$ with its image $u^{\prime} \circ \kappa_{\Phi}^{S}(x) \in g l \mathcal{K}_{\tau^{\prime}}$. Recall that $u^{\prime}: \mathcal{K}_{\tau^{\prime}} \rightarrow g l \mathcal{K}_{\tau^{\prime}}$ is a homotopy equivalence, that exists by the assumption of the induction. Its homotopy inverse is $v^{\prime}$.

\footnotetext{
${ }^{4}$ The space $g l \mathcal{K}_{\tau}$ will not be homeomorphic to the space $\mathcal{K}_{\tau}$, but it will be homotopically equivalent. Moreover $g l \mathcal{K}_{\tau}$ is a deformation retract in $\mathcal{K}_{\tau}$.
} 
Definition 33 Let $v: g l \mathcal{K}_{\tau} \rightarrow \mathcal{K}_{\tau}$ be a continuous map with the following properties:

a) $\left.v\right|_{g l \mathcal{K}_{\tau^{\prime}}} \cong v^{\prime}$

b) $v \mid D\left(\xi_{\eta}\right)=\kappa_{\Phi}$

Such a map exists, because on the sphere bundle $S\left(\xi_{\eta}\right)$ the two maps on the right hand sides of a) and b) are homotopic.

Claim 34 The map $v$ is a homotopy equivalence.

Proof It is enough to show that the map

$$
\widehat{\kappa}_{\Phi}:\left(D\left(\xi_{\eta}\right), S\left(\xi_{\eta}\right)\right) \rightarrow\left(\mathcal{K}_{\tau}, \mathcal{K}_{\tau^{\prime}}\right)
$$

defined by $\kappa_{\Phi}$ induces isomorphisms of the homology groups.

Lemma $35 \kappa_{\Phi}$ maps each fibre of the bundle $D\left(\xi_{\eta}\right) \rightarrow B G_{\eta}$ into a slice transverse to $B \eta$ in $\mathcal{K}_{\tau}$.

Postponing the proof of this Lemma we prove Claim 34. After an isotopy it can be supposed that $\kappa_{\Phi}$ maps each fibre of $D\left(\xi_{\eta}\right)$ into a fibre of the normal bundle $v_{\eta}$ of the stratum $B \eta$ in $\mathcal{K}_{\tau}$. Let $g: B G_{\eta} \rightarrow B \eta$ be the map defined by the map $\kappa_{\Phi}$ mapping the zero section of $\xi_{\eta}$ into that of $v_{\eta}$. Hence $g^{*}\left(v_{\eta}\right)=\xi_{\eta}$. On the other hand $\xi_{\eta}$ is the universal bundle with structure group $G_{\eta}$, and $v_{\eta}$ admits the group $G_{\eta}$ as structure group. Hence there is a map $h: B \eta \rightarrow B G_{\eta}$ such that $h^{*}\left(\xi_{\eta}\right)=v_{\eta}$. We obtain that the composition map $h \circ g: B G_{\eta} \rightarrow B G_{\eta}$ is homotopic to the identity map (because $(h \circ g)^{*}\left(\xi_{\eta}\right)=\xi_{\eta}$ and the inducing map is homotopically unique). Since the homology groups of $B G_{\eta}=B \eta$ are finitely generated Abelian groups in each dimension we obtain that the homomorphisms induced by the maps $h$ and $g$ are isomorphisms. Let us denote by $\hat{g}$ the map of the corresponding Thom spaces induced by $g$, ie $\hat{g}: T \xi_{\eta} \rightarrow T v_{\eta}$. By the Thom isomorphism $\hat{g}$ also induces isomorphism of the homology groups, since so does $g$. But the homology groups of the Thom spaces $T \xi_{\eta}$ and $T v_{\eta}$ are canonically isomorphic to those of the pairs $\left(D\left(\xi_{\eta}\right), S\left(\xi_{\eta}\right)\right)$ and $\left(\mathcal{K}_{\tau}, \mathcal{K}_{\tau^{\prime}}\right)$ respectively, and identifying the corresponding homology groups using these canonical isomorphisms the homomorphism $\widehat{g}_{*}$ turns into $\widehat{\kappa}_{\Phi *}$. Now by the five lemma the map $v$ induces isomorphism of the homology groups. This proves Claim 34 .

Proof of Lemma 35 Let us use Remark 23 with the substitutions:

a) $V=J_{0}^{K}\left(\mathbb{R}^{N}, \mathbb{R}^{N+k}\right)$, ie $V$ is the space of polynomial maps $\mathcal{P}=\mathcal{P}^{K}\left(\mathbb{R}^{N}, \mathbb{R}^{N+k}\right)$ from $\mathbb{R}^{N}$ into $\mathbb{R}^{N+k}$ of degree (at most) $K$ having zero constant term. 
b) $\quad \Sigma=[\eta] \cap \mathcal{P}$. This means the following: Let $\eta: \mathbb{R}^{c} \rightarrow \mathbb{R}^{c+k}$ be a polynomial map of degree (at most) $K$ giving the root of the stable singularity class $[\eta]$. Then $\Sigma$ consists of all the polynomial maps from $\mathcal{P}$ equivalent to $\eta$ in the sense of Definition 1.

The map $\kappa_{\Phi}$ restricted to a fibre $\mathbb{R}^{c}$ can be described as follows: $a \in \mathbb{R}^{c}$ is mapped into the polynomial map $f_{a}$ in $\mathcal{P}$ such that: if $x \in \mathbb{R}^{c}, y \in \mathbb{R}^{N-c}$ and $\mathbb{R}^{N}=\mathbb{R}^{c} \times \mathbb{R}^{N-c}$, then $f_{a}(x, y)=((\eta(x-a)-\eta(a)), y)$. By Remark 23 it is enough to show that the image of $\mathbb{R}^{c}$ is transverse to $[\eta] \cap \mathcal{P}$ in $\mathcal{P}$. ( $F$ from Remark 23 will be here $\kappa_{\Phi}\left(\mathbb{R}^{c}\right)$.) If $\mathcal{U}$ is a small enough neighbourhood of $f_{0}$ in $\mathcal{P}$ then for each $g \in \mathcal{U}$ a linear subspace $L_{g}$ can be defined in $\mathbb{R}^{N}$ such that $L_{g}$ contains $\operatorname{ker} d g$ and $\operatorname{dim} L_{g}=\operatorname{dim} d f_{0}$. We denote by $\ell$ the dimensions of these spaces $L_{g}$. Again if $\mathcal{U}$ is small enough then all the spaces $L_{g}$ can be projected isomorphically onto $L_{f_{0}}$, and so they can be identified with $\mathbb{R}^{\ell}$ in a continuous way.

We define a map

$$
\Pi: \mathcal{U} \rightarrow \mathcal{P}^{K}\left(\mathbb{R}^{\ell}, \mathbb{R}^{N+k}\right)
$$

by taking the restriction of each $g \in \mathcal{U}$ to $L_{g}$. Note that $\Pi(g)$ is either the genotype of $g$ or a trivial extension (ie a multiple suspension) of it. ${ }^{5}$

Further clearly $\Pi$ is a submersion onto its image. A theorem of Mather says that two stable germs $f$ and $g$ are $\mathcal{A}$ equivalent if and only if their genotypes are contact equivalent. From this it follows that the preimage of the contact orbit of $\Pi\left(f_{0}\right)$ is the $\mathcal{A}$ orbit of $f_{0}$.

$\Pi\left(\kappa_{\Phi}\left(\mathbb{R}^{c}\right)\right)$ is the deformation of the genotype of $f_{0}$, which is the same as the deformation of the genotype of $\eta$ given by $\eta$. Again by a theorem of Mather such a deformation is transverse to the contact orbit of the genotype if the germ itself (in this case $\eta$ ) is $\mathcal{A}$ stable. Hence $\Pi\left(\kappa_{\Phi}\left(\mathbb{R}^{c}\right)\right)$ is a transverse slice to the contact orbit of the genotype of $f_{0}$. But then the lift of this slice (ie $\kappa_{\Phi}\left(\mathbb{R}^{c}\right)$ ) is transverse to the preimage of the contact orbit, which is precisely the $\mathcal{A}$ orbit of $f$.

The start of the induction of the proof of the statement $g l \mathcal{K}_{\tau} \cong \mathcal{K}_{\tau}$ : Recall that the simplest "singularity type" $\left[\eta_{0}\right]$ is always the class of maps of maximal rank $\Sigma^{0}$.

We show that for $\tau=\left\{\left[\eta_{0}\right]\right\}$ both spaces $\mathcal{K}_{\tau}$ and $g l \mathcal{K}_{\tau}$ are homotopy equivalent to the Grassmann manifold $B S O(k)$. We denote these spaces here by $\mathcal{K}_{0}$ and $g l \mathcal{K}_{0}$ respectively.

1) By the gluing procedure we have to consider the root of codimension $k$ maps of maximal rank, this is the germ $(0,0) \hookrightarrow\left(\mathbb{R}^{k}, 0\right)$. The automorphism group of this germ is the group of germs of all diffeomorphisms $\operatorname{Diff}\left(\mathbb{R}^{k}, 0\right)$. Its maximal compact

${ }^{5}$ Recall that the genotype of the germ $g$ can be defined as its restriction to the kernel of its differential. 
subgroup is $G_{\eta_{0}}^{O}=O(\tilde{\alpha})$. The oriented version of this group $G_{\eta_{0}}$ is $S O(k)$. The representations $\lambda$ and $\tilde{\lambda}$ on the source $(0)$ and on the target $\left(\mathbb{R}^{k}\right)$ respectively are the trivial map $\lambda: S O(k) \rightarrow\{1\}$ and the standard inclusion $\widetilde{\bar{\lambda}}: S O(k) \rightarrow O(k)$. Now the space $g l \mathcal{K}_{0}(k)$ is $E S O(k) \times S O(k)\{0\}=B S O(k)$.

2) By the Borel construction we obtain the following: Let us denote by $\mathcal{P}_{\eta_{0}}^{K}$ the space of polynomial maps $\mathbb{R}^{N} \succ \mathbb{R}^{N+k}$ of degree (at most) $K$ having differentials of maximal rank at the origin. The group $K$-jets of germs of diffeomorphisms of $\mathbb{R}^{N+k}$ acts on this space. Associating to each polynomial map and to each germ of diffeomorphisms their linear parts at the origin we obtain an equivariant homotopy equivalence from $\mathcal{P}_{\eta_{0}}^{K}$ to the Stiefel manifold $V_{N}\left(\mathbb{R}^{N+k}\right)$ provided with the action of the linear group $G L^{+}\left(\mathbb{R}^{N+k}\right)$. Replacing the linear group by the homotopically equivalent orthogonal group we obtain that

$$
\mathcal{K}_{0}=\lim _{N \rightarrow \infty} V_{N}\left(\mathbb{R}^{N+k}\right) \times_{S O(N+k)} E S O(N+k) .
$$

Since $V_{N}\left(\mathbb{R}^{N+k}\right)=S O(N+k) / S O(k)$ we conclude

$$
\mathcal{K}_{0}(k)=\lim _{N \rightarrow \infty} E S O(N+k) / S O(k)=B S O(k) .
$$

From now on we will use the notation $\mathcal{K}_{\tau}$ for the space $g l \mathcal{K}_{\tau}$. In particular we will use the decomposition $\mathcal{K}_{\tau}=\bigcup_{i} D \xi_{\eta_{i}}$.

\section{The Kazarian spectral sequence}

Let $\eta_{0}<\eta_{1}<\ldots$ be a linear ordering of the singularities in $\tau$ compatible with the hierarchy of singularities. Let $\tau_{i}$ be the set $\left\{\eta_{j} \mid j \leq i\right\}$ and denote $\mathcal{K}_{\tau_{i}}$ by $\mathcal{K}_{i}, G_{\eta_{i}}$ by $G_{i}, \xi_{\eta_{i}}$ by $\xi_{i}$ etc. Let us consider the homological spectral sequence arising from the filtration $\mathcal{K}_{0} \subset \mathcal{K}_{1} \subset \cdots \subset \mathcal{K}_{\tau}$. Then $E_{p, q}^{1}=H_{p+q}\left(T \xi_{q}\right)$, where $\xi_{q}=\xi_{\eta_{q}}$ and $T \xi_{q}$ is the Thom space. By the Thom isomorphism

$$
E_{p, q}^{1} \approx H_{p+q-c_{q}}\left(B G_{q} ; \widetilde{\mathbb{Z}}\left(\xi_{q}\right)\right),
$$

where $c_{q}$ is the dimension of the bundle $\xi_{q}$, the coefficients $\widetilde{\mathbb{Z}}\left(\xi_{q}\right)$ are the integers twisted by the orientation of $\xi_{q}$. This spectral sequence converges to the group $H_{*}\left(\mathcal{K}_{\tau}\right)$, ie $\bigoplus_{p+q=n} E_{p, q}^{\infty}$ is associated to $H_{n}\left(\mathcal{K}_{\tau}\right){ }^{6}$

\footnotetext{
${ }^{6}$ Kazarian's ordering is different from ours.
} 


\section{Part III Pontrjagin-Thom construction for $\tau$-maps}

In our proof of Pontrjagin-Thom construction for $\tau$-maps we follow the method of Wells [45] reducing the cobordisms of immersions to those of embeddings with many normal vector fields by lifting immersions into a high dimensional space. (This method is completely different from that of [29], not to mention [1] or [31].)

\section{Definition of $\tau$-embedding}

Definition 36 Let $\tau$ be a set of stable singularity classes of codimension $k>0$. A quintuple $\left(M^{n}, Q^{q}, e, V, \mathcal{F}\right)$ is called a $\tau$-embedding if the following holds:

1) $M^{n}$ and $Q^{q}$ are manifolds (possibly with nonempty boundaries) of dimensions $n$ and $q$ respectively.

2) $e: M^{n} \hookrightarrow Q^{q}$ is an embedding. (If $M^{n}$ has boundary, then $Q^{q}$ has it too, $e^{-1}\left(\partial Q^{q}\right)=\partial M^{n}$, and $e$ is transverse to the boundary of $Q^{q}$.)

3) $V=\left\{v_{1}, \ldots, v_{N}\right\}$ is a finite set of linearly independent vector fields along $e\left(M^{n}\right)$ (ie sections of the bundle $\left.\left.T Q^{q}\right|_{e\left(M^{n}\right)}\right)$. Here $N=q-n-k$. We shall identify $V$ with the $N$-dimensional trivialised subbundle of $\left.T Q^{q}\right|_{e\left(M^{n}\right)}$ generated by the vector fields. We require that the vector fields on the boundary $e\left(\partial M^{n}\right)$ be tangent to $\partial Q^{q}$.

4) $\mathcal{F}$ is a foliation of dimension $N$ on a neighbourhood of $e\left(M^{n}\right)$, and it is tangent to $V$ along $e\left(M^{n}\right)$.

5) In a neighbourhood of any point $p \in M^{n}$ the composition of the embedding $e$ with the local projection along the leaves of the foliation $\mathcal{F}$ gives a local map (onto a small $n+k$-dimensional slice transverse to the leaves), having at $p$ a singularity belonging to $\tau$.

\section{Remark 37}

1) Note that $N=\operatorname{dim} Q^{q}-\operatorname{dim} M^{n}-k$. When the codimension of the $\tau-$ embedding is not clear from the context, then we have to include $N$ in the notation. In this case we shall write $(\tau, N)$-embedding for the above defined $\tau$-embedding.

2) The quintuple $\left(M^{n}, Q^{q}, e, V, \mathcal{F}\right)$ often will be denoted simply by $e$, and we shall say that $e$ is a $\tau$-embedding, 
Example 38 Let $f: M^{n} \rightarrow P^{n+k}$ be a $\tau$-map, let $g: M^{n} \hookrightarrow \mathbb{R}^{N}$ be an embedding and $Q^{q}=P^{n+k} \times \mathbb{R}^{N}, q=n+k+N$. Put $e=f \times g$ and $V=\left\{v_{1}, \ldots, v_{N}\right\}$, where $v_{1}, \ldots, v_{N}$ are the vector fields arising from a basis in $\mathbb{R}^{N}$. Let $\mathcal{F}$ be the vertical foliation with the leaves $\{x\} \times \mathbb{R}^{N}$. Then the quintuple $\left(M^{n}, Q^{q}, e, V, \mathcal{F}\right)$ is a $\tau$-embedding.

Definition 39 In $P^{n+k} \times \mathbb{R}^{N}$ we shall call the above defined vector fields $v_{1}, \ldots, v_{N}$ vertical.

Remark 40 If $\left(M^{n}, Q, e, V, \mathcal{F}\right)$ is a $\tau$-embedding, where $Q=P^{n+k} \times \mathbb{R}^{N}$ and $V=\left\{v_{1}, \ldots, v_{N}\right\}$ are the vertical basis vector fields, $\mathcal{F}$ is formed by the leaves $\{p\} \times R^{N}$, for $p \in P^{n+k}$, then the composition $f=\pi \circ e$ is a $\tau$-map $f: M^{n} \rightarrow P^{n+k}$ , where $\pi: P^{n+k} \times \mathbb{R}^{N} \rightarrow P^{n+k}$ is the projection.

Remark 41 Given a $\tau$-embedding $\left(M^{n}, Q, e, V, \mathcal{F}\right)$ a stratification arises on the manifold $M^{n} ; M^{n}=\bigcup\{\eta(\pi \circ e) \mid[\eta] \in \tau\}$ according to the singularity types of the composition of $e$ with the local projection $\pi$ along the leaves. Sometimes we shall write $\eta(e)$ instead of $\eta(\pi \circ e)$.

\section{The Compression Theorem for stratified manifolds}

Definition 42 (Gromov [13]) Let $\mathcal{S}$ be a finite stratification on a compact manifold $M^{n}: M^{n}=S_{1} \cup \cdots \cup S_{i}, S_{j}$ are the strata, $S_{j} \subset \bar{S}_{j-1}$. Let $e$ be an embedding of $M^{n}$ in a manifold $Q^{q}, q=n+k+N$ (with the usual assumption on the boundary) and let $v_{1}, \ldots, v_{N}$ be linearly independent vector fields along $e\left(M^{n}\right)$ (at $e\left(\partial M^{n}\right)$ they are tangent to $\left.\partial Q^{q}\right)$. For $x \in S_{j}$ denote by $V_{x}$ the vector space generated by the vectors $v_{1}, \ldots, v_{N}$ at $e(x)$. So $V_{x} \subset T_{e(x)} Q^{q}$. Let $d e$ be the differential of the embedding $e$. We say that the vector fields $v_{1}, \ldots, v_{N}$ are transverse to the stratification $\mathcal{S}$ if for any $x \in M^{n}$ the space $V_{x}$ and the $d e$-image $d e\left(T_{x} S_{j}\right)$ of the tangent space of the stratum $S_{j}$ at $x$ intersect only in the origin of the space $T_{e(x)} Q^{q}$.

\section{Theorem 1 (Stratified Compression Theorem, SCT)}

a) Let $M^{n}$ be a compact smooth manifold, $P^{n+k}$ be an arbitrary smooth $(n+k)-$ manifold, $e: M^{n} \hookrightarrow P^{n+k} \times \mathbb{R}^{N}$ an embedding. Let $\mathcal{S}$ be a finite stratification of $M^{n}: M^{n}=S_{1} \cup \cdots \cup S_{i}$. Further let $v_{1}, \ldots, v_{\ell}, \ell \leq N$, be vector fields along $e$ and transverse to the stratification $\mathcal{S}$. Then there exists an isotopy $\varphi_{t}: P^{n+k} \times \mathbb{R}^{N} \rightarrow P^{n+k} \times \mathbb{R}^{N}, t \in[0,1]$, such that $\varphi_{0}$ is the identity map, and the vector fields $d \varphi_{1}\left(v_{1}\right), \ldots, d \varphi_{1}\left(v_{\ell}\right)$ are vertical vector fields along the embedding $\varphi_{1} \circ e$, (see Definition 39). 
b) (Relative version) Suppose we are in the previous conditions and $K \subset M^{n}$ is a compact in $M^{n}$ such that on a neighbourhood of $K$ the vector fields $v_{1}, \ldots, v_{\ell}$ are vertical. Then the isotopy $\varphi_{t}$ can be chosen so that it keeps fixed the points of a neighbourhood of $K$.

Proof of b) Induction on $i$ (= the number of strata). For $i=1$ this is the usual Compression Theorem for immersions; see Rourke and Sanderson [30]. It can be supposed that the vertical vector fields are given not only along the submanifold $e\left(M^{n}\right)$ but also along a tubular neighbourhood of $e\left(M^{n}\right)$ in $P^{n+k} \times R^{N}$ and are tangent to the leaves of the foliation. (Such an extension always exists and it is homotopically unique.) Now suppose that the vector fields are vertical already on a neighbourhood $U$ (in $P^{n+k} \times \mathbb{R}^{N}$ ) of the union of the $e$-images of the strata: $S_{i} \cup S_{i-1} \cup \cdots \cup S_{i-j}$. (Recall that $\operatorname{dim} S_{i}<\operatorname{dim} S_{i-1}<\cdots<\operatorname{dim} S_{i-j}<\cdots<\operatorname{dim} S_{1}=n$.) Let us choose neighbourhoods $U^{\prime}, U^{\prime \prime}$ of the union $S_{i} \cup \cdots \cup S_{i-j} \cup K$ such that $U^{\prime \prime} \subset \bar{U}^{\prime \prime} \subset$ $U^{\prime} \subset \bar{U}^{\prime} \subset U$. Put $M^{*}=M^{n} \backslash U^{\prime \prime}$ and $K^{*}=\left(M^{n} \backslash U^{\prime \prime}\right) \cap \bar{U}^{\prime}$. Then $M^{*}$ is stratified by the strata $S_{1} \cup \cdots \cup S_{i-j-1}$ intersected with $M^{*}$. The vector fields are made vertical on $U$, in particular on $K^{*}$. By the relative version of the inductional assumption we can make the vector fields vertical on $M^{*}$ (or even on a neighbourhood of it in $P^{n+k} \times \mathbb{R}^{N}$ ) by an isotopy fixed on $K$. So we get vector fields vertical on the whole $M^{n}$.

Now we show that starting with a $(\tau, N)$-embedding in $P^{n+k} \times \mathbb{R}^{N}$ obtained as a result of the isotopy described in the previous Lemma (ie the vector fields are vertical along $e\left(M^{n}\right)$ ) a further isotopy (fixed on $e\left(M^{n}\right)$ ) can be applied that will turn the foliation vertical in a neighbourhood of $e\left(M^{n}\right)$. (Recall that the foliation is called vertical if its leaves coincide with the sets $\{p\} \times \mathbb{R}^{N}$ in a neighbourhood of $e\left(M^{n}\right)$.)

Lemma 43 Let $\left(M^{n}, P^{n+k} \times \mathbb{R}^{N}, e, V, \mathcal{F}\right)$ be a $(\tau, N)$-embedding such that the vector fields $v_{1}, \ldots, v_{N}$ along $e\left(M^{n}\right)$ are vertical, ie coincide with the standard vector fields. Then there is an isotopy $\psi_{t}$ of $P^{n+k} \times \mathbb{R}^{N}$ such that $\psi_{0}$ is the identity map, $\psi_{1}(\mathcal{F})=\mathcal{F}^{\prime}$, where $\mathcal{F}^{\prime}$ denotes the vertical foliation formed by the leaves $\{x\} \times \mathbb{R}^{N}, x \in P^{n+k}$, in a neighbourhood of $e\left(M^{n}\right)$. Moreover, $d \psi_{1}\left(v_{j}\right)=v_{j}^{\prime}$ for $j=1, \ldots, N$, where $\left\{v_{1}^{\prime}, \ldots v_{N}^{\prime}\right\}$ denote the standard vertical vector fields arising from the standard basis of $\mathbb{R}^{N}$.

Definition 44 Let us call the subsets $P^{n+k} \times\{y\}$ in $P^{n+k} \times \mathbb{R}^{N}$, where $y \in \mathbb{R}^{N}$, horizontal sections.

Proof We start with two general remarks: 
1) If $L$ and $L^{\prime}$ are two subsets of $P^{n+k} \times \mathbb{R}^{N}$ such that they intersect each horizontal section $P^{n+k} \times\{y\}$ for $y \in U \subset \mathbb{R}^{N}$ in exactly one point, then a one-to-one map $L \rightarrow L^{\prime}$ arises.

2) If $A$ is a subset in $P^{n+k} \times \mathbb{R}^{N}$, and for each $a=(p, y), a \in A$, subsets $L_{a}$ and $L_{a}^{\prime}$ are given such that $a \in L_{a}, a \in L_{a}^{\prime}$ and $L_{a}, L_{a}^{\prime}$ intersect each horizontal section $P^{n+k} \times\left\{y^{\prime}\right\}$ for $y^{\prime}$ close to $y$ in a unique point, then a family of maps $L_{a} \rightarrow L_{a}^{\prime}$ arises. Suppose that $L_{a_{1}} \cap L_{a_{2}}=\varnothing$ and $L_{a_{1}}^{\prime} \cap L_{a_{2}}^{\prime}=\varnothing$ for $a_{1} \neq a_{2}$. Then a one-toone continuous map $\alpha: \bigcup_{a \in A} L_{a} \rightarrow \bigcup_{a \in A} L_{a}^{\prime}$ arises by associating to each other the points on the same horizontal section. If $A, L_{a}, L_{a}^{\prime}$ are smooth submanifolds so that $\left\{\bigcup L_{a} \mid a \in A\right\}$ and $\left\{\bigcup L_{a}^{\prime} \mid a \in A\right\}$ are also smooth submanifolds, then the map $\alpha$ is a smooth map.

Now let $V^{\perp}$ be the subbundle in $\left.T\left(P^{n+k} \times \mathbb{R}^{N}\right)\right|_{e\left(S_{i}\right)}$ formed by the orthogonal complement of $\left.V\right|_{e\left(S_{i}\right)} \oplus T e\left(S_{i}\right)$. (Recall that $S_{i}$ is the smallest dimensional stratum.) Let $\exp \left(V^{\perp}\right)$ be the image of $V^{\perp}$ at the exponential map. Choose for $A$ a small neighbourhood of $e\left(S_{i}\right)$ in $\exp \left(V^{\perp}\right)$. If $A$ is chosen sufficiently small, then the local leaves $L_{a}$ and $L_{a}^{\prime}$ of the foliations $\mathcal{F}$ and $\mathcal{F}^{\prime}$ around $a \in A$ will satisfy the conditions that for $a_{1} \neq a_{2}$ the intersections $L_{a_{1}} \cap L_{a_{2}}$ and $L_{a_{1}}^{\prime} \cap L_{a_{2}}^{\prime}$ are empty, and so a map $\alpha:\left\{\bigcup L_{a} \mid a \in A\right\} \rightarrow\left\{\bigcup L_{a}^{\prime} \mid a \in A\right\}$ arises. Note that $U=\left\{\bigcup L_{a} \mid a \in A\right\}$ and $U^{\prime}=\left\{\cup L_{a}^{\prime} \mid a \in A\right\}$ are neighbourhoods of the stratum $e\left(S_{i}\right)$ in $P^{n+k} \times \mathbb{R}^{N}$. The map $\alpha$ will have differential equal to the identity at the points $a \in e\left(S_{i}\right)$. Hence $\alpha$ is a diffeomorphism in a neighbourhood of $e\left(S_{i}\right)$ and $\alpha(\mathcal{F})=\mathcal{F}^{\prime}$ around $e\left(S_{i}\right)$. Joining the points $x$ and $\alpha(x)$ by a minimal geodesic curve in the horizontal section they both belong to, we see that $\alpha$ can be embedded into an isotopy $\alpha_{t},(0 \leq t \leq 1)$ such that $\alpha_{0}=$ identity, $\alpha_{1}=\alpha$. Next we repeat the same procedure around $S_{i-1}$. Note that where the foliations $\mathcal{F}$ and $\mathcal{F}^{\prime}$ already coincide the above procedure gives automatically the identity diffeomorphism. And so on. In $i$ steps we obtain a diffeomorphism (moreover an isotopy) mapping $\mathcal{F}$ into $\mathcal{F}^{\prime}$ around $e\left(M^{n}\right)$.

\section{Cobordism of $\tau$-embeddings}

Definition 45 The cobordism of $\tau$-embeddings $\left(M^{n}, Q^{q}, e, V, \mathcal{F}\right)$ of $n$-manifolds in the manifold $Q^{q}$ can be defined in an obvious way. The cobordism is a $\tau$-embedding in $Q^{q} \times[0,1]$. Let us denote the set of equivalence classes by $\operatorname{Emb}_{(\tau, N)}\left(Q^{q}\right)$, where $N=q-n-k$.

The next theorem shows that the computation of the cobordism group of $\tau$-maps can be reduced to that of $\tau$-embeddings. 
Theorem 2 Let $P^{n+k}$ be a fixed oriented manifold of dimension $n+k$, where $k>0$ and consider the group $\operatorname{Cob}_{\tau}\left(P^{n+k}\right)$ of $\tau$-maps of oriented $n$-manifolds in $P^{n+k}$, where $\tau$ is a family of stable singularities, such that for each natural number $c$ there are finitely many elements in $\tau$ of codimension $c$. If $N$ is sufficiently big, then

$$
\operatorname{Cob}_{\tau}\left(P^{n+k}\right) \approx \operatorname{Emb}_{(\tau, N)}\left(P^{n+k} \times \mathbb{R}^{N}\right) .
$$

Proof 1) We define a map $\alpha: \operatorname{Cob}_{\tau}\left(P^{n+k}\right) \rightarrow \operatorname{Emb}_{(\tau, N)}\left(P^{n+k} \times \mathbb{R}^{N}\right)$ as follows: Let $f: M^{n} \rightarrow P^{n+k}$ be a $\tau$-map, and let $g: M^{n} \hookrightarrow \mathbb{R}^{N}$ be an arbitrary embedding. Put $e=f \times g$. Then $e: M^{n} \hookrightarrow P^{n+k} \times \mathbb{R}^{N}$ is an embedding and choosing for $\left\{v_{1}, \ldots, v_{N}\right\}$ the standard vertical vector fields (defined by the standard basis in $\mathbb{R}^{N}$ ) and for $\mathcal{F}$ the vertical foliations with leaves $\{p\} \times R^{N}, p \in P^{n+k}$, we obtain a $\tau-$ embedding $\left(M^{n}, P^{n+k} \times \mathbb{R}^{N}, e, V, \mathcal{F}\right)$, where $V=\left\{v_{1}, \ldots, v_{N}\right\}$. The cobordism class of this $\tau$-embedding does not depend on the choice of $g$. Indeed, if $g^{\prime}$ is another embedding $g^{\prime}: M^{n} \hookrightarrow \mathbb{R}^{N}$, then $g$ and $g^{\prime}$ can be joined by an isotopy $g_{t}, t \in[0,1]$, $g_{0}=g, g_{1}=g^{\prime}$ and putting $G(x, t)=\left(g_{t}(x), t\right) \in \mathbb{R}^{N} \times[0,1]$ for $x \in M^{n}, t \in[0,1]$ the $\tau$-embedding $E=f \times G: M^{n} \times[0,1] \rightarrow P^{n+k} \times \mathbb{R}^{N} \times[0,1]$, (where $V$ is chosen to be the vertical foliation for each $P^{n+k} \times \mathbb{R}^{N} \times\{t\}$ ), gives a cobordism joining $f \times g$ with $f \times g^{\prime}$.

Now let us choose any other representative $f^{\prime}$ of the same $\tau$-cobordism class $[f] \epsilon$ $\operatorname{Cob}_{\tau}\left(P^{n+k}\right)$. Then there exists an $(n+1)$-manifold $W^{n+1}$ such that $\partial W=-M^{n} \cup$ $M^{\prime}$, and a $\tau$-map

$$
F:\left(W ; M^{n} ; M^{\prime}\right) \rightarrow\left(P^{n+k} \times[0,1] ; P^{n+k} \times\{0\} ; P^{n+k} \times\{1\}\right) .
$$

such that $\left.F\right|_{M} ^{n}$ and $\left.F\right|_{M^{\prime}}$ coincide with $f$ and $f^{\prime}$ respectively. Further there is an embedding $G^{W}: W^{n+1} \hookrightarrow \mathbb{R}^{N}$ such that $\left.G^{W}\right|_{M^{n}}=g$ and $\left.G^{W}\right|_{M^{\prime}}=g^{\prime}$. Then $E^{W}=$ $F \times G^{W}: W^{n+1} \rightarrow P^{n+k} \times[0,1] \times \mathbb{R}^{N}$ is a $\tau$-embedding giving a cobordism between $e=f \times g$ and $e^{\prime}=f^{\prime} \times g^{\prime}$. Hence the map $\alpha: \operatorname{Cob}_{\tau}\left(P^{n+k}\right) \rightarrow \operatorname{Emb}_{(\tau, N)}\left(P^{n+k} \times \mathbb{R}^{N}\right)$ is well defined.

2) Next we define a map $\beta: \operatorname{Emb}_{(\tau, N)}\left(P^{n+k} \times \mathbb{R}^{N}\right) \rightarrow \operatorname{Cob}_{\tau}\left(P^{n+k}\right)$. Consider a $\tau$-embedding $\left(M^{n}, P^{n+k} \times \mathbb{R}^{N}, e, V, \mathcal{F}\right)$. By Theorem 1 and Lemma 43 the foliation $\mathcal{F}$ can be made vertical by an isotopy $\varphi_{t}, \varphi_{0}=$ identity, and $\varphi_{1} \circ e$ is a $(\tau, N)-$ embedding with vertical leaves. Projecting $\varphi_{1} \circ e: M^{n} \hookrightarrow P^{n+k} \times \mathbb{R}^{N}$ to $P^{n+k}$ we obtain a $\tau$-map $f: M^{n} \rightarrow P^{n+k}$. We have to show that

a) the cobordism class of $[f]$ is independent of the choice of the isotopy $\varphi_{t}$, and

b) if $e^{\prime}$ is another $\tau$-embedding cobordant to $e$, then applying the above procedure to $e^{\prime}$ we obtain a $\tau$-map $f^{\prime}$ which is $\tau$-cobordant to $f$. 
Both of these statements follow from the relative version of the Stratified Compression Theorem. First we prove $\mathrm{b}$ ). Suppose that $e$ and $e^{\prime}$ are cobordant $\tau$-embeddings joined by a $\tau$-embedding $E$. Apply isotopies $\varphi_{t}$ and $\varphi_{t}^{\prime}$ turning vertical the leaves of the foliations of the $\tau$-embeddings $e$ and $e^{\prime}$ respectively. $\varphi_{t}$ and $\varphi_{t}^{\prime}$ are defined on $P^{n+k} \times \mathbb{R}^{N} \times\{0\}$ and $P^{n+k} \times \mathbb{R}^{N} \times\{1\}$, respectively. They can be extended to an isotopy $\Phi_{t}$ of the whole cylinder $P^{n+k} \times \mathbb{R}^{N} \times[0,1]$. As a result we obtain a $\tau-$ embedding $\Phi_{1} \circ E$ with boundary such that its foliation is vertical on the boundary. Now applying the relative version of Theorem 1 and Lemma 43 we obtain a $\tau$-embedding cobordism in $P^{n+k} \times \mathbb{R}^{N} \times[0,1]$ with vertical leaves everywhere. Projecting it to $P^{n+k} \times[0,1]$ we obtain a $\tau$-cobordism between the $\tau$-maps $\pi \circ \varphi_{1}^{\prime} \circ e^{\prime}$ and $\pi \circ \varphi_{1} \circ e$, where $\pi: P^{n+k} \times \mathbb{R}^{N} \rightarrow P^{n+k}$ is the projection. That proves b). In order to prove a) it is enough to put $e^{\prime}=e$ in the previous argument.

Remark 46 The same proof shows the following:

1) If $N$ is sufficiently big (compared with $n, k$ and $\ell$ ), then

$$
\operatorname{Cob}_{\tau \oplus \ell}\left(P^{n+k}\right) \approx \operatorname{Emb}_{(\tau, N+\ell)}\left(P^{n+k} \times \mathbb{R}^{N}\right) .
$$

2) $\operatorname{Emb}_{(\tau, N)}\left(P^{n+k} \times \mathbb{R}^{N}\right)$ does not depend on $N$, moreover the embedding $\mathbb{R}^{N} \subset$ $\mathbb{R}^{N+1}$ induces an isomorphism

$$
\operatorname{Emb}_{(\tau, N)}\left(P^{n+k} \times \mathbb{R}^{N}\right) \approx \operatorname{Emb}_{(\tau, N+1)}\left(P^{n+k} \times \mathbb{R}^{N+1}\right) .
$$

Definition 47 For any natural numbers $n, N, k$, any manifold $Q^{n+k+N}$ and set of stable singularities $\tau$ of codimension $k$ maps put

$$
\operatorname{Emb}_{(\tau, N)}^{\text {stable }}\left(Q^{n+k+N}\right) \stackrel{\text { def }}{=} \lim _{L \rightarrow \infty} \operatorname{Emb}_{(\tau, N+L)}\left(Q^{n+k+N} \times \mathbb{R}^{L}\right) .
$$

Our conclusion from this section is that in order to prove the Pontrjagin-Thom construction for $\tau$-maps it is enough to do that for $(\tau, N)$-embeddings with big $N$. The key tool for the latter will be the normal form of a $\tau$-embeddings.

\section{Normal forms of $\tau$-embeddings of bounded source dimen- sions}

\subsection{Normal form around a stratum}

Fix a natural number $n$. In this subsection we shall consider $(\tau, N)$-embeddings with source manifolds of dimensions less or equal to $n$. Let $\eta$ be a top singularity 
stratum in $\tau$. Choose a finite dimensional approximation $B G_{\eta \text {,fin }}$ of $B G_{\eta}$ such that the pair $\left(B G_{\eta}, B G_{\eta, \text { fin }}\right)$ is $(n+1)$-connected. (Hence for the purpose of classification of $G_{\eta}$-bundles with base space of dimensions less or equal to $n$ the approximation $B G_{\eta \text {,fin }}$ is fine enough.) It can be supposed that $B G_{\eta \text {,fin }}$ is a smooth manifold, let us denote its dimension by $\mu(n)$.

Let us denote by $\xi_{\text {fin }}$ and $\widetilde{\xi}_{\text {fin }}$ the restrictions of the bundles $\xi_{\eta} \rightarrow B G_{\eta}$ and $\tilde{\xi}_{\eta} \rightarrow B G_{\eta}$ to $B G_{\eta \text {,fin }}$, the corresponding restriction of the map $\Phi_{\eta}: \xi_{\eta} \rightarrow \widetilde{\xi}_{\eta}$ will be denoted by $\Phi_{\eta, \text { fin }}: \xi_{\eta, \text { fin }} \rightarrow \widetilde{\xi}_{\eta, \text { fin }}$.

Put $N(n)=2 \mu(n)+2 n+2$. Let $N$ be any number such that $N \geq N(n)$, and let $j_{\eta}: \xi_{\eta, \text { fin }} \rightarrow \mathbb{R}^{N}$ be a smooth embedding. (The number $N(n)$ was chosen so that for $N \geq N(n)$ such an embedding exists and it is unique up to isotopy.) Put $\Phi_{\eta, \text { fin }}^{\text {lift }}=\left(\Phi_{\eta, \text { fin }}, j_{\eta}\right): \xi_{\text {fin }} \rightarrow \widetilde{\xi}_{\eta, \text { fin }} \times \mathbb{R}^{N}$.

This map $\Phi_{\eta \text {,fin }}^{\text {lift }}$ is a $(\tau, N)$-embedding. (The foliation is the vertical one with leaves parallel to the Euclidean factor.)

Definition 48 The map $\Phi_{\eta \text {,fin }}^{\text {lift }}$ will be called the normal form of $(\tau, N)$-embeddings around the stratum $\eta$ and in dimension $\leq n$.

The reason for this name is the following:

Theorem 3 Let $e: M^{n} \hookrightarrow Q^{q}$ be a $(\tau, N)$-embedding, with $N \geq N(n)$. Let $\eta(e)$ be the stratum of $\eta$-points of $e$, and $\tilde{\eta}(e)=e(\eta(e))$ its image. Then there exist tubular neighbourhoods $T$ and $\widetilde{T}$ of $\eta(e)$ and $\widetilde{\eta}(e)$ respectively, such that $T=e^{-1}(\widetilde{T})$ and the restriction map $\left.e\right|_{T}: T \rightarrow \widetilde{T}$ can be induced from the map $\Phi_{\eta, \text { fin }}^{\text {lift }}$, ie there is a commutative diagram:

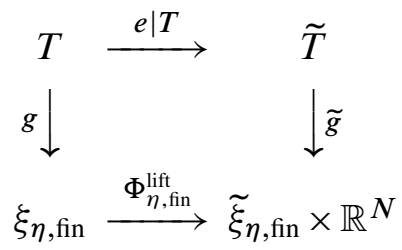

Here $g$ and $\tilde{g}$ are fibrewise maps, isomorphic on each fibre.

Definition 49 Given two smooth disc bundles $T$ and $\widetilde{T}$ over the same smooth manifold (possibly with boundary) and a fibrewise map $\mathcal{E}: T \rightarrow \widetilde{T}$, we shall say that this map is provided with a $G_{\eta} \times \mathrm{id}_{\mathbb{R}^{N}}$-structure, if fibrewise maps $g$ and $\tilde{g}$ are given, such that diagram (2) holds (substituting $\mathcal{E}$ for $e \mid T$ ), the maps $g$ and tildeg map fibres dippeomorphically onto fibres. Note that a $G_{\eta} \times \mathrm{id}_{\mathbb{R}^{N}}$-structure defines on the target bundle $\tilde{T}$ an $N$-dimensional foliation. 
Remark 50 The fibrewise homotopy classes of the maps $g$ and $\widetilde{g}$ are unique.

Theorem 4 (Relative version of the normal form around a stratum) Suppose that the maps $g$ and $\tilde{g}$ are given over a subset $A \subset \eta(e)$ and they satisfy the commutativity expressed by diagram (2). Then they can be extended to the maps $g$ and $\tilde{g}$.

Proof Theorem 3 and Theorem 4 follow immediately from Proposition 28. Nevertheless we give details in Section 9.3.

\subsection{Global normal form of $(\tau, N)$-embeddings}

Let $\tau$ be a finite sequence of singularities $\tau=\left\{\left[\eta_{0}\right]<\left[\eta_{1}\right] \cdots<\left[\eta_{r}\right]\right\}$, and let $n$ be any natural number. We shall simplify the notation by writing $\xi_{i}, \xi_{i, \text { fin }}, \widetilde{\xi}_{i}, \widetilde{\xi}_{i \text {,fin }}$ and $j_{i}$ instead of $\xi_{\eta_{i}}, \xi_{\eta_{i}, \text { fin }}, \widetilde{\xi}_{\eta_{i}}, \widetilde{\xi}_{\eta_{i}}$, fin and $j_{\eta_{i}}$ respectively. Recall these objects:

- $\xi_{i}$ is the universal normal bundle in the source manifold of the stratum $\eta_{i}$.

- $\xi_{i, \text { fin }}$ is its final dimensional (manifold) approximation) ("good enough" for $n$-dimensional base spaces).

- $\tilde{\xi}_{i}$ is the universal normal bundle of the $\eta_{i}$-stratum in the target.

- $\widetilde{\xi}_{i, \text { fin }}$ is its finite dimensional approximation.

- $j_{i}$ is the embedding of $\xi_{i \text {,fin }}$ into $R^{N}$, unique up to isotopy.

Proposition 51 There is a natural number $N(n)$ depending on $n$, such that for any $N \geq N(n)$ a $(\tau, N)$-embedding $\left(M^{n}, e, Q^{q}, V, \mathcal{F}\right),(N=q-n-k)$ has the following structure:

There are subsets $T_{i}$, for $i=1,2, \ldots, r$ in $M^{n}$ and subsets $\widetilde{T}_{i}$, for $i=1,2, \ldots, r$ in $Q$ such that:

a) $M^{n}=T_{r} \cup T_{r-1} \cup \cdots \cup T_{0}$.

b) $Q \supset \widetilde{T}=\widetilde{T}_{r} \cup \widetilde{T}_{r-1} \cdots \cup \widetilde{T}_{0}$, where $\widetilde{T}$ is a tubular neighbourhood of $e\left(M^{n}\right)$ in $Q$.

c) $\tilde{T}_{i}$ is an open set in $Q \backslash \bigcup_{j>i} \widetilde{T}_{j}$, it is a tubular neighbourhood of $\tilde{\eta}_{i}(e) \backslash$ $\bigcup_{j>i} \tilde{T}_{j}$, where the sets $T_{r+1}$ and $\widetilde{T}_{r+1}$ are empty.

d) $T_{i}=e^{-1}\left(\left(\widetilde{T}_{i}\right), T_{i}\right.$ is an open set in $M^{n} \backslash \bigcup_{j>i} T_{j}$, it is a tubular neighbourhood of $\eta_{i}(e) \backslash \bigcup_{j>i} T_{j}$,.

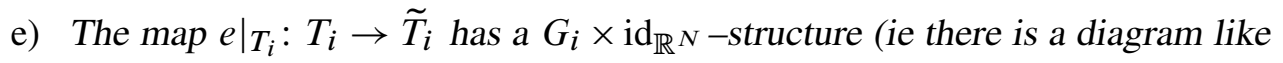
(2).) 
f) (Compatibility condition) There exist $T_{i}^{\prime}$ and $\widetilde{T}_{i}^{\prime}$ slightly bigger neighbourhoods of $T_{i}$ and $\widetilde{T}_{i}$ for $i=1,2, \ldots, r$ such that $e^{-1}\left(\widetilde{T}_{i}^{\prime}\right)=T_{i}^{\prime}$ and the restriction

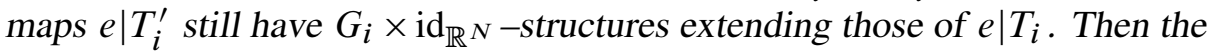
leaves of the foliations on the overlapping parts of the enlarged neighbourhoods $\tilde{T}_{i}^{\prime}$ coincide for all indices $i$.

Proof Backward induction on the indices of the strata. Apply first Theorem 3 to the map $T_{r}^{\prime} \rightarrow \widetilde{T}_{r}^{\prime}$, and then apply the relative version Theorem 4 to

$T_{i}^{\prime} \backslash \bigcup_{j>i} T_{j} \rightarrow \tilde{T}_{i}^{\prime} \backslash \bigcup_{j>i} \tilde{T}_{j}$ modulo the map $T_{i}^{\prime} \cap\left(\bigcup_{j>i} T_{j}^{\prime}\right) \rightarrow \tilde{T}_{i}^{\prime} \cap\left(\bigcup_{j>i} \tilde{T}_{j}^{\prime}\right)$.

\subsection{Pulling back a $(\tau, N)$-embedding}

The following is an extension of Thom's lemmas (see Theorem I.5 and Lemma IV. 5' of [43]); its proof is omitted.

\section{Lemma 52}

a) Let $f: V^{n} \rightarrow M^{p}$ be a $C^{\infty}$ smooth map, $N^{p-m}$ a compact submanifold in $M^{p}$, let $T_{N}$ be a tubular neighbourhood of $N^{p-m}$. Suppose that $f$ is transverse to $N^{p-m}$, and hence the preimage $L^{n-p}=f^{-1}\left(N^{p-m}\right)$ is a submanifold in $V^{n}$, let us denote its tubular neighbourhood by $T_{L}$. We can suppose that $T_{N}$ and $T_{L}$ are chosen so that $T_{L}=f^{-1}\left(T_{N}\right)$. Note that $T_{L}$ and $T_{N}$ are ball bundles over $L$ and $N$ respectively with fibres $D^{m}$. Let $\pi_{L}: T_{L} \rightarrow L$ and $\pi_{N}: T_{N} \rightarrow N$ be the fibreing maps. Let $D^{\prime m} \subset D^{m}$ be a smaller (concentric) ball in $D^{m}$ and let $\pi_{L}^{\prime}: T_{L}^{\prime} \rightarrow L$ and $\pi_{N}^{\prime}: T_{N}^{\prime} \rightarrow N$ be the smaller tubular neighbourhoods and associated fibre maps with fibre $D^{\prime m}$.

Then there is a diffeomorphism $A$ of $T_{N}$ onto itself, isotopic to the identity and fixed near the boundary of $T_{N}$ such that the map $A \circ f$ maps the fibres of $\pi_{L}^{\prime}$ onto those of $\pi_{N}^{\prime}$ diffeomorphically.

b) (Relative version) Let $K$ be a compact subset of $N$, and $U$ its open neighbourhood in $N$. Then if the isotopy $A$ is given on $\pi^{-1}(U)$ then it can be chosen so for the whole tubular neighbourhood $T_{N}$ that it coincide with the given one on $\pi^{-1}\left(U^{\prime}\right)$, where $U^{\prime}$ is a neighbourhood of $K$ (possibly smaller than $U$ ).

Now let $\left(M^{n}, Q^{q}, e, V, \mathcal{F}\right)$ be a $(\tau, N)$-embedding, $(N=q-n-k)$. Let $\widetilde{T}_{i}^{\prime}$ be the slightly enlarged sets $\widetilde{T}_{i}$ in $Q^{q}$, described in the global normal form of a $(\tau, N)-$ embedding and corresponding to the $(\tau, N)$-embedding $e$.

Let $Q^{\prime}$ be a manifold of dimension $q^{\prime}$ and let $\varphi: Q^{\prime} \rightarrow Q^{q}$ be a map transverse to all the strata of $e$. Apply the relative version of Lemma 52 by a backward induction to 
the sets $\widetilde{T}_{i}$, starting with $i=r$ and keeping the isotopy constructed in the $i$-th step fixed on the set $\widetilde{T}_{i}^{\prime} \backslash \widetilde{T}_{i}$ during the $(i-1)$-th step. Finally we obtain an isotopy $A$ on $Q^{q}$ such that the map $\varphi^{*}=A \circ \varphi$ maps diffeomorphically the fibres of the tubular neighbourhoods of the preimages of the strata to those of the tubular neighbourhoods $\widetilde{T}_{i}$.

Now we obtain a $(\tau, N)$-embedding $\left(M^{\prime}, Q^{\prime}, e^{\prime}, V^{\prime}, \mathcal{F}^{\prime}\right)$ just taking the preimages of the corresponding objects in $\left(M^{n}, Q^{q}, e, V, \mathcal{F}\right)$. In particular the leaves of the foliation $\mathcal{F}$ are the preimages of those of $\mathcal{F}$ under $\varphi^{*}$. This does make sense because $\varphi^{*}$ maps the fibres of the tubular neighbourhoods diffeomorphically. This fact implies also that the local projection along the leaves of the manifold $M^{\prime}=\left(\varphi^{*}\right)^{-1}\left(e\left(M^{n}\right)\right)$ will have only singularities from $\tau$. The cobordism class of the obtained pulled back $(\tau, N)-$ embedding $\left(M^{\prime}, Q^{\prime}, e^{\prime}, V^{\prime}, \mathcal{F}^{\prime}\right)$ in $\operatorname{Emb}_{(\tau, N)}\left(Q^{\prime}\right)$ does not depend on the choices made during the construction. We can summarise the pulling back procedure as follows.

Proposition $53 \tau$-embeddings have the following functor-like property. Let $\varphi: Q^{\prime} \rightarrow$ $Q^{q}$ be a smooth map, transverse to all the strata of a given $(\tau, N)$-embedding $\left(M^{n}, e, Q^{q}, V, \mathcal{F}\right)$. Then $\varphi^{-1}\left(M^{n}\right) \subset Q^{\prime}$ will be a $(\tau, N)$-embedding with respect to the foliation $\mathcal{F}^{\prime}=\varphi^{-1} \circ A^{-1}(\mathcal{F})$, where $A$ is the diffeomorphism of $Q^{q}$ described in the application of Lemma 52 to pulling back a $(\tau, N)$-embedding.

Definition 54 Let $e: M^{n} \hookrightarrow Q$ be a $\tau$-embedding and $\varphi: Q^{\prime} \rightarrow Q$ be any map. Let $\varphi^{\prime}$ be any map homotopic to $\varphi$ and transverse to all the strata of $e\left(M^{n}\right)$. The cobordism class of $\tau$-embeddings of the pulled back $\tau$-embedding $\left(\varphi^{\prime}\right)^{-1}\left(M^{n}\right)$ is called the cobordism class associated to the map $\varphi$.

Remark 55 Clearly homotopic maps $\varphi_{0}: Q^{\prime} \rightarrow Q$ and $\varphi_{1}: Q^{\prime} \rightarrow Q$ associate to a given $(\tau, N)$-embedding $e: M^{n} \hookrightarrow Q$ the same cobordism class in $\operatorname{Emb}_{(\tau, N)}\left(Q^{\prime}\right)$.

Indeed, approximations of the maps $\varphi_{0}$ and $\varphi_{1}$ transverse to the strata can be joined by a homotopy transverse to the strata. Now the preimage of $e\left(M^{n}\right)$ under the homotopy will provide a cobordism of $\tau$-embeddings. Similarly it can be shown, that the associated cobordism class will be the same for two $\tau$-embeddings that are cobordant. Hence a map arises

$$
\left[Q^{\prime}, Q^{q}\right] \times \operatorname{Emb}_{(\tau, N)}\left(Q^{q}\right) \rightarrow \operatorname{Emb}_{(\tau, N)}\left(Q^{\prime}\right)
$$

where $\operatorname{dim} Q^{\prime}-\operatorname{dim} Q^{q}=n^{\prime}-n$, and $\left[Q^{\prime}, Q^{q}\right]$ denotes the set of homotopy classes of maps, and $n^{\prime}$ denotes the dimension of the source manifold of the $(\tau, N)$ embedding into $Q^{\prime}$. 


\section{Pontrjagin-Thom construction for $\tau$-embeddings}

Here we shall show that for any $q$-dimensional manifold $Q^{q}$ the group $\operatorname{Emb}_{(\tau, N)}^{\text {stable }}\left(Q^{q}\right)$ admits a homotopical description: it is isomorphic to the group of stable homotopy classes of maps $\left\{Q^{q}, S^{N} V_{\tau}\right\}$ from $Q$ to the $N$-th suspension of a certain (virtual) space $V_{\tau}$. (Here $N=q-n-k$.) The problem is that we can construct the "classifying space" $V_{\tau}$ only in some stable sense. For giving the precise meaning of this last sentence we introduce the notion of the virtual complex.

\subsection{Virtual complexes}

Virtual complexes will be - roughly speaking - CW complexes in which the gluing maps of the cells are given only as stable maps. In our case this notion will arise as a result of a gluing procedure using stable maps. So we give first the definition of such a gluing procedure using usual, ie nonstable maps. (So the next definition is well known, we give it only to make clear the analogy and the differences with the definition following it.)

Definition 56 Suppose that a sequence of $\mathrm{CW}$ pairs $\left(A_{j}, B_{j}\right), j=0,1,2, \ldots$ is given and further attaching maps $\rho_{j}$ are defined on $B_{j}$ with values in inductively constructed spaces $X_{j-1}$ (see below), then we can obtain a limit space $X=\lim _{j \rightarrow \infty} X_{j}$ by the following well-known gluing procedure:

0) $X_{0} \stackrel{\text { def }}{=} A_{0}$. Let a map $\varrho_{1}: B_{1} \rightarrow X_{0}$ be given.

1) $X_{1} \stackrel{\text { def }}{=} A_{1} \cup_{\varrho_{1}} X_{0}=A_{1} \sqcup X_{0} / b \sim \varrho_{1}(b), \forall b \in B_{1}$. ie in the disjoint union $A_{1} \sqcup X_{0}$ we identify each point $b \in B_{1}$ with its image $\rho_{1}(b) \in X_{0}$.

Let a map $\varrho_{2}: B_{2} \rightarrow X_{1}$ be given.

2) $X_{2} \stackrel{\text { def }}{=} A_{2} \cup_{\varrho_{2}} X_{1}=A_{2} \sqcup X_{1} / b \sim \varrho_{2}(b), \forall b \in B_{2}$. Let a map $\varrho_{3}: B_{3} \rightarrow X_{2}$ be given, etc.

Let us denote by $c_{j}$ the connectivity of the pair $\left(A_{j}, B_{j}\right)$, and suppose that the sequence $c_{j}$ goes to infinity as $j \rightarrow \infty$. For simplicity we suppose that this sequence $\left(c_{j}\right)$ of connectivities is monotonic. Put $X=\lim _{\longrightarrow} X_{j}$.

Clearly the $n$-homotopy type of $X$ is well defined for any $n$.

Example 57 Any CW complex having in each dimension finitely many cells is obtained in this way if for each $j=1,2, \ldots$ we take $\left(A_{j}, B_{j}\right)=\left(D^{c_{j}+1}, S^{c_{j}}\right)$ a closed ball of dimension $c_{j}+1$ and its boundary. 
Definition 58 (Virtual complex) Suppose that we are almost in the same situation as before with the only difference that the attaching maps $\rho_{j}$ are given only in stable sense. More precisely:

0) A sequence of CW pairs $\left(A_{j}, B_{j}\right), j=0,1,2, \ldots$ is given. Put $X_{0}=A_{0}$.

1) A stable map $\varrho_{1}: B_{1} \nrightarrow \nrightarrow X_{0}$ is given ${ }^{7}$, ie there exists $n_{1}$ such that a map $S^{n_{1}} B_{1} \rightarrow S^{n_{1}} X_{0}$ is given. We shall denote this map by $S^{n_{1}} \varrho_{1}$, although $\varrho_{1}$ may not exist. Put

$$
S^{n_{1}} X_{1} \stackrel{\text { def }}{=} S^{n_{1}} A_{1} \sqcup S^{n_{1}} X_{0} / b \sim S^{n_{1}} \varrho_{1}(b)
$$

$\forall b \in S^{n_{1}} B_{1} \subset S^{n_{1}} A_{1}$. Note, that $X_{1}$ may not exist.

2) A stable map $\varrho_{2}: B_{2} \not \supset X_{1}$ is given, ie there exist $n_{2}>n_{1}$ such that a map $S^{n_{2}} \varrho_{2}: S^{n_{2}} B_{2} \rightarrow S^{n_{2}} X_{1}\left(=S^{n_{2}-n_{1}}\left(S^{n_{1}} X_{1}\right)\right)$ is given. We shall denote this map by $S^{n_{2}} \varrho_{2}$, although $\varrho_{2}$ may not exist. Put

$$
S^{n_{2}} X_{2} \stackrel{\text { def }}{=} S^{n_{2}} A_{2} \sqcup S^{n_{2}} X_{1} / b \sim S^{n_{2}} \varrho_{2}(b)
$$

$\forall b \in S^{n_{2}} B_{2} \subset S^{n_{2}} A_{2}$. Note again, that $X_{2}$ may not exist.

3) And so on:

$$
S^{n_{i}} X_{i} \stackrel{\text { def }}{=} S^{n_{i}} A_{i} \sqcup S^{n_{i}} X_{i-1} / b \sim S^{n_{i}} \varrho_{i}(b)
$$

Here $n_{i}>n_{i-1}$.

Again suppose that the connectivity $c_{j}$ of $\left(A_{j}, B_{j}\right)$ tends to infinity monotonically. Does the limit space $X=\lim X_{j}$ make sense even if the spaces $X_{j}$ may not exist? We show that $X$ exists in the following stable sense.

For each $m$ there is a natural number $N(m)$ such that for $N>N(m)$ the $(N+m)-$ homotopy type of the space $S^{N} X$ is well defined. Indeed, let $i$ be such that $c_{i}>m$, and let $N(m)$ be $n_{c_{i}}$. Then the $S^{N}$ suspensions exist for all the virtual spaces $X_{0}, X_{1}, \ldots, X_{i}$ and the maps $S^{N} \varrho_{i}$ exist for $j=0,1, \ldots, i$. We claim that the $(N+m)$-th homotopy type of $S^{N} X_{i}$ can be taken for that of $S^{N} X$. Indeed, if $i^{\prime}>i$, and $N^{\prime}>N$ is such that the suspension $S^{N^{\prime}} X_{j}$ exists for each $j=0,1, \ldots, i^{\prime}$, then the $\left(N^{\prime}+m\right)$-th homotopy type of $S^{N^{\prime}} X_{i^{\prime}}$ coincides with the $\left(N^{\prime}-N\right)$-th suspension of the $(N+m)$-th homotopy type of $S^{N} X_{i}$.

We will say that $X$ exists as a virtual complex, and will denote it by $X=\dot{\cup}_{\varrho_{j}} A_{j}$. The dot will indicate that the union is taken in virtual (ie stable) sense.

\footnotetext{
${ }^{7}$ The sign $\nrightarrow \rightarrow$ means "stable map".
} 
Remark 59 For a virtual complex $X$ its de-suspension can be defined as follows: It is a virtual complex $Y=\lim Y_{j}$, where $S^{n_{j}+1} Y_{j}=S^{n_{j}} X_{j}$. (Note the $Y_{j}$ may not exist.)

Remark 60 Note that $\Gamma X=\Omega^{\infty} S^{\infty} X$ is a space well-defined up to homotopy if $X$ is a virtual complex. Indeed we say that the $m$-homotopy type of $\Gamma X$ is that of $\Omega^{N} S^{N} X_{i}$, where $N$ and $i$ are as above.

Definition 61 There is an obvious modification of the above definition when the maps $S^{n_{j}} B_{j} \rightarrow S^{n_{j}} X_{j-1}$ are defined only on the ( $n_{j}$-suspension of the) $m_{j}$ skeleton of $B_{j}$, where $m_{j} \geq m_{j-1}, m_{j} \rightarrow \infty$ as $j \rightarrow \infty$. Then the space $\Gamma X$ still can be defined:

$$
\Gamma X=\lim _{j \rightarrow \infty} \Omega^{n_{j}} S^{n_{j}} \mathrm{sk}_{m_{j}} X_{j} .
$$

Remark 62 It is a natural feeling of the reader that virtual complex is just another name for the notion of the spectrum. In the present Remark we discuss the relation of these two notions. In order to obtain a spectrum from a virtual complex one has to choose finite dimensional approximations of the blocks $\left(A_{i}\right)$ attached stably to the previously obtained spaces $\left(X_{i-1}\right)$. Two different choices of the sequence of approximations give different spectra, but they are equivalent in a natural sense, in particular they define the same extraordinary cohomology theories. So the advantage of the notion of the virtual complex is that one does not have to choose data (the sequence of approximations) that finally turn out to be unimportant. On the other hand a virtual complex has a filtration, an essential one, that reflects the steps of its construction ( $X_{i}$ in the definition). So one can say, that a virtual complex defines an equivalence class of spectra without pointing out a particular spectrum of this equivalence class.

\subsection{Construction of the virtual complex $V_{\tau}$}

Recall that $\tau=\left\{\left[\eta_{0}\right]<\left[\eta_{1}\right]<\cdots<\left[\eta_{i}\right]\right\}$, where each $\left[\eta_{j}\right]$ is a stable, codimension $k$ map-germ class for $j=0, \ldots, i$. They are ordered according to their hierarchy, (more precisely in a way compatible with the partial ordering given by their hierarchy). Further let the set $\tau$ be "closed" in the sense that $[\xi] \in \tau$ and $[\zeta]<[\xi]$ imply $\zeta \in \tau$. (This assumption implies that $\left[\eta_{0}\right]$ is the class of germs of maximal rank.) In Section 2 we introduced for $\eta=\eta_{j}$ the notation: $G_{j}, \xi_{j}, \widetilde{\xi}_{j}$, for the objects $G_{\eta_{j}}, \xi_{\eta_{j}}, \widetilde{\xi}_{\eta_{j}}$. For $\tau_{j}=\left\{\eta_{0}<\cdots<\eta_{j}\right\}, j \leq i$, we denoted the space $\mathcal{K}_{\tau_{j}}$ simply by $\mathcal{K}_{j}$. Recall that $\mathcal{K}_{i}=\bigcup\left\{D\left(\xi_{j}\right) \mid j \leq i\right\}$ for an appropriate gluing.

In this subsection we shall show that the target disc bundles $D\left(\widetilde{\xi}_{j}\right)$ can be attached to each other in "virtual sense" and give a virtual complex $V_{\tau}$. More precisely we will attach finite dimensional approximations of these disc bundles, (see Definition 61). 
For a noncompact space $Q$ we denote by $\dot{Q}$ the one point compactification. We put $\dot{Q}=Q$ if $Q$ is compact.

Theorem 5 (Construction of the virtual complex $V_{\tau}$ and the Pontrjagin-Thom construction for $\tau$-embeddings) There is a virtual complex

$$
V_{\tau}=\bigcup^{\bullet}\left\{D\left(\tilde{\xi}_{\eta}\right) \mid \eta \in \tau\right\}
$$

and for each natural number $n$ there is a natural number $N(n)$ such that, for $N \geq N(n)$ and for any manifold $Q$ of dimension $N+n+k$ the following statement holds:

$$
\operatorname{Emb}_{(\tau, N)}(Q) \approx\left\{\dot{Q}, S^{N} V_{\tau}\right\}
$$

Here the brackets \{ \} denote stable homotopy classes, ie

$$
\{X, Y\}=\lim _{N \rightarrow \infty}\left[S^{N} X, S^{N} Y\right] .
$$

Note that the stable homotopy classes $\{X, Y\}$ make sense also if $X$ is a space and $Y$ is a virtual complex.

Addendum 63 The isomorphism holds also if $Q$ is a manifold with corners or if it is a finite simplicial complex.

Addendum 64 (Naturality properties) For any manifold $Q$ (possibly with corners) we shall construct two homomorphisms $\alpha \operatorname{Emb}_{(\tau, N)}(Q) \rightarrow\left\{\dot{Q}, S^{N} V_{\tau}\right\}$ and $\beta:\left\{\dot{Q}, S^{N} V_{\tau}\right\} \rightarrow \operatorname{Emb}_{(\tau, N)}(Q)$ that are each others' inverses. The isomorphisms $\alpha$ and $\beta$ commute with the homomorphisms $f^{*}$ induced by a map $f:\left(Q^{\prime}, \partial Q^{\prime}\right) \rightarrow$ $(Q, \partial Q): f$ induces a map $f^{*}: \operatorname{Emb}_{(\tau, N)}(Q) \longrightarrow \operatorname{Emb}_{(\tau, N)}\left(Q^{\prime}\right)$ by pullback and a map $f^{*}:\left\{\dot{Q}, S^{N} V_{\tau}\right\} \longrightarrow\left\{\dot{Q}^{\prime}, S^{N} V_{\tau}\right\}$ by composition.

The isomorphisms $\alpha$ and $\beta$ will be constructed by an induction on $\tau$ together with the virtual complex $V_{\tau}$.

Definition 65 (of $V_{\tau}$ and the homomorphism $\alpha$ ) Given a natural number $n$ let $B G_{\eta, \text { fin }}$ be a finite dimensional approximation of $B G_{\eta}$ so that the pair $\left(B G_{\eta}, B G_{\eta, \text { fin }}\right)$ is $(n+1)$-connected. Let $\mu(n)$ be the dimension of $B G_{\eta, \text { fin }}$. Let $V_{\tau^{\prime} \text {,fin }}$ be such a finite dimensional approximation of $V_{\tau}^{\prime}$ that for some $N \geq 2 \mu(n)+2 n+2$ the suspension $S^{N} V_{\tau^{\prime}}$ exists and the pair $\left(V_{\tau^{\prime}}, V_{\tau^{\prime}, \text { fin }}\right)$ is $\mu(n)+n+1$-connected. Then $S^{N} V_{\tau^{\prime} \text {,fin }}$ classifies $\left(\tau^{\prime}, N\right)$-embeddings with source dimension not greater than $\mu(n)+n$. The map

$$
\Phi_{\eta, \text { fin }}^{\text {lift }}: \xi_{\eta, \text { fin }} \rightarrow \widetilde{\xi}_{\eta, \text { fin }} \times R^{N}
$$


is a $(\tau, N)$-embedding and let us denote by $\Phi_{S \text {,fin }}^{\text {lift }}$ or shortly by $\Phi_{S}$ the associated map of spherical bundles. More precisely we consider a small spherical bundle $S\left(\widetilde{\xi}_{\eta \text {,fin }}\right)$ of the vector bundle $\widetilde{\xi}_{\eta \text {,fin }}$ and its transverse preimage by $\Phi_{\eta \text {,fin }}$, and denote this preimage by $\mathcal{S}\left(\xi_{\eta \text {,fin }}\right)$. The mapping $\Phi_{\eta \text {,fin }}^{\text {lift }}$ maps $\mathcal{S}\left(\xi_{\eta \text {,fin }}\right)$ into $S\left(\widetilde{\xi}_{\eta \text {,fin }}\right) \times R^{N}$. This is the map that we shall denote by $\Phi_{S}$ and call the associated sphere bundle map.

$\Phi_{S}$ is a $\left(\tau^{\prime}, N\right)$-embedding with source dimension not greater than $\mu(n)+n$. Let us denote by $\left[\Phi_{S \text {,fin }}^{\text {lift }}\right]$ the cobordism class of this $\tau^{\prime}$-embedding. By the inductional assumption a map $\alpha\left(\left[\Phi_{S \text {,fin }}^{\text {lift }}\right]\right): S\left(\widetilde{\xi}_{\text {fin }} \times D^{N}\right) \rightarrow S^{N} V_{\tau^{\prime} \text {,fin }}$ arises. $\left(D^{N}\right.$ is a ball of sufficiently big radius, so that the image of $\Phi_{S}$ is contained in $S\left(\widetilde{\xi}_{\text {fin }} \times D^{N}\right)$.) Now put

$$
S^{N} V_{\tau, \text { fin }} \stackrel{\text { def }}{=} S^{N} V_{\tau^{\prime}, \text { fin }} \bigcup_{\varrho_{\text {fin }}} D\left(\widetilde{\xi}_{\text {fin }}\right) \times D^{N}
$$

where the attaching map $\varrho_{\text {fin }}$ is the map $\alpha\left(\left[\Phi_{S \text {,fin }}^{\text {lift }}\right]\right)$. This will be a finite-dimensional approximation of the $N$-th suspension of the virtual space $V_{\tau}$. Taking higher and higher dimensional approximations of $B G_{\eta}$ and of the bundles $\xi_{\eta}$ and $\widetilde{\xi}_{\eta}$ we obtain better and better approximations of the virtual complex $V_{\tau}$.

Remark 66 The attaching map $\rho_{\text {fin }}$ can be chosen so that the subsets

$$
\Phi_{\eta_{j}, \text { fin }}^{\mathrm{lift}}\left(D \xi_{j, \text { fin }}\right) \subset D \widetilde{\xi}_{j, \text { fin }} \times D^{N}
$$

for $j=1,2, \ldots, i$ will be attached to each other and they will form a finite dimensional approximation of the Kazarian space $\mathcal{K}_{\tau}=\bigcup D \xi_{j}$, (constructed by the gluing procedure). The obtained finite dimensional approximation will be denoted by $\mathcal{K}_{\tau, \text { fin }}=\bigcup D \xi_{i \text {,fin }}$. In the process of the proof we will construct the map $\rho_{\text {fin }}$ explicitly. It will also follow that the inclusion $\mathcal{K}_{\tau \text {,fin }} \subset S^{N} V_{\tau \text {,fin }}$ restricted to the bundle $D \xi_{i, \text { fin }}$ coincides with the map $\Phi_{\eta \text {,fin }}^{\text {lift }}: D \xi_{i, \text { fin }} \rightarrow D \widetilde{\xi}_{i, \text { fin }} \times \mathbb{R}^{N}$, composed with the map $D \widetilde{\xi}_{i, \text { fin }} \times \mathbb{R}^{N} \rightarrow S^{N} V_{\tau, \text { fin }}$.

\subsection{Proof of Theorem 5 (Pontrjagin-Thom construction)}

Start of the induction Suppose that $i=0$ ie $\tau=\left\{\eta_{0}\right\}$. Recall that $\left[\eta_{0}\right]$ is the class of germs of maximal rank. In this case a $\tau$-embedding is a usual embedding $e: M^{n} \hookrightarrow Q^{n+k+N}$ with normal vector fields $v_{1}, \ldots, v_{N}$ (defined on a neighbourhood of $e\left(M^{n}\right)$ ). Choose $V_{\tau}$ to be the Thom space $M S O(k)=T \gamma_{k}^{S O}$. (In this case $V_{\tau}$ exists as a space, not just as a virtual complex). The $N$-th suspension $S^{N} M S O(k)$ is the Thom space of the bundle $\gamma_{k}^{S O} \oplus \varepsilon^{N}$. Now the usual Pontrjagin-Thom construction provides all the statements of the Theorem. 
Induction step Let $\tau$ be equal to $\tau^{\prime} \cup\{\eta\}$, where $\eta$ is a top singularity type in $\tau$. Suppose that for $\tau^{\prime}$ we have constructed the virtual complex $V_{\tau^{\prime}}$ and all the statements of the Theorem including the Addenda are proved with $\tau^{\prime}$ substituted for $\tau$.

Let $e: M^{n} \hookrightarrow Q^{q}$ be a $(\tau, N)$-embedding $(q=n+k+N)$ and let $\eta(e)$ be the stratum of $\eta$-points of $e$. Then $\eta(e)$ is a proper submanifold in $M^{n}$ (this means that it is the image of a smooth embedding, it is a closed subset, and corners of codimension $i$ in $\eta(e)$ - and only they - lie in those of $M^{n}$ ). Let $\widetilde{T}$ be a small tubular neighbourhood of $e(\eta(e))$ in $Q^{q}$ and let $T$ be that of $\eta(e)$ in $M^{n}$. Since the $N$-dimensional foliation $\mathcal{F}$ is transverse to the stratum $e(\eta(e))$ the tubular neighbourhood $\widetilde{T}$ can be decomposed into the product $\widetilde{T}^{\perp} \times D^{N}$. The leaves of the foliation $\mathcal{F}$ are the sets $x \times D^{N}$, for $x \in \widetilde{T}^{\perp}$. Here $\widetilde{T}^{\perp}$ can be identified with a $D^{c+k}$-bundle over $e(\eta(e))$, its fibres are the images under the exponential map of the small $\varepsilon$-balls orthogonal to $V_{x} \oplus T_{x}(e(\eta(e))$ in $T_{x} Q^{q}$ for $x \in e(\eta(e))$. (Recall that $c+k$ is the dimension of the target of the root of $\eta$.) Let us denote by $\pi$ the projection $\widetilde{T} \rightarrow \widetilde{T}^{\perp}$ along the leaves of $\mathcal{F}$ (ie the projection of $\tilde{T}=\widetilde{T}^{\perp} \times D^{N}$ onto $\widetilde{T}^{\perp}$ ). Let us consider the map $e_{\eta}^{\perp}=\left.\pi \circ e\right|_{T}: T \rightarrow \widetilde{T}^{\perp}$. This is a proper $\tau$-map, having $\eta$ points at the zero-section of the bundle $T \rightarrow \eta(e)$. By Proposition 28 (about the normal form around a stratum) there is a commutative diagram

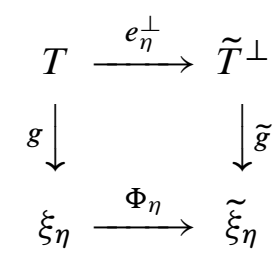

From now on we will write simply $\xi$ and $\tilde{\xi}$ omitting the subindex $\eta$. Using the fact that the bundles $T$ and $\widetilde{T}$ have finite-dimensional base spaces of dimension $\leq n$, we can replace the bundles $\xi$ and $\widetilde{\xi}$ by their finite-dimensional approximations $\xi_{\text {fin }}$ and $\widetilde{\xi}_{\text {fin }}$ over a finite-dimensional approximation of $B G_{\eta}$ denoted by $B G_{\eta \text {,fin }}$ so that (3) still holds. Let us denote its dimension by $\mu(n)$. We have seen that depending on the dimension $\mu(n)$ of this approximation there is a natural number $\widehat{N}=\hat{N}(\mu(n))$ such that there is an embedding (unique up to isotopy) $\xi_{\text {fin }} \rightarrow D^{\widehat{N}}$ of the total space of the bundle $\xi_{\text {fin }}$. (One can choose for $\hat{N}(\mu(n))$ the number $N(n)=2 \mu(n)+2 n+2$.) 
For $N \geq N(n)$, there is an embedding $j: \xi_{\text {fin }} \rightarrow D^{N}$ (unique up to isotopy). We denoted by $\Phi_{\eta \text {,fin }}^{\text {lift }}$ the embedding $\left(\Phi_{\eta, \text { fin }}, j\right): \xi_{\text {fin }} \rightarrow \widetilde{\xi}_{\text {fin }} \times D^{N}$.

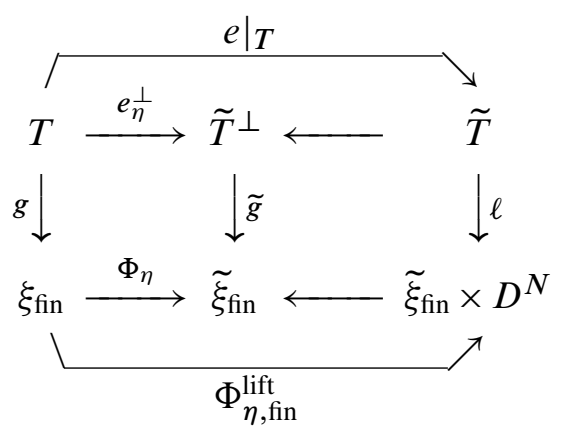

There is a map $\ell: \widetilde{T} \rightarrow \widetilde{\xi}_{\text {fin }} \times D^{N}$ that makes this diagram commutative.

The composition of the map $\ell: \widetilde{T} \rightarrow D(\tilde{\xi}) \times D^{N}$ with the inclusion $D(\tilde{\xi}) \times D^{N} \rightarrow$ $S^{N} V_{\tau, \text { fin }}$ together with the (stable) map $Q^{q} \backslash \widetilde{T} \rightarrow S^{N} V_{\tau^{\prime} \text {,fin }}$ corresponding to the $\tau^{\prime}$-embedding $\left.e\right|_{M^{n} \backslash T}: M^{n} \backslash T \rightarrow Q^{q} \backslash \widetilde{T}$ (where $T=e^{-1}(\widetilde{T})$ ) gives a stable map $\varphi: Q^{q} \rightarrow S^{N} V_{\tau}$. (The two maps obtained on the common boundary $\partial \widetilde{T}=\partial\left(Q^{q} \backslash \operatorname{int} \tilde{T}\right)$ are homotopic, because these are maps corresponding to the same $\left(\tau^{\prime}, N\right)$-embedding $\partial T \subset \partial \tilde{T}$. Then it can be achieved by a deformation that these maps coincide.)

The proof of the statement that stably cobordant $\tau$-embeddings give stably homotopic maps is quite similar. Thus the virtual complex $V_{\tau}$ and the map $\alpha$ have been constructed.

Next we construct the map

$$
\beta:\left\{\dot{Q}^{q}, S^{N} V_{\tau}\right\} \rightarrow \operatorname{Emb}_{(\tau, N)}^{\text {stable }}\left(Q^{q}\right) .
$$

According to Remark 66 given a map $\dot{Q}^{q} \rightarrow S^{N} V_{\tau \text {,fin }}$ we can pull back the $(\tau, N)$ embedding $\mathcal{K}_{\tau \text {,fin }} \subset S^{N} V_{\tau \text {,fin }}$ and then we obtain a $(\tau, N)$-embedding unique up to cobordism and this cobordism class depends only on the homotopy class of the given map.

In general (when the map $\dot{Q}^{q} \rightarrow S^{N} V_{\tau \text {,fin }}$ is given only stably, then) we have to replace $Q^{q}$ and $N$ by $Q^{q} \times R^{L}$ and $N+L$ respectively for a big enough $L$.

So we obtain a map

$$
\beta_{\text {fin }}:\left\{\dot{Q}^{q}, S^{N} V_{\tau, \text { fin }}\right\} \rightarrow \operatorname{Emb}_{(\tau, N)}^{\text {stable }}\left(Q^{q}\right) .
$$

It remained to note that the natural map

$$
\left\{\dot{Q}^{q}, S^{N} V_{\tau, \mathrm{fin}}\right\} \rightarrow\left\{\dot{Q}^{q}, S^{N} V_{\tau}\right\}
$$


is an isomorphism and then $\beta$ is the composition of $\beta_{\text {fin }}$ with this isomorphism. It is an isomorphism because the pair $\left(B G_{i}, B G_{i, \text { fin }}\right)$ is $(n+1)$-connected, hence the pair $\left(S^{N} V_{\tau}, S^{N} V_{\tau, \text { fin }}\right)$ is $(N+n+1)$-connected, so we can apply the generalised Freudenthal theorem.

Clearly the maps $\alpha$ and $\beta$ are each others inverses by construction.

The naturality properties also follow clearly from the constructions of the maps.

\subsection{Summary}

We have shown that given manifolds $P^{n+k}$ and $Q^{q}$, we have:

a) $\operatorname{Cob}_{\tau}\left(P^{n+k}\right)=\operatorname{Emb}_{(\tau, N)}\left(P^{n+k} \times \mathbb{R}^{N}\right)$ for any sufficiently large $N$.

b) For any sufficiently large $N, \operatorname{Emb}_{(\tau, N)}^{\text {stable }}\left(P^{n+k}\right) \approx \operatorname{Emb}_{(\tau, N)}\left(P^{n+k} \times \mathbb{R}^{N}\right)$.

c) $\operatorname{Emb}_{(\tau, N)}^{\text {stable }}\left(Q^{q}\right)=\left\{\dot{Q}^{q}, S^{N} V_{\tau}\right\}$, for any natural number $N$, where $V_{\tau}=\dot{\bigcup}\left\{D \widetilde{\xi}_{\eta} \mid\right.$ $[\eta] \in \tau\}$ is the virtual complex described above.

Corollary $67 \operatorname{Cob}_{\tau}\left(P^{n+k}\right) \approx\left\{P^{\dot{n}+k}, V_{\tau}\right\}$

\section{Remark 68}

1) Recall that a space $X_{\tau}$ was constructed satisfying the equality $\operatorname{Cob}_{\tau}\left(P^{n+k}\right)=$ $\left[P^{\dot{n}+k}, X_{\tau}\right]$ in [29].

2) We have seen that the functors $\operatorname{Cob}_{\tau}(-)$ and $\operatorname{Emb}_{\tau}\left(-\times \mathbb{R}^{N}\right)$ can be extended from manifolds to simplicial complexes. (See Definition 7 and also the author's paper [36]). The Corollary above will hold for any simplicial complex $P$.

We obtain the following:

Proposition $69 X_{\tau} \cong \Gamma V_{\tau}$, where $\Gamma()=\Omega^{\infty} S^{\infty}()$.

Proof We have

and

$$
\begin{aligned}
& \operatorname{Cob}_{\tau}(P) \approx\left\{\dot{P}, V_{\tau}\right\}=\left[\dot{P}, \Gamma V_{\tau}\right] \\
& \operatorname{Cob}_{\tau}(P)=\left[\dot{P}, X_{\tau}\right] .
\end{aligned}
$$

Hence both the space $X_{\tau}$ and the space $\Gamma V_{\tau}$ are classifying spaces for the functor $\mathrm{Cob}_{\tau}(-)$. But by Brown's theorem the classifying space is homotopically unique. 


\section{Part IV The Kazarian conjecture}

\section{Formulation of the conjecture}

Maxim Kazarian formulated a conjecture relating the space $\mathcal{K}_{\tau}$ (which we called Kazarian space) with the space $X_{\tau}$, classifying $\tau$-cobordisms.

Below $\cong \mathbb{Q}$ means "rationally homotopically equivalent" and $\mathcal{K}_{\tau}^{+}$denotes the disjoint union of $\mathcal{K}_{\tau}$ with a point.

Conjecture 70 (Kazarian [21])

$$
X_{\tau} \cong \mathbb{Q} \Gamma S^{k}\left(\mathcal{K}_{\tau}^{+}\right) .
$$

This means that $X_{\tau}$ gives the stable rational homotopy type of the $k$-th suspension of $\mathcal{K}_{\tau}^{+}$. Here we shall formulate and prove a statement giving a relationship between $X_{\tau}$ and $\mathcal{K}_{\tau}$ that gives a homotopy equivalence - not just a rational one - after modifying the right-hand side properly. Notice that $S^{k}\left(\mathcal{K}_{\tau}^{+}\right)$is the Thom space of the trivial $k-$ dimensional vector bundle over $\mathcal{K}_{\tau}$. Roughly speaking we replace the $k$-th suspension $S^{k}\left(\mathcal{K}_{\tau}^{+}\right)$by the Thom space of the $k$-dimensional vector bundle $\nu^{k}$ over $\mathcal{K}_{\tau}$; see Definition 25. But actually $v^{k}$ is only a virtual vector bundle.

Remark 71 Recall that a virtual vector bundle $(\alpha, \beta)$ over a space $A$ is an equivalence class of formal differences of vector bundles. By dimension of such a virtual vector bundle $(\alpha, \beta)$ one means the difference $\operatorname{dim}(\alpha, \beta)=\operatorname{dim}$ fibre of $\alpha-\operatorname{dim}$ fibre of $\beta$. If $A$ is compact, then any virtual vector bundle admits a representation of the form $\left(\alpha, \varepsilon^{N}\right)$, where $\varepsilon^{N}$ is the trivial $N$-bundle. If $A$ is a union of compact subspaces $A_{1} \subset A_{2} \subset \ldots, A=\bigcup A_{i}$, then a virtual bundle of dimension $k$ over $A$ is represented by a sequence $\left(\alpha_{i}, \varepsilon^{N_{i}}\right)$ of pairs, where $\alpha_{i}$ is a vector bundle over $A_{i}$ of dimension $N_{i}+k, \varepsilon^{N_{i}}$ is the trivial $N_{i}$-dimensional bundle and $\left.\alpha_{i}\right|_{A_{i-1}}=\alpha_{i-1} \oplus \varepsilon^{N_{i}-N_{i-1}}$.

Definition 72 By Thom space of a virtual bundle $\left(\alpha, \varepsilon^{N}\right)$ we mean the virtual complex $S^{(-N)} T \alpha$. Here $T \alpha$ is the Thom space of the bundle $\alpha$, and $S^{(-N)}$ is the inverse of the $N$-th suspension. Note that the $(-N)$-th suspension is a meaningful functor from virtual complexes into virtual complexes.

Proposition 73 There exists a virtual $k$-dimensional vector bundle $v^{k}$ over $\mathcal{K}_{\tau}$ such that $\Gamma V_{\tau} \cong \Gamma T \nu^{k} .^{8}$

${ }^{8} \cong$ stands for "homotopy equivalent". 
The next corollary is a refinement of Kazarian's conjecture.

Corollary $74 X_{\tau} \cong \Gamma T v^{k}$.

Proof It follows immediately from Propositions 73 and 69.

Proof of Proposition 73 We have seen that the bundles $D \widetilde{\xi}_{\eta \text {, fin }} \times D^{N}$ attached properly

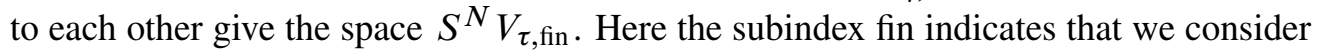
certain finite-dimensional approximations. From now on we will omit this subindex. The attaching maps are defined by the embeddings $D \xi_{\eta} \subset D \widetilde{\xi}_{\eta} \times D^{N}$. When we attach the bundle $D \widetilde{\xi}_{\eta} \times D^{N}$ to $S^{N} V_{\tau^{\prime}}$, then the bundle $D \xi_{\eta}$ is attached to $\mathcal{K}_{\tau^{\prime}} \subset S^{N} V_{\tau^{\prime}}$. So we obtain that $\mathcal{K}_{\tau} \subset S^{N} V_{\tau}$. Recall that $\nu^{k}$ is the virtual vector bundle over $\mathcal{K}_{\tau}$ which is defined by the following restrictions: $\left.v^{k}\right|_{D \xi_{\eta}}=\pi^{*} \widetilde{\xi}_{\eta}-\pi^{*} \xi_{\eta}$, where $\pi: D \xi_{\eta} \rightarrow B G_{\eta}$ is the disc-bundle of the bundle $\xi_{\eta}$. Note that for those finite-dimensional approximations where the embedding $D \xi_{\eta \text {,fin }} \hookrightarrow D \widetilde{\xi}_{\eta \text {,fin }} \times D^{N}$ exists the virtual bundle $v^{k} \oplus \varepsilon^{N}$ can be realized as a (nonvirtual) vector bundle (it can be regarded as the "normal bundle" of the embedding $\mathcal{K}_{\tau \text {,fin }} \hookrightarrow S^{N} V_{\tau \text {,fin }}$ ). Both the space $\mathcal{K}_{\tau}$ and the virtual complex $V_{\tau}$ are stratified by their constructions:

$$
\mathcal{K}_{\tau}=\bigcup\left\{D \xi_{\eta_{i}} \mid\left[\eta_{i}\right] \in \tau\right\} ; \quad V_{\tau}=\bigcup^{\bullet}\left\{D \tilde{\xi}_{\eta_{i}} \mid\left[\eta_{i}\right] \in \tau\right\}
$$

The inclusion $\mathcal{K}_{\tau} \subset S^{N} V_{\tau}$ respects this stratification:

$$
\left(\mathcal{K}_{\tau}, \mathcal{K}_{\tau^{\prime}}\right) \subset\left(S^{N} V_{\tau}, S^{N} V_{\tau^{\prime}}\right)
$$

Hence it induces map of the quotient spaces

$$
i_{\eta}: T \xi_{\eta} \hookrightarrow S^{N} T \widetilde{\xi}_{\eta} .
$$

(Here $\eta$ is a highest singularity in $\tau$ and $\tau^{\prime}=\tau \backslash\{\eta\}$.) The base space of both bundles $\xi_{\eta}$ and $\widetilde{\xi}_{\eta}$ is the space $B G \eta$ and $i_{\eta}$ is the identity on it.

Let $U$ be a tubular neighbourhood of $\mathcal{K}_{\tau}$ in $S^{N} V_{\tau}$. Then the map

$$
S^{N} V_{\tau} \rightarrow S^{N} V_{\tau} /\left(S^{N} V_{\tau} \backslash U\right)=S^{N} T \nu^{k}
$$

induces maps of the quotients

$$
\begin{aligned}
& S^{N} V_{\tau} / S^{N} V_{\tau^{\prime}}=S^{N} T \tilde{\xi}_{\eta}=T\left(\tilde{\xi}_{\eta} \oplus \varepsilon^{N}\right) \\
& \quad \rightarrow S^{N} T \widetilde{\xi}_{\eta} /\left(S^{N} T \widetilde{\xi} \backslash U\right)=S^{N} T\left(\left.v^{k}\right|_{D \xi_{\eta}}\right) / S^{N} T\left(\left.v^{k}\right|_{\partial D \xi_{\eta}}\right)=T\left(v^{k} \oplus \xi_{\eta} \oplus \varepsilon^{N}\right) .
\end{aligned}
$$

These maps map the zero section of $\widetilde{\xi}_{\eta} \oplus \varepsilon^{N}$ into that of $v \oplus \xi_{\eta} \oplus \varepsilon^{N}$ by the identity homeomorphism. Therefore they induce isomorphisms in the cohomologies. Hence the map (4) also induces isomorphism on cohomologies. 
Remark 75 Note that in (4) we actually have some finite dimensional approximation $V_{\tau \text {,fin }}$ of the virtual complex $V_{\tau}$ and the natural number $N$ is chosen so that:

a) $S^{N} V_{\tau, \text { fin }}$ exists as a (nonvirtual) space.

b) Over the corresponding finite dimensional approximation of $\mathcal{K}_{\tau}$ the virtual bundle $v^{k}$ has the form $\alpha^{N+k}-\varepsilon^{N}$.

So the Thom space $S^{N} T v^{k}$ (as a virtual complex) has the corresponding finite dimensional approximation $T \alpha^{N+k}$. We have shown that $S^{N} V_{\tau, \text { fin }}=T \alpha^{N+k}$. Therefore the virtual complexes $V_{\tau}$ and $T v^{k}$ have sequences of finite dimensional approximations and there exist natural numbers $N_{j}$ such that the $N_{j}$-th suspensions of the $j$-th approximations exist and are homotopically equivalent. We express this by saying that the virtual complexes $V_{\tau}$ and $T \nu^{k}$ are homotopically equivalent and write: $V_{\tau} \dot{\cong} T \nu^{k}$. In particular $\Gamma V_{\tau} \cong \Gamma T \nu^{k}$.

Notation 76 For any space $A$ let us denote by $S P A$ the infinite symmetric product of $A$.

Remark 77 It is known (see eg Hatcher [15, page 472]) that for any base pointed, connected $C W$-complex $A$ the space $S P A$ is (homotopy equivalent to) a product of Eilenberg-MacLane spaces. Since by the Dold-Thom theorem $\pi_{i}(S P A)=H_{i}(A)$ (see again Hatcher [15]), we obtain

$$
S P A=\prod_{i} K\left(H_{i}(A), i\right) .
$$

Lemma 78 Let $A$ and $B$ be arbitrary spaces such that $H_{*}(A) \approx H_{*}(B)$. Then $S P A$ is weakly homotopy equivalent to $S P B$.

Proof Obvious from (5).

Remark 79 We do not require that $A$ and $B$ were simply connected, neither that the isomorphism $H_{*}(A) \approx H_{*}(B)$ were induced by a map of the spaces.

\section{Remark 80}

1) In particular $\Omega(S P(S A))=S P A$.

2) A cofibration $B \subset A \rightarrow A / B$ gives a quasi-fibration (see [15]) of the corresponding infinite symmetric products $S P B \rightarrow S P A \rightarrow S P(A / B)$ for which the homotopy exact sequence holds. Hence if the pair $(A, B)$ is $\ell$-connected, then the pair $(S P A, S P B)$ is also at least $\ell$-connected. 
3) Therefore the functor $S P($ ) can be defined for a virtual complex $V$ as well as follows: Let $\left(m_{j}, n_{j}\right)$ be a sequence of pairs of natural numbers from Definition 61 , ie $S^{n_{j}}$ sk $_{m_{j}} V$ exists, $n_{j} \rightarrow \infty, m_{j} \rightarrow \infty$. Now put

$$
S P(V)=\lim _{j \rightarrow \infty} \Omega^{n_{j}} S P S^{n_{j}} \mathrm{sk}_{m_{j}} V .
$$

\section{Proof of the Kazarian conjecture}

By the Thom isomorphism we have

$$
\bar{H}^{*+k}\left(T v^{k}\right) \approx H^{*}\left(\mathcal{K}_{\tau}\right) \approx \bar{H}^{*+k}\left(S^{k}\left(\mathcal{K}_{\tau}^{+}\right)\right) .
$$

We obtain the following equalities:

$$
X_{\tau}=\Gamma V_{\tau}=\Gamma T \nu^{k} \text { and } S P T \nu^{k}=S P\left(S^{k}\left(\mathcal{K}_{\tau}^{+}\right)\right) .
$$

Here equality means homotopy equivalence.

Lemma 81 For any (virtual) complex $A$ there is a natural map $h^{A}: \Gamma A \rightarrow S P A$ that induces a rational homotopy equivalence. Moreover the map $h_{\#}^{A}$ induced by $h^{A}$ in the homotopy groups is the stable Hurewicz map $h_{\#}^{A}: \pi_{i}(\Gamma A)=\pi_{i}^{s}(A) \rightarrow H_{i}(A)=$ $\pi_{i}(S P A)$.

Proof In [6] a model has been constructed for the space $\Gamma A$ for any connected complex $A$ :

$$
\Gamma A=\coprod_{i} W S(i) \underset{S(i)}{\times}(A \times \cdots \times A) / \sim
$$

where $W S(i)$ is a contractible space with a free $S(i)$-action on it, and $\sim$ denotes gluing by some natural equivalences. ( $S(i)$ is the symmetric group on $i$ elements.) The projections

$$
W S(i) \underset{S(i)}{\times}(A \times \cdots \times A) \rightarrow(A \times \cdots \times A) / S(i)
$$

are consistent for different $i$ 's with the gluing in $\Gamma(A)$ and $S P(A)$. So they define a map $h: \Gamma(A) \rightarrow S P(A)$ and the induced map $h_{\#}^{A}: \pi_{i}(\Gamma(A))=\pi_{i}^{S}(A) \rightarrow$ $\pi_{i}^{S}(S P(A))=H_{i}(A)$ is the stable Hurewicz map. By Serre's theorem this is a rational isomorphism (see Theorem 18.3 in [27]). This proves the lemma when $A$ is a nonvirtual space. If $A$ is a virtual complex, then we replace it by its appropriate suspension (over each finite skeleton) and repeat the previous proof using that $\Omega^{N} \Gamma S^{N} A=\Gamma A$ and $\Omega^{N} S P S^{N} A=S P A$. 
Now we finish the proof of the Kazarian conjecture:

$$
X_{\tau}(k) \stackrel{(1)}{\cong} \Gamma T \nu^{k} \stackrel{(2)}{\cong} \mathbb{Q} S P T \nu^{k} \stackrel{(3)}{=} \operatorname{SPT} \varepsilon^{k} \stackrel{(4)}{=} \operatorname{SP} S^{k}\left(\mathcal{K}_{\tau}^{+}\right) \stackrel{(5)}{\cong} \mathbb{Q} \Gamma S^{k}\left(\mathcal{K}_{\tau}^{+}\right)
$$

Here (1) is Corollary 74, (2) and (5) follow from Lemma 81, (3) comes from Lemma 78 and (4) is obvious.

Remark 82 It follows from the above proof that the following modification of the Kazarian conjecture also holds: ${ }^{9}$

$$
X_{\tau} \cong \mathbb{Q} S P\left(S^{k}\left(\mathcal{K}_{\tau}^{+}\right)\right) .
$$

\section{Corollaries of the Kazarian conjecture}

Here we compute the rational cobordism groups of $\tau$-maps. Further we define characteristic classes of $\tau$-maps, show analogues of the Pontrjagin-Thom theorem and those of the Conner-Floyd theorem claiming that the characteristic numbers completely determine the cobordism (respectively bordism) class.

Proposition $83 \operatorname{Cob}_{\tau}(n, k) \otimes \mathbb{Q} \approx H_{n}\left(\mathcal{K}_{\tau} ; \mathbb{Q}\right)$

Proof We have

$$
\begin{aligned}
\operatorname{Cob}_{\tau}(n, k) \otimes \mathbb{Q} & =\pi_{n+k}\left(X_{\tau}\right) \otimes \mathbb{Q}=\pi_{n+k}\left(\Gamma V_{\tau}\right) \otimes \mathbb{Q}=\pi_{n+k}^{s}\left(V_{\tau}\right) \otimes \mathbb{Q} \\
& =\pi_{n+k}^{s}\left(T v^{k}\right) \otimes \mathbb{Q} \stackrel{h_{\mathbb{Q}}}{\longrightarrow} H_{n+k}\left(T v^{k} ; \mathbb{Q}\right)=H_{n}\left(\mathcal{K}_{\tau} ; \mathbb{Q}\right),
\end{aligned}
$$

where $h_{\mathbb{Q}}$ is the tensor product of the stable Hurewicz homomorphism with the identity $\operatorname{map} \operatorname{id}_{\mathbb{Q}}: \mathbb{Q} \rightarrow \mathbb{Q}$, hence it is an isomorphism.

So the stable homotopy groups of the virtual complex $V_{\tau}$ give the $\tau$-cobordism groups, while the homology groups of $V_{\tau}$ are isomorphic to those of $\mathcal{K}_{\tau}$ after a shift of the dimensions by $k$. Now we are going to give a more explicit description of the arising map $\operatorname{Cob}_{\tau}(n, k) \rightarrow H_{n}\left(\mathcal{K}_{\tau}\right)$, denote this map by $\varphi$.

Proposition 84 Let $f: M^{n} \rightarrow \mathbb{R}^{n+k}$ be a $\tau$-map, and let $\kappa_{f}: M^{n} \rightarrow \mathcal{K}_{\tau}$ be the corresponding Kazarian map, lifting the stable normal map $v_{f}: M^{n} \rightarrow B S O$. Then $\varphi([f])=\left(\kappa_{f}\right)_{*}[M]$.

Proof This is standard; see the arXiv version 1 [41, page 34] of this paper.

\footnotetext{
${ }^{9}$ Kazarian recently has told me that this was the first version of his conjecture.
} 
Remark 85 This Proposition can be considered as a (rational) analogue for $\tau$-maps of the Pontrjagin-Thom theorem saying that two manifolds are cobordant if and only if their characteristic numbers coincide. To make the analogy more transparent we give the definition of characteristic numbers of a $\tau$-map and reformulate the Proposition.

Definition 86 If $f: M^{n} \rightarrow \mathbb{R}^{n+k}$ is a $\tau$-map, and $x \in H^{n}\left(\mathcal{K}_{\tau}\right)$ is any $n$-dimensional cohomology class of $\mathcal{K}_{\tau}$, then the number $\left\langle\kappa_{f}^{*}(x),\left[M^{n}\right]\right\rangle$ will be called the $x$-characteristic number of $f$.

Proposition 87 There is an isomorphism

$$
\begin{aligned}
\operatorname{Cob}_{\tau}(n, k) \otimes \mathbb{Q} & \stackrel{\approx}{\longrightarrow} \operatorname{Hom}\left(H^{n}\left(\mathcal{K}_{\tau}\right) ; \mathbb{Q}\right) \\
\psi:[f] & \longmapsto\left(x \longmapsto\left\langle\kappa_{f}^{*}(x),\left[M^{n}\right]\right\rangle\right) .
\end{aligned}
$$

Proof $\left\langle\kappa_{f}^{*}(x),\left[M^{n}\right]\right\rangle=\left\langle x, \kappa_{f *}\left[M^{n}\right]\right\rangle$. The set of these numbers for $\forall x \in H^{n}\left(\mathcal{K}_{\tau}\right)$ define the class $\kappa_{f *}\left[M^{n}\right]$. Hence the map $\psi$ is injective. Its source and target have equal dimensions over $Q$, hence $\psi$ is an isomorphism.

Example 88 Let $\tau$ be the set of all possible singularities of codimension $k$ germs. Then $\operatorname{Cob}_{\tau}(n, k)=\Omega_{n}\left(\mathbb{R}^{n+k}\right)=\Omega_{n}$, where $\Omega_{n}(X)$ denotes the $n$-th bordism group of a space $X$ and $\Omega_{n}$ is the cobordism group of oriented $n$-dimensional manifolds. In this case $\mathcal{K}_{\tau} \cong B S O=\lim _{N \rightarrow \infty} B S O(N)$. (The fibre of the map $\mathcal{K}_{\tau} \rightarrow B S O$ is the space of all polynomial maps and that is contractible.) So in this case our proposition says:

$$
\Omega_{n} \otimes \mathbb{Q} \approx \operatorname{Hom}\left(H^{n}(B S O) ; \mathbb{Q}\right) \approx H_{n}(B S O ; \mathbb{Q}),
$$

and that is a well-known theorem of Thom. ${ }^{10}$

Example 89 Let $\tau$ be equal to $\left\{\Sigma^{0}\right\}$ ie in this case a $\tau$-map is the same as an immersion. Then $\mathcal{K}_{\tau}$ is the $V_{N}\left(\mathbb{R}^{N+k}\right)$ bundle associated to the bundle $\gamma_{N+k}^{S O} \rightarrow$ $B S O(N+k)$, for $N \rightarrow \infty$.

But this is just $B S O(k)$. Hence the Proposition says that the rational cobordism group of oriented immersions $\operatorname{Cob}_{\tau}(n, k) \otimes \mathbb{Q}=\operatorname{Imm}^{S O}(n, k) \otimes \mathbb{Q}$ is isomorphic to $H_{n}(B S O(k) ; \mathbb{Q})$. (Compare with Burlet [7].)

Next we study the case when the target is an arbitrary oriented $(n+k)-$ manifold $P^{n+k}$.

${ }^{10}$ This case is not quite covered by our consideration, since we allowed in $\tau$ only stable singularities. 
Proposition $90 \operatorname{Cob}_{\tau}\left(P^{n+k}\right) \otimes \mathbb{Q} \approx \bigoplus_{j} H^{j}\left(P^{n+k} ; H_{j-k}\left(\mathcal{K}_{\tau} ; \mathbb{Q}\right)\right)$.

Proof We will use the sign " $A \approx \mathbb{Q}_{\mathbb{Q}} B$ " meaning that $A \otimes \mathbb{Q} \approx B \otimes \mathbb{Q}$, where $A$ and $B$ are Abelian groups. (Further we recall that for any space $X$ we have $S P X=$ $\prod_{j} K\left(H_{j}(X), j\right)$ and there is a weak rational homotopy equivalence $h^{X}: \Gamma X \rightarrow$ $S P X$, where $\Gamma X=\Omega^{\infty} S^{\infty} X$.)

$$
\begin{aligned}
\operatorname{Cob}_{\tau}\left(P^{n+k}\right) & =\left[P^{n+k}, X_{\tau}\right]=\left[P^{n+k}, \Gamma V_{\tau}\right]=\left[P^{n+k}, \Gamma T v^{k}\right] \underset{\approx_{\mathbb{Q}}}{\stackrel{h^{T v^{k}}}{\longrightarrow}}\left[P^{n+k}, S P T v^{k}\right] \\
& =\left[P^{n+k}, \prod_{j} K\left(H_{j}\left(T v^{k}\right), j\right)\right]=\left[P^{n+k}, \prod_{j} K\left(H_{j-k}\left(\mathcal{K}_{\tau}\right), j\right)\right] \\
& =\bigoplus_{j} H^{j}\left(P^{n+k} ; H_{j-k}\left(\mathcal{K}_{\tau}\right)\right) .
\end{aligned}
$$

Definition 91 We will call the cohomology classes in $H^{*}\left(\mathcal{K}_{\tau}\right)$ characteristic classes of $\tau$-maps. If $f$ is a $\tau$-map, $\kappa_{f}: M^{n} \rightarrow \mathcal{K}_{\tau}$ is the corresponding Kazarian map and $x \in H^{*}\left(\mathcal{K}_{\tau}\right)$, then $\kappa_{f}^{*}(x)$ will be called the $x$-characteristic class of the $\tau$-map $f$.

Definition 92 Let $f: M^{n} \rightarrow P^{n+k}$ be a $\tau$-map, representing a cobordism class $[f] \in \operatorname{Cob}_{\tau}\left(P^{n+k}\right)$. Let $x$ be a cohomology class in $H^{*}\left(\mathcal{K}_{\tau}\right)$ and $z \in H^{*}\left(P^{n+k}\right)$. Then the number $\left\langle\kappa_{f}^{*}(x) \cup f^{*}(z),\left[M^{n}\right]\right\rangle$ will be called the $(x, z)$-characteristic number of the $\tau$-map $f$.

Proposition 93 Let $f: M^{n} \rightarrow P^{n+k}$ be a $\tau$-map. Then the $\tau$-characteristic numbers $\left\langle\kappa_{f}^{*}(x) \cup f^{*}(z),\left[M^{n}\right]\right\rangle$ determine the rational $\tau$-cobordism class of $f$, ie $[f] \otimes 1_{\mathbb{Q}} \in$ $\operatorname{Cob}_{\tau}\left(P^{n+k}\right) \otimes \mathbb{Q}$, and they are well-defined invariants of this class. Moreover, if for any $x \in H^{*}\left(\mathcal{K}_{\tau}\right)$ and $z \in H^{*}\left(P^{n+k}\right)$ a rational number $r(x, z)$ is given, then there is a unique element in $\operatorname{Cob}_{\tau}\left(P^{n+k}\right) \otimes \mathbb{Q}$ such that the numbers $r(x, z)$ give the $(x, z)$-characteristic numbers of this element.

Remark 94 Following the suggestion of the referee I omit the proof here (but refer to [41, page 37], where one can find also an application of these characteristic numbers, namely the ring structure on the direct sum of all the cobordism groups of fold maps, sum for all dimensions and all codimensions, has been computed [41, Part IX].). This will be published elsewhere; see the preprint of Lippner and the author [25]. 


\section{Part V Concrete computations}

Here we compute explicitly the rational cobordism groups of

a) Morin maps with trivial (and trivialised) kernel bundle (these are the so called prim maps),

b) arbitrary Morin maps,

c) Morin maps with at most $\Sigma^{1_{r}}$ singularities.

\section{Morin maps}

Let $\tau$ be the set of all stable singularity classes of corank at most 1 , that is $\tau=$ $\left\{\Sigma^{0}, \Sigma^{1,0}, \ldots, \Sigma^{1_{i}, 0}, \ldots\right\}$.

Here $1_{i}$ stands for $i$ digits 1 , and any Thom-Boardman symbol stands for the corresponding stable singularity class. Such a $\tau$-map is called a Morin map. For this $\tau$ the group $\operatorname{Cob}_{\tau}(n, k)$ will be denoted by $\operatorname{Cob}_{\text {Morin }}(n, k)$. In this section we prove the following:

\section{Theorem 6}

$$
\operatorname{Cob}_{\text {Morin }}(n, k) \otimes \mathbb{Q}= \begin{cases}H_{n}(B O(k) ; \mathbb{Q}) & \text { if } k \text { is even }, \\ H_{n}(B O(k+1) ; \mathbb{Q}) & \text { if } k \text { is odd }\end{cases}
$$

Remark 95 Recall that we always consider oriented cobordism group of singular maps. Surprisingly these are related to the unoriented Grassmann manifolds.

\section{Lemma 96}

$$
\mathcal{K}_{\text {Morin }}(k) \cong \mathbb{Q} \begin{cases}B O(k) & \text { if } k \text { is even }, \\ B O(k+1) & \text { if } k \text { is odd }\end{cases}
$$

Since $\operatorname{Cob}_{\tau}(n, k) \otimes \mathbb{Q} \approx H_{n}\left(\mathcal{K}_{\tau} ; \mathbb{Q}\right)$ Lemma 96 implies Theorem 6. The proof of the Lemma consists of computing Kazarian's spectral sequence for Morin maps. The main tool will be the notion of "prim maps".

Definition 97 We say that a Morin map $f: M^{n} \rightarrow P^{n+k}$ is a prim map (the word prim is an abbreviation for projected immersion) if the line bundle over the singularity set formed by the kernels of the differential is trivial and trivialised. Such a map always can be decomposed as $\pi \circ g$, where $g: M^{n} \rightarrow P^{n+k} \times \mathbb{R}^{1}$ is an immersion, and $\pi: P^{n+k} \times \mathbb{R}^{1} \rightarrow \mathbb{R}^{1}$ is the projection. Here the immersion $g$ is unique up to regular homotopy (if we require that $\partial(\pi \circ g) / \partial \underline{v}>0$, where $\underline{v}$ is the positive direction of $\operatorname{ker} d f)$. 
Remark 98 Let us denote by $\mathcal{K}_{\text {prim }}(k)$ the Kazarian space for all prim maps of codimension $k$. Similar to the decomposition $\mathcal{K}_{\text {Morin }}(k)=\bigcup_{i} D \xi_{i}$, where $\xi_{i}$ is the universal normal bundle in the source for the singularity stratum $\Sigma^{1_{i}}$, there is a decomposition $\mathcal{K}_{\text {prim }}(k)=\bigcup_{i} D \bar{\xi}_{i}$, where $D \bar{\xi}_{i} \rightarrow D \xi_{i}$ is a double covering for each $i>0,{ }^{11}$ and it is the identity of $B S O(k)=D \bar{\xi}_{0}=D \xi_{0}$ for $i=0$. Hence there is a $\mathbb{Z}_{2}$ action on $\mathcal{K}_{\text {prim }}(k)$ such that the quotient is $\mathcal{K}_{\text {Morin }}(k)$.

Prim maps are easy to handle because the automorphism groups (in the case of oriented source and target manifolds) are all isomorphic to $S O(k)$, and the corresponding bundles $\bar{\xi}_{i}$ (the universal normal bundle of the $\Sigma^{1_{i}}$-stratum in the source) are the bundles $i \cdot \gamma_{k} \oplus \varepsilon^{i}$, where $\varepsilon^{i}$ is the $i$-dimensional trivial bundle.

Lemma 99 Let $\bar{K}$ be a $C W$ complex with a cellular $\mathbb{Z}_{2}$-action and let $K$ be the quotient $\bar{K} / \mathbb{Z}_{2}$, and let $p: \bar{K} \rightarrow K$ be the quotient map. Suppose that $K$ is simply connected. Then $H^{*}(K ; \mathbb{Q})$ is equal to $H^{*}(\bar{K} ; \mathbb{Q})^{\mathbb{Z}_{2}}$ the $\mathbb{Z}_{2}$-invariant part of $H^{*}(\bar{K} ; \mathbb{Q})$, and the homomorphism $p^{*}: H^{*}(K ; \mathbb{Q}) \rightarrow H^{*}(\bar{K} ; \mathbb{Q})$ is injective and its image is $H^{*}(\bar{K} ; \mathbb{Q})^{\mathbb{Z}_{2}}$.

Proof Let us consider the map $q: \bar{K} \times_{\mathbb{Z}_{2}} S^{\infty} \rightarrow K=\bar{K} / \mathbb{Z}_{2}$. Note that $q^{-1}(x)=S^{\infty}$ if $x$ is not a fix point of the $\mathbb{Z}_{2}$-action, and $q^{-1}(x)=R P^{\infty}$ otherwise. Recall that there exists a Leray spectral sequence (see Godement [12]):

$$
E_{2}^{i, j}=H^{i}\left(K ; \mathcal{H}^{j}\left(q^{-1}(x) ; \mathbb{Q}\right)\right) \Longrightarrow H^{*}\left(\bar{K} \times_{\mathbb{Z}_{2}} S^{\infty} ; \mathbb{Q}\right) .
$$

Here $\mathcal{H}^{j}\left(q^{-1}(x) ; \mathbb{Q}\right)$ is the group of local coefficients at the point $x \in K$. Since $H^{j}\left(q^{-1}(x) ; \mathbb{Q}\right)=0$ for $j>0$ and $H^{0}\left(q^{-1}(x) ; \mathbb{Q}\right)=\mathbb{Q}$ we have that

$$
E_{2}^{i, j}=0 \text { for } j>0, \text { and } E_{2}^{i, 0}=H^{i}(K ; \mathbb{Q}) .
$$

So the Leray spectral sequence degenerates and gives that

$$
H^{i}\left(\bar{K} \times_{\mathbb{Z}_{2}} S^{\infty} ; \mathbb{Q}\right)=H^{i}(K ; \mathbb{Q}) .
$$

On the other hand, there is a fibration $\bar{K} \times_{\mathbb{Z}_{2}} S^{\infty} \stackrel{\bar{K}}{\longrightarrow} R P^{\infty}$, and now we consider the spectral sequence of this fibration: $E_{2}^{i, j}=H^{i}\left(R P^{\infty} ; \mathcal{H}^{j}(\bar{K} ; \mathbb{Q})\right)$ with twisted coefficients. The fundamental group $\pi_{1}\left(R P^{\infty}\right)=\mathbb{Z}_{2}$ acts on $\mathcal{H}^{*}(\bar{K} ; \mathbb{Q})$. Let us denote by $\mathcal{H}_{+}$and $\mathcal{H}_{-}$, respectively the eigenspaces for the eigenvalues +1 and -1 ,

${ }^{11}$ This double cover is associated to the kernel line bundle of a Morin map. Note that for a prim map the kernel line bundle is always trivial. 
respectively of the $\mathbb{Z}_{2}$-action. It is well known that

$$
\begin{aligned}
& H^{*}\left(R P^{\infty} ; \mathcal{H}_{-}^{*}\right)=0, \\
& H^{i}\left(R P^{\infty} ; \mathcal{H}_{+}^{j}\right)= \begin{cases}\mathcal{H}_{+}^{j} & \text { if } i=0 \\
0 & \text { if } i \neq 0 .\end{cases} \\
& E_{2}^{i, j}= \begin{cases}0 & \text { if } i \neq 0 \\
H^{j}(\bar{K} ; \mathbb{Q})^{\mathbb{Z}_{2}} & \text { if } i=0 .\end{cases} \\
& H^{j}\left(\bar{K} \times_{\mathbb{Z}_{2}} S^{\infty} ; \mathbb{Q}\right)=H^{j}(\bar{K} ; \mathbb{Q})^{\mathbb{Z}_{2}} .
\end{aligned}
$$

Hence

Hence

Now this last equality combined with (6) implies the Lemma.

Remark 100 If $K^{\prime}$ is another space with a $\mathbb{Z}_{2}$-action and $f: K^{\prime} \rightarrow \bar{K}$ is an equivariant map, then the induced map $f_{*}$ in the homologies respects the decomposition according to the eigenvalues +1 and -1 of the $\mathbb{Z}_{2}$-action. This implies that if

$$
\bar{K}_{0} \subset \bar{K}_{1} \subset \cdots \subset \bar{K}_{i} \subset \cdots \subset \bar{K}
$$

is a filtration by $\mathbb{Z}_{2}$-invariant subspaces, then the whole spectral sequence decomposes according to the eigenvalues +1 and -1 . (Indeed, apply the present Remark to $K^{\prime}=\bar{K}_{i}$.)

In particular, if $K_{i}=\bar{K}_{i} / \mathbb{Z}_{2}$, then the cohomological spectral sequence of the filtration

$$
K_{0} \subset K_{1} \subset \cdots \subset K_{i} \subset \cdots \subset K
$$

can be identified with the $\mathbb{Z}_{2}$-invariant part of the cohomological spectral sequence of (7). In particular if the spectral sequence of (7) degenerates, then so does that of (8), too.

In the next two subsections we shall apply these observations to $\bar{K}=\mathcal{K}_{\text {prim }}(k)$ and $K=\mathcal{K}_{\text {Morin }}(k)$.

\subsection{The spectral sequence for the Kazarian space $\mathcal{K}_{\text {prim }}(k)$ of prim maps}

\section{Lemma 101}

a) If the codimension $k$ of the maps is even, $k=2 \ell$, and $A=\mathbb{Q}\left[p_{1}, \ldots, p_{\ell}\right]$, then the spectral sequence for prim maps is the following:

\begin{tabular}{c|c|c|c|c}
$i=$ & 0 & 1 & 2 & 3 \\
\hline $\bar{E}_{1}^{i, *}$ & $A \oplus \chi A$ & $\bar{U}_{1} \cup(A \oplus \chi A)$ & $\bar{U}_{2} \cup(A \oplus \chi A)$ & $\bar{U}_{3} \cup(A \oplus \chi A)$ \\
\hline $\bar{E}_{2}^{i, *}=\bar{E}_{\infty}^{i, *}$ & $A$ & 0 & 0 & 0
\end{tabular}


where $\chi$ is the Euler class of the universal oriented $k$-bundle $\gamma_{k}^{S O}$, and $\bar{U}_{p}$ is the Thom class of the bundle $\bar{\xi}_{i}=i \cdot\left(\gamma_{k}^{S O} \oplus \varepsilon^{1}\right)$.

b) If $k$ is odd, $k=2 \ell+1$, then $\bar{E}_{1}^{i, *}=\bar{U}_{i} \cup H^{*}(B S O(k))$ and all the differentials vanish. Hence $\bar{E}_{\infty}^{i, *}=\bar{E}_{1}^{i, *}$.

Remark 102 The space $\mathcal{K}_{\text {prim }}(k)$, which is the Kazarian space for all oriented prim maps is equal to the space $B S O(k+1)$. (To a prim map in codimension $k$ it can be associated an immersion of codimension $(k+1)$, unique up to regular homotopy, its lift.) So we know that the spectral sequence converges to $H^{*}(B S O(k+1)$; $\mathbb{Q})$.

Proof for $\boldsymbol{k}=\mathbf{2} \boldsymbol{\ell}$ The inclusion $B S O(k) \subset T i \gamma_{k}$ induces an injection $H^{*}\left(T i \gamma_{k}\right) \rightarrow$ $H^{*}(B S O(k))$ with image equal to the ideal generated by $\chi^{i}$.

Hence we can rewrite the $\bar{E}_{1}$-term of the spectral sequence as follows:

\begin{tabular}{c|c|c|c|c|c}
$i=$ & 0 & 1 & 2 & 3 & \\
\hline$E_{1}^{i, *}$ & $A \oplus \chi A$ & $\chi A \oplus \chi^{2} A$ & $\chi^{2} A \oplus \chi^{3} A$ & $\chi^{3} A \oplus \chi^{4} A$ &
\end{tabular}

This spectral sequence converges to $H^{*}(B S O(k+1))$ which can be identified with the subring $A$ in $E_{1}^{0, *}=A \oplus \chi A$. By dimensional reason we have that the differential

$$
d_{1}: E_{1}^{0, k} \rightarrow E_{1}^{1, k} \text { maps the Euler class } \chi \text { to } c \chi \text { for } c \neq 0, c \in \mathbb{Q} .
$$

(Indeed, all the further differentials $d_{r}, r>1$ map the group $E_{r}^{0, k}$ into a trivial group. Hence the only chance for the Euler class to be killed is if its first differential is nontrivial.) Similarly, $d_{1}: E_{1}^{i, k} \rightarrow E_{1}^{i+1, k}$ maps $\chi^{i}$ to a nonzero multiple of $\chi^{i}$.

Lemma $103 d_{1}$ maps $\chi^{i+1} \cdot A \subset E_{1}^{i, *}$ onto $\chi^{i+1} \cdot A \subset E_{1}^{i+1, *}$ isomorphically.

Proof It is enough to show that $d_{1}\left(\chi \cdot p_{I}\right)=d_{1}(\chi) \cup p_{I}$ for any monomial $p_{I}$ of the Pontrjagin classes. Let us denote by $\overline{\mathcal{K}}_{r}$ the Kazarian space of the prim $\Sigma^{1_{r}}$ maps (ie prim maps having only $\Sigma^{0}, \Sigma^{1,0}, \ldots, \Sigma^{1_{r}}$ type singularities), then

$$
\overline{\mathcal{K}}_{r}=\bigcup_{i \leq r} D\left(i\left(\gamma_{k} \oplus \varepsilon^{1}\right)\right) .
$$

(For $i=0$ we put $D\left(i\left(\gamma_{k} \oplus \mathcal{E}^{1}\right)\right)=B S O(k)$.) The differential $d_{1}: E_{1}^{i, *} \rightarrow E_{1}^{i+1, *}$ is the boundary operator $\delta$ in the exact sequence of the triple $\left(\overline{\mathcal{K}}_{i+1}, \overline{\mathcal{K}}_{i}, \overline{\mathcal{K}}_{i-1}\right)$, by definition. The Pontrjagin classes $p_{1}, \ldots, p_{\ell}$ in $E_{1}^{p, *}$ can be identified with the Pontrjagin classes of the virtual normal bundle $v^{k}$ over $\overline{\mathcal{K}}_{\infty}$ restricted to the base space 
of $D\left(i\left(\gamma_{k} \oplus \varepsilon^{1}\right)\right) \subset \overline{\mathcal{K}}_{\infty}$. Hence the inclusion $j: \overline{\mathcal{K}}_{i} \hookrightarrow \overline{\mathcal{K}}_{i+1}$ maps them into each other: $j^{*}\left(p_{\alpha}\right)=p_{\alpha}$ for $\alpha=1, \ldots, \ell$. Now

$$
d_{1}\left(\chi \cup p_{I}\right)=\delta\left(\chi \cup p_{I}\right)=\delta\left(\chi \cup j^{*}\left(p_{I}\right)\right)=\delta(\chi) \cup p_{I}+\chi \cup \delta \circ j^{*}\left(p_{I}\right)=\delta(\chi) \cup p_{I} .
$$

Proof of Lemma 101 for odd $\boldsymbol{k}$ Trivial computation shows that the rank of the $\bar{E}_{1}^{*, *}$ is the same as that of $H^{*} B S O(k+1)$. Hence all the differentials vanish.

\subsection{The spectral sequence for the Kazarian space $\mathcal{K}_{\text {Morin }}(k)$ of arbitrary Morin maps}

Recall that $\xi_{r}$ is the universal normal bundle in the source of the stratum of $\Sigma^{1_{r}}$ points, and $\bar{\xi}_{r}$ is that for prim maps. Let $U_{r}$ and $\bar{U}_{r}$ denote their Thom classes, $\operatorname{dim} U_{r}=\operatorname{dim} \bar{U}_{r}=r(k+1)$. Further recall that $A=\mathbb{Q}\left[p_{1}, \ldots, p_{\ell}\right]$ is the subring of $H^{*}(B S O ; \mathbb{Q})$ generated by the first $\ell$ Pontrjagin classes, and $\chi \in H^{2 \ell}(B S O(2 \ell) ; \mathbb{Q})$ is the Euler class.

\section{Lemma 104}

a) For $k=2 \ell$ the group $H^{*}\left(T \xi_{r} ; \mathbb{Q}\right)$ is the following:

$$
H^{*}\left(T \xi_{r} ; \mathbb{Q}\right)=\left\{\begin{array}{ll}
U_{r} \cup A, & \text { if } r \text { is odd, } \\
U_{r} \cup \chi \cdot A, & \text { if } r \text { is even, }
\end{array} \quad r \geq 1 .\right.
$$

where $A=\mathbb{Q}\left[p_{1}, \ldots, p_{\ell}\right]$.

b) For $k=2 \ell+1$ this group is:

$$
H^{*}\left(T \xi_{r} ; \mathbb{Q}\right)= \begin{cases}0, & \text { if } r \text { is odd, } \\ U_{r} \cup A, & \text { if } r \text { is even. }\end{cases}
$$

Proof This Lemma has been essentially proved in [29]. Here we recall the main steps. The maximal compact subgroup $G_{r}^{O}$ of the automorphism group Aut $\left(\eta_{r}\right)$, where $\eta_{r}:\left(\mathbb{R}^{r(k+1)}, 0\right) \rightarrow\left(\mathbb{R}^{(r+1) k+r}, 0\right)$ has isolated $\Sigma^{1_{r}}$ singular point at the origin is $G_{r}^{O}=O(1) \times O(k)$ and its representation $\lambda$ in the source is

$$
\left\lceil\frac{r-1}{2}\right\rceil \cdot 1+\left\lfloor\frac{r+1}{2}\right\rfloor \cdot \varrho_{1}+\left\lceil\frac{r}{2}\right\rceil\left(\varrho_{1} \otimes \varrho_{k}\right)+\left\lfloor\frac{r}{2}\right\rfloor \cdot \varrho_{k}
$$

( $\varrho_{i}$ is the standard representation of $O(i)$ on $\mathbb{R}^{i}$ ).

The "oriented" subgroup of $G_{r}^{O}$ (ie the subgroup that occurs as structure group of the normal bundles of the stratum $\Sigma^{1_{r}}$ of maps from an oriented manifold into an oriented one) is

$$
G_{r}=\left\{(\varepsilon, A) \in O(1) \times O(k) \mid \varepsilon^{r} \cdot \operatorname{det} A>0\right\}
$$


and the group $\bar{G}_{r}$ corresponding to "oriented" prim maps is $\bar{G}_{r}=\{(\varepsilon, A) \mid \varepsilon=1$ and det $A>0\}$. The representations of $\bar{G}_{r}$ and $G_{r}$ are obtained by restricting $\lambda$ to $S O(1) \oplus S O(k)=\bar{G}_{r}$ and to $G_{r}$, respectively. Let us denote these representations by $\bar{\lambda} S O$ and $\lambda^{S O}$. From now on we omit the subindex $r$ in the notation of the groups.

The universal bundle associated to the representation $\bar{\lambda} S O$ of $\bar{G}$ is

$$
\left\lceil\frac{r-1}{2}\right\rceil \cdot 1 \oplus\left\lfloor\frac{r+1}{2}\right\rfloor \gamma_{1}^{S O} \oplus\left\lceil\frac{r}{2}\right\rceil\left(\gamma_{1}^{S O} \otimes \gamma_{k}^{S O}\right) \oplus\left\lfloor\frac{r}{2}\right\rfloor \gamma_{k}^{S O} .
$$

(Note that here both 1 and $\gamma_{1}^{S O}$ denote the trivial line bundle. They are denoted differently because the above mentioned $\mathbb{Z}_{2}$-action, (induced by changing the orientation of the kernel bundle; see Remark 98) changes the orientation of $\gamma_{1}^{S O}$, while keeps it on 1.)

The short exact sequence $1 \rightarrow \bar{G} \rightarrow G \rightarrow \mathbb{Z}_{2} \rightarrow 1$ induces double covers $B \bar{G} \rightarrow B G$ of the universal base spaces and the bundles $\bar{\xi}_{r}$ and $\xi_{r}$ :

$$
\bar{\xi}_{r}=\mathbb{R}^{(r+1) k} \underset{\bar{\lambda} S O}{\times} E \bar{G} \rightarrow \xi_{r}=\mathbb{R}^{(r+1) k} \underset{\lambda S O}{\times} E G .
$$

The action of the corresponding involution on $H^{*}\left(T \bar{\xi}_{r} ; \mathbb{Q}\right)$ is what we want to understand, because $\bar{E}_{1}^{r, *}=H^{*}\left(T \bar{\xi}_{r} ; \mathbb{Q}\right)$ is the $E_{1}$-term of the spectral sequence of the Kazarian space of oriented prim maps $\overline{\mathcal{K}}_{r}$, and its $\mathbb{Z}_{2}$-invariant part is $H^{*}\left(T \xi_{r} ; \mathbb{Q}\right)$ and that is the $E_{1}$ term for the Kazarian space of oriented arbitrary Morin maps.

a) Let first $k$ be even, $k=2 \ell$. Then

$$
H^{*}\left(T \bar{\xi}_{r} ; \mathbb{Q}\right)=\bar{U}_{r} \cup H^{*}(B S O(k) ; \mathbb{Q})=\bar{U}_{r} \cup \mathbb{Q}\left[p_{1}, \ldots, p_{\ell-1}, \chi\right], \operatorname{dim} \chi=k .
$$

The orientation of the fibre will change under this involution by $(-1)^{\lfloor(r+1) / 2\rfloor}$. $(-1)^{\lceil r / 2\rceil}=(-1)^{r+1}$. (The third summand in (9) does not change its orientation since $\varepsilon \cdot \operatorname{det} A>0$, so $\gamma_{1}^{S O}$ and $\gamma_{k}^{S O}$ change or keep their orientations at the same time.) Hence the Thom class $\bar{U}_{r}$ of $\bar{\xi}_{r}$ is mapped into $(-1)^{r+1} \bar{U}_{r}$, and $\chi$ is mapped into $-\chi$. Thus we proved part a).

b) The proof of part b) is similar.

Proof of Lemma 96 The previous Lemma gives us the $E_{1}$ term of the spectral sequence for the Kazarian space of arbitrary Morin maps.

a) Let $k=2 \ell$ and $A=\mathbb{Q}\left[p_{1}, \ldots, p_{\ell}\right]$. Then identifying $U_{r}$ with $\chi^{r}$ we obtain that the $E_{1}$-term looks as follows:

\begin{tabular}{c|c|c|c|c|c|c}
$r$ & 0 & 1 & 2 & 3 & 4 & 5 \\
\hline$E_{1}^{r, *}$ & $A \oplus \chi A$ & $\chi A$ & $\chi^{3} A$ & $\chi^{3} A$ & $\chi^{5} A$ & $\chi^{5} A$
\end{tabular}


The differentials map $\chi^{r} \cdot A$ onto $\chi^{r+1} \cdot A$ in the next column. This follows from Lemma 103. Hence $E_{2}^{0, *}=E_{\infty}^{0, *}=A, E_{2}^{r, *}=E_{\infty}^{r, *}=0$ for $r>0$. We conclude that the composition map

$$
B O(k) \rightarrow B S O(k+1)=\mathcal{K}_{\text {prim }}(k) \rightarrow \mathcal{K}_{\text {Morin }}(k)
$$

induces an isomorphism of the rational cohomology rings. (Here the map $B O(k) \rightarrow$ $B S O(k+1)$ induces the direct sum of the universal unoriented $k$-bundle with its determinant line bundle.)

Hence $\mathcal{K}_{\text {Morin }}(k)$ and $B O(k)$ are rationally homotopically equivalent.

b) For $k=2 \ell+1$

$$
E_{1}^{r, j}=E_{\infty}^{r, j}= \begin{cases}0, & \text { if } r \text { is odd }, \\ p_{\ell+1}^{i} \cup H^{j}(B S O(k) ; \mathbb{Q}), & \text { if } r=2 i .\end{cases}
$$

where $p_{\ell+1}$ is the $\ell+1-$ th Pontrjagin class of the virtual normal bundle. Therefore $H^{*}\left(\mathcal{K}_{\text {Morin }}(k) ; \mathbb{Q}\right) \approx H^{*}(B O(k+1) ; \mathbb{Q})$. This isomorphism of cohomology groups can be induced by the following map. Consider the map

$$
B O(k+1) \rightarrow B S O(k+1)
$$

corresponding to the oriented $k+1$-bundle over $B O(k+1)$ equal to the tensor product of the universal (unoriented) $k+1$-bundle with its determinant line bundle. Then compose it with the composition map

$$
B S O(k+1) \cong \mathcal{K}_{\text {prim }}(k) \rightarrow \mathcal{K}_{\text {Morin }}(k) .
$$

Hence the spaces $\mathcal{K}_{\text {Morin }}(k)$ and $B O(k+1)$ are rationally homotopically equivalent.

Remark 105 The natural forgetting map

$$
\operatorname{Cob}_{\text {prim }}(n, k) \otimes \mathbb{Q} \rightarrow \operatorname{Cob}_{\text {Morin }}(n, k) \otimes \mathbb{Q}
$$

induced by considering an oriented prim map just as an oriented Morin map, is an epimorphism. Indeed, this is equivalent to the claim that $H^{*}\left(\mathcal{K}_{\text {Morin }}(k) ; \mathbb{Q}\right) \rightarrow$ $H^{*}\left(\mathcal{K}_{\text {prim }}(k) ; \mathbb{Q}\right)$ is injective, and this follows from Lemma 99.

\section{Thom polynomials of Morin singularities for Morin maps}

Having computed the rational Kazarian spectral sequence for all Morin maps we have determined all the (higher) Thom polynomials of all $\Sigma^{1_{i}}$-singularities for Morin maps. First we recall the definitions. 
Definition 106 Let $f: M^{n} \rightarrow P^{n+k}$ be a singular map and let $[\eta]$ be a singularity class, let $\eta(f)$ denote the set of $\eta$-points of $f$. Suppose that $\eta(f)$ is a manifold and its closure $\bar{\eta}(f)$ represents a rational homology class dual to a cohomology class $t$ of $M^{n}$.

It has been shown by Thom that for generic $f$ the class $t$ is a characteristic class of the virtual normal bundle of $f$. This characteristic class (expressed as a polynomial of the basic characteristic classes, in our case of the Pontrjagin classes) is called the Thom polynomial of $\eta$ and denoted by $t=T p_{\eta}(f)$.

We define the higher Thom polynomials as follows: Let $\eta$ be a highest singularity of the $\tau$-map $f$. Recall that the normal bundle of $\eta(f)$ in $M^{n}$ can be induced by a homotopically unique map $v_{\eta}: \eta(f) \longrightarrow B G_{\eta}$ from the universal bundle over $B G_{\eta}$. Let $x \in H^{*}\left(B G_{\eta} ; \mathbb{Q}\right)$ be a cohomology class. Let $i: \eta(f) \subset M^{n}$ be the inclusion. Now we can ask how to express the pushforward class $i_{!}\left(v_{\eta}^{*}(x)\right)$ as a polynomial of the characteristic classes of $M^{n}$ and $f^{*}\left(T P^{n+k}\right)$. This expression will be called the higher Thom polynomial corresponding to the normal $G_{\eta}$-characteristic class $x$ of the singularity $\eta$. Hence the set of all higher Thom polynomials associated to $\eta$ can be identified with the image of the map $H^{*}\left(\mathcal{K}_{\tau}, \mathcal{K}_{\tau^{\prime}}\right) \rightarrow H^{*}\left(\mathcal{K}_{\tau}\right)$, where $\tau^{\prime}=\tau \backslash\{\eta\}$. Now for a highest singularity $\eta^{\prime}$ in $\tau^{\prime}$ one can define the higher Thom polynomials as above in $H^{*}\left(\mathcal{K}_{\tau^{\prime}}\right)$. But these are those for $\tau^{\prime}$-maps. If we want to define them for $\tau$-maps, then we have to consider their preimages in $H^{*}\left(\mathcal{K}_{\tau}\right)$, which are well defined modulo the image of $H^{*}\left(\mathcal{K}_{\tau}, \mathcal{K}_{\tau^{\prime}}\right)$. Hence the higher Thom polynomials of the lower singularities are defined modulo those of the higher singularities. Namely Kazarian has shown that the $E_{\infty}$-terms of the cohomological spectral sequence of the Kazarian space $\mathcal{K}_{\tau}$ can be identified with the higher Thom polynomials of the singularities.

Below we show for each element of the $E_{\infty}$ term of the spectral sequence of the Kazarian space for Morin maps $\mathcal{K}_{\text {Morin }}$ a singularity $\eta$ and its normal $G_{\eta}$-characteristic class representing the given element. We start with the simpler case of prim maps.

A) Prim maps: Let $f$ be the composition $f: M^{n} \stackrel{g}{\rightarrow} \mathbb{R}^{n+k+1} \stackrel{\pi}{\longrightarrow} \mathbb{R}^{n+k}$, where $g$ is an immersion. Let $\chi=\chi_{k+1}$ be the Euler class in $H^{k+1}(B S O(k+1) ; \mathbb{Q})$.

1) $k$ odd $=2 \ell+1$. Then $H^{*}(B S O(k) ; \mathbb{Q})=\mathbb{Q}\left[p_{1}, \ldots, p_{\ell}\right]$, recall that we denoted this ring by $A$. The Kazarian space $\mathcal{K}_{\text {prim }}(k)$ for prim maps of codimension $k$, has the same rational homotopy type as $B S O(k+1)$. The Kazarian map $\kappa_{f}: M^{n} \rightarrow \mathcal{K}_{\text {prim }}(k)$ corresponding to the prim map $\mathrm{f}$ can be identified with the normal map of $g$, ie $\kappa_{f}=v_{g}: M^{n} \rightarrow B S O(k+1)$. The cohomology ring of $\mathcal{K}_{\text {prim }}(k)$ is the polynomial ring over $A$ with variable $\chi$, ie $H^{*}(B S O(k+1), Q)=A[\chi]$, where $\chi \in H^{k+1}(B S O(k+1) ; Q)$ is the Euler 
class. The Kazarian spectral sequence degenerates, ie for any $r$ the member $E_{r}^{*, *}$ looks as follows:

\begin{tabular}{c|c|c|c|c|l}
$i=$ & 0 & 1 & 2 & 3 & \\
\hline$E_{r}^{i, *}$ & $A$ & $\chi A$ & $\chi^{2} A$ & $\chi^{3} A$ & \\
\hline$\eta$ & $\Sigma^{0}$ & $\Sigma^{1,0}$ & $\Sigma^{1,1}$ & $\Sigma^{1,1,1}$ &
\end{tabular}

The last row in this table shows the singularity stratum, and the middle raw shows which part of the cohomology ring $H^{*}\left(\mathcal{K}_{\text {prim }}, Q\right)$ is represented by the Thom polynomial and the higher Thom polynomials of this singularity stratum $\eta$ (modulo those of the higher singularities). The minimal dimensional parts of each column give the Thom polynomial of the stratum. Hence $\eta=\Sigma^{1_{i}}(f)$ represents $\chi^{i}$ in $H^{*}\left(M^{n}\right) .{ }^{12}$

Let $x=p_{I}=p_{i_{1}} \ldots p_{i_{S}}, i_{1} \leq \cdots \leq i_{s} \leq \ell$ be a normal characteristic class of $\Sigma^{1_{i}}(f)^{13}$ and let $j_{i}: \Sigma^{1_{i}}(f) \subset M^{n}$ be the embedding. Then $j_{i !}(x)=\chi^{i} \cdot p_{I}$.

2) $k$ even $=2 \ell$. Let us denote again by $A$ the ring $\mathbb{Q}\left[p_{1}, \ldots, p_{\ell}\right]$ and by $\chi=\chi_{k}$ the Euler class $\chi \in H^{k}(B S O(k), Q)$. Then $H^{*}(B S O(k))=A \oplus \chi \cdot A$.

We have seen in Lemma 101 that the $E_{\infty}^{*, *}$ member of the spectral sequence is

\begin{tabular}{c|c|c|c|c|l}
$i$ & 0 & 1 & 2 & 3 & \\
\hline$E_{\infty}^{i, *}$ & $A$ & 0 & 0 & 0 & \\
\hline$\eta$ & $\Sigma^{0}$ & $\Sigma^{1,0}$ & $\Sigma^{1,1}$ & $\Sigma^{1,1,1}$ &
\end{tabular}

The last row shows the singularities corresponding to the blocks (by gluing) of the Kazarian space and hence they correspond to the columns of the Kazarian spectral sequence.

Conclusion All the singularity strata and all their normal characteristic cycles are null-homologous for a prim map of even codimension (except those of the regular stratum $\Sigma^{0}$.)

\section{B) Arbitrary Morin maps:}

1) Case $k$ even $=2 \ell$. The above Conclusion remains true, ie all (higher) Thom polynomials of the strata $\Sigma^{1_{i}}$, for $i \geq 1$ vanish. This follows from Lemma 99.

${ }^{12}$ A priori we obtain only that the Thom polynomial of $\Sigma^{1_{i}}$ is $c \cdot \chi^{i}$, where $c$ is a nonzero rational number. But the similar spectral sequence can be considered with any coefficient ring, containing $1 / 2$. Then we obtain, that for integer coefficients the Thom polynomial of $\Sigma^{1_{i}}$ is equal to $\chi^{i}$, modulo 2 -primary torsion elements.

${ }^{13}$ Note that these Pontrjagin classes are not those of the normal bundle of the stratum. For each singularity $\eta$ here $G_{\eta}=S O(k)$, and $p_{i}$ is the Pontrjagin class in $H^{*}(B S O(k))$. But the normal bundle of the stratum $\Sigma^{1_{i}}$ is $i\left(\gamma_{k}^{S O} \oplus \varepsilon^{1}\right)$. 
2) Case $k$ odd $=2 \ell+1$. The $E_{r}^{*, *}$ members of the spectral sequence are for any $r$ the following. (Recall that $U_{i}$ denotes the Thom class of the normal bundle $\xi_{i}$ of the stratum $\Sigma^{1_{i}}$, it has dimension $i(k+1)$.)

\begin{tabular}{c|c|c|c|c|c|c}
$i$ & 0 & 1 & 2 & 3 & 4 & \\
\hline$E_{r}^{i, *}$ & $A$ & 0 & $U_{2} \cup A$ & 0 & $U_{4} \cup A$ & \\
\hline$\eta$ & $\Sigma^{0}$ & $\Sigma^{1,0}$ & $\Sigma^{1,1}$ & $\Sigma^{1,1,1}$ & $\Sigma^{14}$ &
\end{tabular}

(This is so for the groups $E_{1}^{i, *}$ by Lemma 104, and all the differentials vanish by dimensional reason, since $A$ has no nonzero element of odd degree.)

The higher Pontrjagin classes vanish: $p_{j}\left(v_{f}\right)=0$ for $j>\ell+1$; see Lemma 107 below. The cohomology class in $H^{*}\left(M^{n} ; \mathbb{Q}\right)$ Poincarè dual to the homology class $\left[\bar{\Sigma}^{1}{ }^{i}\right]$ represented by the closure of the singularity stratum $\Sigma^{1_{i}}$ is

$$
D_{M}\left[\bar{\Sigma}^{1_{i}}(f)\right]= \begin{cases}0, & \text { if } i \text { is odd } \\ p_{\ell+1}^{i / 2}, & \text { if } i \text { is even. }\end{cases}
$$

(Note that $\left.H^{*}\left(B G_{\Sigma^{1}{ }_{2 i}}\right)=H^{*}(B S O(2 \ell+1))=\mathbb{Q}\left[p_{1}, \ldots, p_{\ell}\right]\right)$.

If $x \in H^{*}\left(B G_{\Sigma^{1} 1_{2 i}} ; \mathbb{Q}\right)$ is a monomial on the Pontrjagin classes $x=p_{I}=$ $p_{i_{1}} \ldots p_{i_{s}}, i_{1} \leq \cdots \leq i_{s} \leq \ell$, then $\left(j_{2 i}\right)_{!}\left(p_{I}\right)=p_{\ell+1}^{i} \cdot p_{I} \in H^{*}\left(M^{n}\right)$. Hence any characteristic class of the normal bundle $v_{f}$ of the map $f$ is represented by a higher Thom polynomial.

Lemma 107 If $f: M^{n} \rightarrow P^{n+k}$ is a Morin map, the manifolds $M^{n}$ and $P^{n+k}$ are oriented, the codimension $k$ is odd, $k=2 \ell+1$ and $v_{f}: M^{n} \rightarrow B S O$ is the virtual normal bundle, then $p_{j}\left(v_{f}\right)=0$ for $j>\ell+1$.

Proof Since $f$ is a Morin map, the stable normal map $v_{f}: M^{n} \rightarrow B S O$ can be decomposed as $v_{f}=\pi \circ \kappa_{f}$, where $\kappa_{f}: M^{n} \rightarrow \mathcal{K}_{\text {Morin }}(k)$ is the map into the Kazarian space.

Now it remained to show that $\pi^{*}\left(p_{j}\right)=0$ for $j>\ell+1$.

This follows from the diagram

$$
\begin{aligned}
& H^{*}(B S O(2 \ell+2))=H^{*}\left(\mathcal{K}_{\text {prim }}\right) \stackrel{p^{*}}{\longleftrightarrow} H^{*}\left(\mathcal{K}_{\text {Morin }}(k)\right) \\
& \uparrow_{\bar{\pi}^{*}} \uparrow_{\pi^{*}} \\
& H^{*}(B S O)=H^{*}(B S O)
\end{aligned}
$$

Here $p: \mathcal{K}_{\text {prim }}(k) \rightarrow \mathcal{K}_{\text {Morin }}(k)$ is the quotient map. Hence $p^{*}$ is injective. Now we see that $\bar{\pi}^{*}\left(p_{j}\right)=0$ for $j>\ell+1$, hence $\pi^{*}\left(p_{j}\right)=0$. 


\section{$15 \quad \Sigma^{1_{r}}-$ maps}

Let us denote by $\operatorname{Cob}_{\Sigma^{1 r}}(n, k)$ the oriented cobordism group of Morin maps having at most $\Sigma^{1^{1}}$ singularities. Recall that $A=\mathbb{Q}\left[p_{1}, \ldots, p_{\ell}\right]$.

Theorem 7 The group $\operatorname{Cob}_{\Sigma^{1 r}}(n, k) \otimes \mathbb{Q}$ is isomorphic to the degree $n$ part of the following graded ring:

$$
\begin{cases}A, & \text { if } k=2 \ell \text { and } r \text { is odd, } \\ A \oplus \chi^{r+1} \cdot A, & \text { if } k=2 \ell \text { and } r \text { is even, } \\ A / p_{\ell}^{[(r+1) / 2]}=0, & \text { if } k=2 \ell-1 .\end{cases}
$$

Proof We have to consider the above Kazarian spectral sequence restricted to the first $r+1$ columns.

\section{Elimination of singularities}

Arnold and his coauthors in [4, page 212] posed the question whether vanishing of the Thom polynomial of a singularity $\eta$ for a map $f$ is enough for having a map $g$ homotopic to $f$ and having no $\eta$ points.

The answer to this question is negative [40]. Here we consider the analogous question of elimination by $\tau$-cobordism and give a complete solution to it.

Let $\tau$ be as before a set of stable singularities. Let $\eta$ be a top singularity in $\tau$. Let $f: M^{n} \rightarrow P^{n+k}$ be a $\tau$-map. Then clearly the restriction of $f$ to the $\eta$-stratum $\eta(f)$ is an immersion and its normal bundle is induced from the bundle $\widetilde{\xi}_{\eta}$. We show that the cobordism class of this immersion $\left.f\right|_{\eta}(f)$ with normal bundle induced from $\widetilde{\xi}_{\eta}$ is a complete (necessary and sufficient) obstruction to the elimination of $\eta$-points by a $\tau$-cobordism.

Translating this obstruction into cohomologies we obtain the following: Suppose that the Gysin map $f$ ! annihilates not only the Thom polynomial of $\eta$ for the map $f$, but also all its higher Thom polynomials of $\eta$ (ie the images of the characteristic classes of the normal bundle of $\eta(f) \subset M^{n}$ under the Gysin map induced by this inclusion. Then a nonzero multiple of the $\tau$-cobordism class of $f$ contains an $\eta$-free map.

Notation 108 Given a vector bundle $\zeta$ over a given base space and a smooth manifold $P^{n+k}$, we will denote by $\operatorname{Imm}^{\xi}\left(P^{n+k}\right)$ the cobordism group of immersions in $P^{n+k}$ with normal bundle induced from $\zeta$. 
Theorem 8 Let $f: M^{n} \rightarrow P^{n+k}$ be a $\tau-$ map, $\tau=\left\{\eta_{0}<\eta_{1}<\cdots<\eta_{i}\right\}$ and $\tau^{\prime}=$ $\left\{\eta_{0}<\cdots<\eta_{i-1}\right\}$. Recall that $\left.f\right|_{\eta_{i}(f)}: \eta_{i}(f) \rightarrow P^{n+k}$ is an immersion with normal bundle induced from the universal bundle $\widetilde{\xi}_{i}=E G_{i} \times \widetilde{\lambda}_{i} \mathbb{R}^{c_{i}+k}$. Hence it represents an element $\theta(\eta, f) \in \operatorname{Imm}^{\widetilde{\xi}_{i}}\left(P^{n+k}\right)$ in the cobordism group of $\widetilde{\xi}_{i}$-immersions. We claim that this element $\theta(\eta, f)$ vanishes if and only if there exists a $\tau^{\prime}-$ map $g: M^{n} \rightarrow P^{n+k}$ $\tau$-cobordant to $f$.

Proof Consider the cofibration

$$
V_{i-1} \subset V_{i} \rightarrow V_{i} / V_{i-1} \rightarrow T \tilde{\xi}_{\eta}
$$

of virtual complexes, and apply the functor $\Gamma=\Omega^{\infty} S^{\infty}$. (Let us ignore for a while the fact that $V_{i}$ and $V_{i-1}$ are virtual complexes, and let us treat them at first if they were usual complexes.)

By a theorem of [6] the functor $\Gamma$ turns a cofibration into a fibration. Hence we obtain a fibration

$$
X_{\tau^{\prime}} \subset X_{\tau} \rightarrow \Gamma T \widetilde{\xi}_{i}
$$

Applying the homotopy functor $\left[P^{n+k},-\right]$ to this fibration, we obtain that the sequence

$$
\operatorname{Cob}_{\tau^{\prime}}\left(P^{n+k}\right) \longrightarrow \operatorname{Cob}_{\tau}\left(P^{n+k}\right) \stackrel{\beta}{\longrightarrow} \operatorname{Imm}^{\tilde{\xi}_{i}}\left(P^{n+k}\right)
$$

is exact. $\beta$ maps $[f]$ to $\theta(\eta, f)$.

Now let us address the problem that $V_{i}$ and $V_{i-1}$ are virtual complexes. For any natural number $m$ there exist: a finite dimensional approximation $V_{i \text {, fin }}$ and a natural number $N$ such that the suspension $S^{N} V_{i \text {,fin }}$ exists and it represents the $N+m$-homotopy type of $S^{N} V_{i}$.

Similarly we can suppose that for the same $m$ and $N$ the same holds for $i-1$ substituted for $i$, and $S^{N} V_{i \text {,fin }}=S^{N} V_{i-1 \text {,fin }} \cup D^{N} \times T \widetilde{\xi}_{\eta \text {,fin }}$ for a finite approximation $T \widetilde{\xi}_{\eta \text {,fin }}$ of the Thom space $T \widetilde{\xi}_{\eta}$ ( $m$-equivalent to it). Now instead of the virtual cofibration above we consider the (usual) cofibration

$$
S^{N} V_{i-1, \text { fin }} \subset S^{N} V_{i, \text { fin }} \rightarrow S^{N} T \tilde{\xi}_{\eta, \text { fin }}
$$

and apply the functor $\Gamma$ to it. We obtain a fibration

$$
\Gamma S^{N} V_{i-1, \text { fin }} \rightarrow \Gamma S^{N} V_{i, \text { fin }} \rightarrow \Gamma S^{N} T \widetilde{\xi}_{\eta, \text { fin }}
$$

(here the first space is the fibre the second is the total space and the third one is the base space). 
Let us recall that whenever we have such a sequence of maps $F \rightarrow E \rightarrow B$ (where $F$ is the fibre of the fibration $E \rightarrow B$ ) we can continue it to the left infinitely and obtain an infinite to the left sequence of fibrations (called the resolvent of the map $E \rightarrow B$ ):

$$
\ldots \Omega^{2} F \rightarrow \Omega^{2} E \rightarrow \Omega^{2} B \rightarrow \Omega F \rightarrow \Omega E \rightarrow \Omega B \rightarrow F \rightarrow E \rightarrow B
$$

In particular we can consider the fibration of $N$-th loop spaces

$$
\Omega^{N} \Gamma S^{N} V_{i-1, \text { fin }} \rightarrow \Omega^{N} \Gamma S^{N} V_{i, \text { fin }} \rightarrow \Omega^{N} \Gamma S^{N} T \widetilde{\xi}_{\eta, f i n} .
$$

Noticing the $\Omega^{N} \Gamma S^{N}=\Gamma$, we obtain a usual fibration $m$-equivalent to the fibration obtained from the cofibration of virtual complexes.

Definition 109 The fibre bundle (10) will be called the key fibration for the pair $\left(\tau, \tau^{\prime}\right)$.

Remark 110 The existence of such a fibre bundle was conjectured by Endre Szabó. This observation and the Kazarian conjecture are the main points of the paper.

Corollary $111[f] \otimes 1_{\mathbb{Q}} \in \operatorname{Cob}_{\tau}\left(P^{n+k}\right) \otimes \mathbb{Q}$ contains a $\tau^{\prime}-$ map $g($ ie $\eta(g)=\varnothing)$ iff for any $x \in H^{*}\left(B G_{\eta} ; \mathbb{Q}\right)$ the class $\left(\left.f\right|_{\eta(f)}\right) !\left(j^{*}(x)\right)=0$ in $H^{*}\left(P^{n+k} ; \mathbb{Q}\right)$, where $j: \eta(f) \rightarrow B G_{\eta}$ is the map inducing the normal bundle of $\eta(f)$ in $M^{n}$.

Proof Let $\theta_{f} \in\left\{P^{n+k}, T \widetilde{\xi}_{\eta}\right\}$ be the stable homotopy class corresponding to the cobordism class $\theta(\eta, f)$ of the immersion $\left.f\right|_{\eta(f)}: \eta(f) \rightarrow P^{n+k}$. It is standard to show (as in [40]) that $\theta_{f}^{*}(U \cup x)=(f \mid \eta(f)) !\left(j^{*}(x)\right)$, where $U \in H^{*}\left(T \widetilde{\xi}_{\eta} ; \mathbb{Q}\right)$ is the Thom class. The map

$$
\begin{aligned}
\operatorname{Imm} \tilde{\xi}_{\eta}\left(P^{n+k}\right) \otimes \mathbb{Q}=\left\{P^{n+k}, T \tilde{\xi}_{\eta}\right\} \otimes \mathbb{Q} & \longrightarrow \operatorname{Hom}\left(H^{*}\left(T \widetilde{\xi}_{\eta} ; \mathbb{Q}\right), H^{*}\left(P^{n+k} ; \mathbb{Q}\right)\right) \\
\alpha & \longmapsto \alpha^{*}
\end{aligned}
$$

is an isomorphism (see for example [40, page 321]). Hence the class $\left[\left.f\right|_{\eta(f)}\right] \otimes 1_{\mathbb{Q}} \in$ $\operatorname{Imm}^{\widetilde{\xi}_{n}}\left(P^{n+k}\right) \otimes \mathbb{Q}$ is equal to $\theta_{f} \otimes 1_{\mathbb{Q}} \in\left\{P^{n+k}, T \widetilde{\xi}_{\eta}\right\}$ and that corresponds to the $\operatorname{map} \theta_{f}^{*}$.

Remark 112 This Corollary shows that while the answer was negative to the question of Arnold about the elimination of the highest singularity by homotopy - vanishing of the corresponding Thom polynomial is not enough for homotopical elimination - the reformulated question about elimination by $\tau$-cobordism of the top singularity $\eta$ of a $\tau$-map has an answer quite close to the one formulated in [4]. Namely after taking the tensor product with the rational numbers $\mathbb{Q}$ of the cobordism group of $\tau$-maps into a fixed manifold $P^{n+k}$ we obtain, that an element $\left[f: M^{n} \rightarrow P^{n+k}\right]$ of this group contains an $\eta$-free map iff all the characteristic numbers of $\tau$-maps "coming from $\eta$ " 
vanish for $f$, ie all the $(x, z)$-characteristic numbers $\left\langle\kappa_{f}^{*}(x) \cup f^{*}(z),\left[M^{n}\right]\right\rangle$ vanish if $x \in \operatorname{im}\left(H^{*}\left(\mathcal{K}_{\tau}, \mathcal{K}_{\tau}^{\prime}, \mathbb{Q}\right) \rightarrow H^{*}\left(\mathcal{K}_{\tau} ; \mathbb{Q}\right)\right)$; see Definition 92 .

\section{Part VI General theorems on the key fibration $X_{\tau} \stackrel{X_{\tau^{\prime}}}{\longrightarrow} \Gamma T \tilde{\xi}_{\eta}$}

Here we shall study this bundle from rational point of view and give a general structure theorem for it. There are quite general conditions implying that this bundle is a direct product rationally. In this case for any oriented manifold $P^{n+k}$

$$
\operatorname{Cob}_{\tau}\left(P^{n+k}\right) \otimes \mathbb{Q} \approx\left(\operatorname{Cob}_{\tau^{\prime}}\left(P^{n+k}\right) \otimes \mathbb{Q}\right) \oplus\left(\operatorname{Imm}^{\tilde{\xi}_{\eta}}\left(P^{n+k}\right) \otimes \mathbb{Q}\right) .
$$

Definition 113 We say that a fibration $\pi: E \stackrel{F}{\longrightarrow} B$ is an $H$-fibration, if all the spaces $E, F, B$ are $H$-spaces of finite types ${ }^{14}$ and the maps $F \subset E \rightarrow B$ are $H-$ homomorphisms.

Definition 114 We say that an $H$-map is $F$-trivial ( $E$-trivial, $B$-trivial respectively) if the space $F$ (respectively $E$ or $B$ ) is contractible.

Lemma $115(\mathbb{Q}$-classification of $H$-bundles) Any $H$-fibration can be represented rationally as a product of three $H$-fibrations, which are $F-, E$ - and $B$-trivial, respectively.

The proof of this Lemma is a standard application of spectral sequences therefore we omit it (see our preprint [41]).

Theorem 9 Let $\eta$ be a top singularity class in $\tau, \tau^{\prime}=\tau \backslash\{\eta\}$. Suppose that the universal bundles $\xi_{\eta}$ and $\widetilde{\xi}_{\eta}$ are oriented and at least one of them has nonzero rational Euler class: $e\left(\xi_{\eta}\right) \neq 0$, or $e\left(\tilde{\xi}_{\eta}\right) \neq 0$ in $H^{*}\left(B G_{\eta} ; \mathbb{Q}\right)$. Then the key fibre bundle

$$
X_{\tau} \stackrel{X_{\tau^{\prime}}}{\longrightarrow} \Gamma T \widetilde{\xi}_{\eta}
$$

splits rationally, ie $X_{\tau} \cong \mathbb{Q}_{\tau^{\prime}} \times \Gamma T \widetilde{\xi}_{\eta}$.

\footnotetext{
${ }^{14}$ ie their homology groups are finitely generated in each dimension.
} 
Proof Let us consider the diagram (the homology groups are meant with rational coefficients):

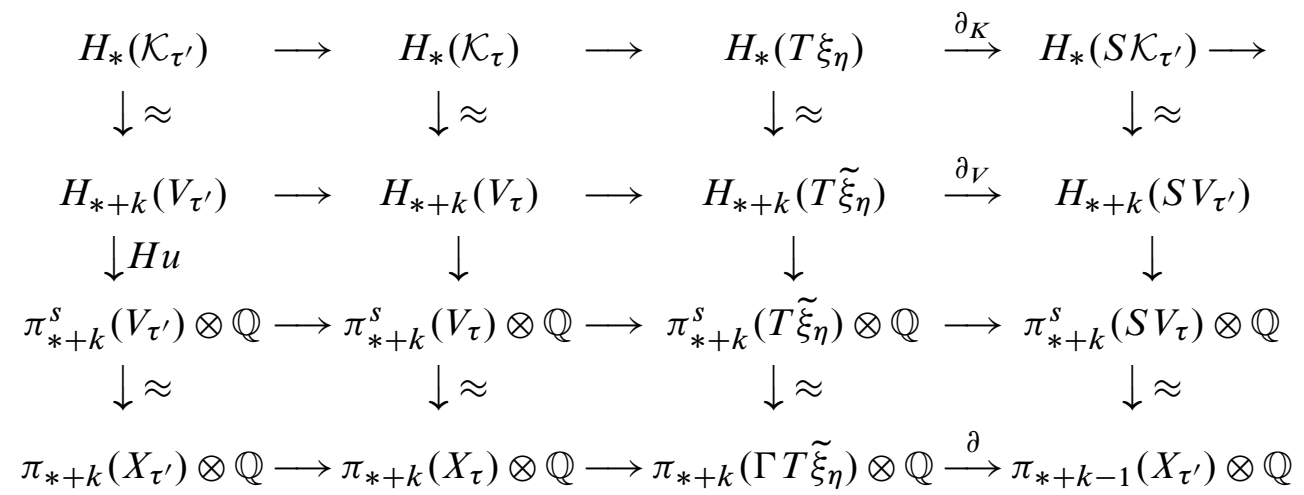

The two upper rows are essentially the homology exact sequences of the pairs $\left(\mathcal{K}_{\tau}, \mathcal{K}_{\tau^{\prime}}\right)$ and $\left(V_{\tau}, V_{\tau^{\prime}}\right)$, respectively. We are writing $H_{*}\left(S \mathcal{K}_{\tau^{\prime}}\right)$ instead of $H_{*-1}\left(\mathcal{K}_{\tau^{\prime}}\right)$, because we consider this row as the dual of the cohomology sequence, which is obtained from the Puppe sequence (see Chapter 9 in [16])

$$
\mathcal{K}_{\tau^{\prime}} \subset \mathcal{K}_{\tau} \rightarrow T \xi_{\eta} \hookrightarrow S \mathcal{K}_{\tau^{\prime}}
$$

applying the functor $[-, K(\mathbb{Q}, *)]$. Then $\partial_{K}$ is the dual of the map induced by the inclusion $j: T \xi_{\eta} \hookrightarrow S \mathcal{K}_{\tau^{\prime}}$.

But the map $j^{*}: H^{*}\left(S \mathcal{K}_{\tau^{\prime}}\right) \rightarrow H^{*}\left(T \widetilde{\xi}_{\eta}\right)$ is trivial if $e\left(\xi_{\eta}\right) \neq 0$. Indeed, the multiplication in $H^{*}\left(S \mathcal{K}_{\tau^{\prime}}\right)$ is trivial, while in $H^{*}\left(T \xi_{\eta}\right)$ any element has the form $U_{\eta} \cup x$ for some $x \in H^{*}\left(B G_{\eta}\right)$ and $\left(U_{\eta} \cup x_{1}\right) \cup\left(U_{\eta} \cup x_{2}\right)=U_{\eta} \cup e\left(\xi_{\eta}\right) \cup x_{1} \cup x_{2} \neq 0$. $\left(H^{*}\left(B G_{\eta} ; \mathbb{Q}\right)\right.$ has no zero divisor since it is a subring of the ring of polynomials $H^{*}(B T ; \mathbb{Q}$,$) where T$ is the maximal torus in $\left.G_{\eta}.\right)$

If $e\left(\widetilde{\xi}_{\eta}\right) \neq 0$, then one can use similarly the second row. (The vertical arrows from the first row are the Thom isomorphisms corresponding to the virtual bundle $v^{k}$, those from the second row are the stable Hurewicz homomorphisms (being isomorphism modulo torsion).) In any of the two cases $\left(e\left(\xi_{\eta}\right) \neq 0\right.$ or $\left.e\left(\widetilde{\xi}_{\eta}\right) \neq 0\right)$ the map $\partial: \pi_{*+k}\left(\Gamma T \widetilde{\xi}_{\eta}\right) \otimes$ $\mathbb{Q} \rightarrow \pi_{*+k-1}\left(X_{\tau^{\prime}}\right) \otimes \mathbb{Q}$ vanishes (see the bottom row). Then $\pi_{*}\left(X_{\tau}\right) \otimes \mathbb{Q}=\pi_{*}\left(X_{\tau^{\prime}}\right) \otimes$ $\mathbb{Q} \oplus \pi_{*}\left(\Gamma T \widetilde{\xi}_{\eta}\right) \otimes \mathbb{Q}$. The spaces $X_{\tau}, X_{\tau^{\prime}}, \Gamma T \widetilde{\xi}_{\eta}$ - being $H$-spaces of finite types - are rationally homotopically equivalent to products of some rational Eilenberg-MacLane spaces $K(\mathbb{Q}, i)$. It follows that $X_{\tau} \cong \mathbb{Q} X_{\tau^{\prime}} \times \Gamma T \widetilde{\xi}_{\eta}$.

Now we give a general rational decomposition theorem of the key bundle $X_{\tau} \stackrel{X_{\tau^{\prime}}}{\longrightarrow} \Gamma T \widetilde{\xi}_{\eta}$. Let $\tau, \tau^{\prime}, \eta$ be as above. Let us denote the rational homotopy types of the spaces $X_{\tau}$, $X_{\tau^{\prime}}, \Gamma T \widetilde{\xi}_{\eta}$ by $X, X^{\prime}$ and $\Gamma$, respectively. Recall that all these spaces are rational 
$H$-spaces, hence they decompose into products of rational Eilenberg-MacLane spaces $K(\mathbb{Q}, i)$ in a unique way.

\section{Theorem 10}

a) There is a rational $H$-space $B$ (ie $B$ is a product of spaces $K(Q, i)$ for some $i)$, such that $X \cong \mathbb{Q}\left(\left(X^{\prime} \times \Omega B\right) / \Omega \Gamma\right) \times B$. (Note that dividing by the space $\Omega \Gamma$ means that its Eilenberg-MacLane factors $K(\mathbb{Q}, j)$ all occur as factors of the numerator and we cancel these factors.)

b) The rational space $B$ is defined in a unique way by the following formula:

$$
\pi_{m}(B)=E_{r, m-r-k}^{\infty} \otimes \mathbb{Q},
$$

where $E_{r, *}^{\infty}$ is the last column (corresponding to the top singularity $\eta=\eta_{r}$ ) in the homological spectral sequence of the Kazarian space $\mathcal{K}_{\tau}$.

Proof By the $\mathbb{Q}$-classification theorem of $H$-bundles we have: There exist $H-$ bundles:

$$
* \stackrel{\Omega A}{\longrightarrow} A, \quad B \stackrel{*}{\longrightarrow} B \quad C \stackrel{C}{\longrightarrow} *
$$

whose product is the bundle $X \longrightarrow \Gamma$ with fibre $X^{\prime}$. In particular, $X=B \times C$; $X^{\prime}=\Omega A \times C$ and $\Gamma=A \times B$ and so $\Omega \Gamma=\Omega A \times \Omega B$.) Part a) is trivial now.

To prove b) note that $\pi_{*}(X)=\pi_{*}(B) \oplus \pi_{*}(C)$ and $\pi_{*}(\Gamma)=\pi_{*}(B) \oplus \pi_{*}(A)$ and $p_{*}: \pi_{*}(X) \rightarrow \pi_{*}(\Gamma)$ is isomorphism on $\pi_{*}(B)$, and this is the maximal such subgroup both in $\pi_{*}(X)$ and in $\pi_{*}(\Gamma)$. There is a commutative diagram

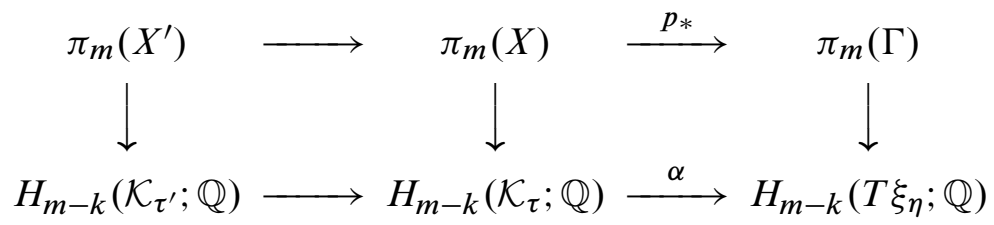

where the vertical arrows are the compositions of a stable Hurewicz homomorphism and a Thom isomorphism. Hence they are isomorphisms. For example the middle vertical arrow is the following:

$$
\begin{gathered}
\left.\pi_{*}(X)=\pi_{*}^{s}\left(V_{\tau}\right) \otimes \mathbb{Q} \stackrel{h^{V_{\tau}}}{\longrightarrow} H_{*}\left(V_{\tau} ; \mathbb{Q}\right)=H_{*}\left(T v^{k} ; \mathbb{Q}\right) \stackrel{\text { Thom }}{\sim} H_{*-k}\left(\mathcal{K}_{\tau} ; \mathbb{Q}\right) .\right) \\
H_{m-k}\left(\mathcal{K}_{\tau} ; \mathbb{Q}\right)=\bigoplus_{i=0}^{r} E_{i, m-k-i}^{\infty} \\
E_{r, m-k-r}^{\infty}=\operatorname{im} \alpha: H_{m-k}\left(\mathcal{K}_{\tau} ; \mathbb{Q}\right) \rightarrow H_{m-k}(\underbrace{T \xi_{\eta}}_{\mathcal{K}_{\tau} / \mathcal{K}_{\tau^{\prime}}} ; \mathbb{Q})
\end{gathered}
$$

Now we have $\pi_{m}(B)=\operatorname{im}\left(p_{*}\right)=\operatorname{im} \alpha=E_{r, m-k-r}^{\infty}$. 
Example 116 Under the conditions of Theorem 9 the equality $B=\Gamma$ holds and so $X=X^{\prime} \times \Gamma$.

\section{A Postnikov-like tower for the classifying space $X_{\tau}$ of $\tau-$ maps}

\section{Theorem 11}

a) The space $X_{\tau}$ classifying cobordisms of $\tau$-maps for $\tau=\left\{\eta_{0}<\eta_{1}<\cdots<\eta_{r}\right\}$ can be obtained by a sequence of fibrations:

$$
\Gamma_{r} \stackrel{\Gamma_{r-1}}{\longleftarrow} A_{2} \stackrel{\Gamma_{r-2}}{\longleftarrow} A_{3} \ldots \stackrel{\Gamma_{1}}{\longleftarrow} X_{\tau}
$$

where $\Gamma_{i}=\Gamma T \widetilde{\xi}_{\eta_{i}}$

b) For any manifold $P^{n+k}$ there is a spectral sequence with $E_{1}$-term

$$
E_{1}^{i, j}=\operatorname{Imm}^{\zeta_{i}}\left(P^{n+k} \times \mathbb{R}^{j}\right),
$$

where $\zeta_{i}=\widetilde{\xi}_{\eta_{i}}$ is the universal normal bundle of the $\eta_{i}$-stratum in the target, and converging to $\operatorname{Cob}_{\tau}\left(P^{n+k} \times \mathbb{R}^{i+j}\right)$.

Recall that given a vector bundle $\zeta$, and a manifold $Q \operatorname{Imm}^{\zeta_{i}}(Q)$ denotes the cobordism group of immersions of closed oriented manifolds into $Q$ with normal bundle induced from $\zeta$.

Proof a) Let $\tau_{j}$ be $\tau_{j}=\left\{\eta_{0}<\eta_{1}<\cdots<\eta_{j}\right\}$ and denote by $V_{j}$ the virtual complex $V_{\tau_{j}}$, and by $\Gamma_{j}$ the space $\Gamma T \widetilde{\xi}_{j}=\Gamma\left(V_{j} / V_{j-1}\right)$. Now put $A_{s} \stackrel{\text { def }}{=} \Gamma\left(V_{r} / V_{r-s}\right)$ and recall that $\Gamma$ turns a cofibration of spaces (or virtual spaces) into fibration.

b) In the same way as the Postnikov tower of a space $X$ induces a spectral sequence starting with $\left[S^{j} P, K(\pi, i)\right]=H^{i-j}\left(P^{n+k}, \pi\right)$ and converging to $\left[P^{n+k}, X\right]$ for any $P^{n+k}$ (see Mosher and Tangora [28]), a Postnikov-like tower induces the spectral sequence in the Theorem.

Recall that $c_{j}$ denotes the codimension of the singularity $\eta_{j}$ in the source. Let us denote by $s_{1}, s_{2}, \ldots, s_{\ell-1}$ those indices, where the parity of the number $c_{j}$ changes, and $s_{\ell}=r$, ie

$$
c_{1} \equiv c_{2} \equiv \cdots \equiv c_{s_{1}} \not \equiv c_{s_{1}+1} \equiv c_{s_{1}+2} \equiv \cdots \equiv c_{s_{2}} \not \equiv c_{s_{2}+1} \equiv \ldots \quad \bmod 2 .
$$

We shall say that the indices $i$ and $i^{\prime}, 1 \leq i<i^{\prime} \leq r$ belong to the same block if $\exists t: s_{t}<i<i^{\prime} \leq s_{t+1}$. 
Let us consider the (homological) Kazarian spectral sequence $E_{i, j}^{1}$ (with rational coefficients) of the space $\mathcal{K}_{\tau}$ (denoted also by $\mathcal{K}_{r}$ ) induced by the filtration $\mathcal{K}_{0} \subset$ $\mathcal{K}_{1} \subset \cdots \subset \mathcal{K}_{r}$, where $\mathcal{K}_{\beta}=\bigcup_{\alpha \leq \beta} D \xi_{\alpha}$ (ie $\mathcal{K}_{\beta}$ is the Kazarian space for the set of singularity classes $\left\{\eta_{0}<\cdots<\eta_{\beta}\right\}$ for $\beta \leq r$.

\section{Proposition 117}

a) A differential $d_{t}: E_{i, j}^{t} \rightarrow E_{i^{\prime}, j^{\prime}}^{t}$ vanishes in this spectral sequence, if the indices $i, i^{\prime}$ belong to the same block.

b) The quotient spaces $\mathcal{K}_{s_{t+1}} / \mathcal{K}_{s_{t}}$ have the same rational homology groups as the wedge products $T \xi_{s_{t}+1} \vee T \xi_{s_{t}+2} \vee \cdots \vee T \xi_{s_{t+1}}$.

c) The space $X_{\tau}$ admits a simplified Postnikov-like tower, containing $\ell$ fibrations ( $\ell=$ the number of the blocks) with fibres

$$
\begin{gathered}
\widehat{\Gamma}_{t}=\prod_{s_{t}<\alpha \leq s_{t+1}} \Gamma T \widetilde{\xi}_{\alpha}, \quad t=1,2, \ldots, \ell . \\
* \longleftarrow \widehat{\Gamma}_{\ell} \stackrel{\widehat{\Gamma}_{\ell-1}}{\longleftarrow} \widehat{A}_{2} \stackrel{\widehat{\Gamma}_{\ell-2}}{\longleftarrow} \widehat{A}_{3} \ldots \stackrel{\widehat{\Gamma}_{1}}{\longleftarrow} X_{\tau} .
\end{gathered}
$$

Proof For any Lie group $G$ the cohomology ring $H^{*}(B G ; \mathbb{Q})$ has only even dimensional elements (since this is the invariant part under the Weyl group action of $H^{*}(B T ; \mathbb{Q})$, where $T$ is the maximal torus in $G$, and $H^{*}(B T ; \mathbb{Q})$ is the tensor product of $\operatorname{dim} T$ copies of the ring $H^{*}\left(C P^{\infty} ; \mathbb{Q}\right)$ ). It follows that $E_{i, j}^{1}=H_{i+j}\left(T \xi_{i} ; \mathbb{Q}\right)$ can be nontrivial only if $i+j \equiv c_{i} \bmod 2$. Hence $E_{i, j}^{r}$ can be nontrivial only if $i+j \equiv c_{i}$ $\bmod 2$. Now $d_{i, j}^{r}: E_{i, j}^{r} \rightarrow E_{i-r, j+r-1}^{r}$ can be nontrivial only if both groups are nontrivial. This cannot be, if they are in the same block.

We have proven a) and from this part b) also follows.

For part c), apply the functor $\Gamma$ to the filtration

$$
V_{s_{1}} \subset V_{s_{2}} \subset \ldots V_{s_{l}}
$$

to obtain the sequence of fibrations in c). The fact that the fibres decompose into products follows from the fact that $V_{s_{t}} / V_{s_{t+1}}$ and the wedge product $T \widetilde{\xi}_{s_{t}+1} \vee \cdots \vee T \widetilde{\xi}_{s_{t+1}}$ are stably rationally homotopically equivalent. Note that the functor $\Gamma$ turns wedge product into product, ie $\Gamma(U \vee V)=\Gamma(U) \times \Gamma(V)$.

Example 118 Consider Morin maps of odd codimension $k$, and having at most $\Sigma^{1^{r}}$ singularities, ie $\tau=\left\{\Sigma^{0}<\Sigma^{1,0}<\Sigma^{1,1,0}<\cdots<\Sigma^{1_{r}, 0}\right\}$.

Then $X_{\tau} \cong \mathbb{Q} \prod_{i \leq r} \Gamma T \widetilde{\xi}_{i}$. For $i$ odd the space $T \xi_{i}$ is rationally trivial (see Lemma 104), hence so is the space $\Gamma T \widetilde{\xi}_{i}$. For $i=2 j$ we have $\Gamma T \widetilde{\xi}_{i} \cong \mathbb{Q} \Gamma S^{i} T(i+1) \gamma_{k}^{S O}$. 
Hence for any $P^{n+k}$ manifold we have

$$
\operatorname{Cob}_{\tau}\left(P^{n+k}\right) \otimes \mathbb{Q} \approx \bigoplus_{2 i \leq r} \operatorname{Imm}^{\tilde{\xi}_{2 i}}\left(P^{n+k}\right) \otimes \mathbb{Q}
$$

where $\widetilde{\xi}_{2 i}$ is the universal normal bundle in the target of the stratum $\Sigma^{1_{2 i}}$.

Example 119 Take for $\tau$ the previous set of singularities for any $r \geq 2$ and add to it as a highest singularity the class of $\eta=I I I_{2,2}$ (see Mather [26]) which is the simplest stable singularity of type $\Sigma^{2,0}$. The universal normal bundle $\widetilde{\xi}_{\eta}$ can be found in [29, Theorem 7].

1) When the codimension $k$ of the maps is odd, then by the previous theorem we have again that the classifying space $X_{\tau}$ splits into the product

$$
X_{\tau}=X_{\tau^{\prime}} \times \Gamma T \tilde{\xi}_{\eta}
$$

where $\tau^{\prime}=\left\{\leq \Sigma^{1}\right\}$, and $X_{\tau^{\prime}}$ splits into product according to the previous example.

2) Equality (11) holds for $k$ even too if $r=\infty$, because in this case the Kazarian space of all (oriented) Morin maps is rationally homotopy equivalent to $B O(k+1)$ and this has only even dimensional rational cohomology groups, and the codimension of the $I I I_{(2,2)}$-stratum is even. (The cohomological exact sequence of the pair $\left(\mathcal{K}_{\tau}, \mathcal{K}_{\tau^{\prime}}\right)$ with rational coefficients splits by dimensional reason.)

Example 120 (Quasi-holomorphic $\tau$-maps) (This example is a bit out of the line of the paper.) Let this time $\tau$ denote a set of holomorphic singularity classes, ie classes of holomorphic germs $\left.\left(C^{*}, 0\right) \rightarrow C^{*+k}, 0\right)$. We call a (noncomplex) smooth map $f$ of a smooth manifold into another one quasi-holomorphic if it imitates the behaviour of holomorphic maps in the following sense:

The source and target manifolds have smooth stratifications such that $f$ maps strata into strata, further there exists a fibrewise diffeomorphism of the tubular neighbourhood of each stratum onto the total space of a complex vector bundle such that the map $f$ gives a fibrewise map from the tubular neighbourhoods of the strata in the source into those in the target, and the arising mappings from fibre to fibre are maps from a complex linear space into another, and all these maps from fibre into fibre belong to the classes in $\tau$. (This time singularity classes are formed using $\mathcal{A}$-equivalence by left-right holomorphic diffeomorphisms.) The cobordism group of quasi holomorphic $\tau$-maps of $n$-manifolds into $P^{n+k}$ can be defined and it will be denoted by $\operatorname{Cob}_{\tau}^{C}\left(P^{n+k}\right)$. 
A classifying space for the cobordisms of quasi holomorphic maps $X_{\tau}^{C}$ can be defined analogously to the real case.

Claim $121 X_{\tau}^{C} \cong \mathbb{Q} \prod \Gamma T \widetilde{\xi}_{i}^{C}$, where $\tilde{\xi}_{i}^{C}$ are the universal complex vector bundles of the strata in the target.

Proof Since each $\xi_{i}$ is a complex bundle, we have that each $c_{i}$ is even.

\section{Part VII On the torsion of the groups $\operatorname{Cob}_{\tau}\left(P^{n+k}\right)$}

In the previous sections we gave a fairly complete computation of the ranks of the cobordism groups of $\tau$-maps. Here we present an approach to the computation of the torsion part. We shall see that the stable homotopy groups of spheres and the homology rings of the groups $G_{\eta}, \eta \in \tau$ give an upper bound on the torsions of $\operatorname{Cob}_{\tau}(n, k)$. The Thom polynomials $T p_{\eta}$ evaluated on closed manifolds will give an estimation of these torsion groups from below.

The stable Hurewicz homomorphism induces isomorphism not only rationally but also between the $p$-components for sufficiently high primes. Arlettaz [2] showed the following:

Theorem 12 (Arlettaz) For any $(b-1)$-connected spectrum $X$, the stable Hurewicz homomorphism $h_{m}: \pi_{m}(X) \rightarrow H_{m}(X ; Z)$ has the following properties. Let $\varrho_{j}$ be the exponent of $\pi_{j} S=\pi_{j+N}\left(S^{N}\right), N \gg 1$. Then:

a) $\left(\varrho_{1} \ldots \varrho_{m-b}\right)\left(\operatorname{ker} h_{m}\right)=0, \quad \forall m \geq b+1$,

b) $\left(\varrho_{1} \ldots \varrho_{m-b-1}\right)\left(\operatorname{coker} h_{m}\right)=0, \quad \forall m \geq b+2$.

According to Serre [33] the numbers $\varrho_{j}$ are not divisible by a prime $p$ if $p>(1 / 2) j+1$. Let us apply this theorem to (any spectrum defined by) the virtual space $T v^{k}$; see Remark 62. Note that such a spectrum is $(k-1)$-connected. Put $b=k$ and $m=n+k$. Then

$$
h_{n+k}: \pi_{n+k}^{s}\left(T v^{k}\right) \longrightarrow H_{n+k}\left(T v^{k}\right) \approx H_{n}\left(\mathcal{K}_{\tau}\right)
$$

has the property:

$$
\begin{aligned}
\varrho_{1} \ldots \varrho_{n}\left(\operatorname{ker} h_{n+k}\right)=0 & \forall n \geq 1 \\
\varrho_{1} \ldots \varrho_{n-1}\left(\operatorname{coker} h_{n+k}\right)=0 & \forall n \geq 2 .
\end{aligned}
$$

Hence for $p>n / 2+1$ the map $\varphi: \operatorname{Cob}_{\tau}(n, k) \rightarrow H_{n}\left(\mathcal{K}_{\tau}\right)$ induces an isomorphism of the $p$-components $(n \geq 2)$. Analogously for any $(n+k)$-manifold $P^{n+k}$ the map

$$
\varphi: \operatorname{Cob}_{\tau}\left(P^{n+k}\right) \longrightarrow \bigoplus_{j} H^{j}\left(P^{n+k}, H_{j-k}\left(\mathcal{K}_{\tau}\right)\right)
$$


described in Proposition 90 (and after) induces isomorphism of $p$-components for $p>n / 2+1, n \geq 2$. Indeed, Arlettaz' theorem can be reformulated in our case by saying that the map $\Gamma T v^{k} \rightarrow S P T v^{k}$ induces a $p$-homotopy equivalence (ie an isomorphism of the $p$-components of the homotopy groups) until dimension $n+k$ if $p>n / 2+1$. Now the equalities

$$
\begin{aligned}
{\left[P^{n+k}, \Gamma T \nu^{k}\right] } & =\operatorname{Cob}_{\tau}\left(P^{n+k}\right), \\
{\left[P^{n+k}, S P T \nu^{k}\right] } & =\bigoplus_{j} H^{j}\left(P^{n+k}, H_{j-k}\left(\mathcal{K}_{\tau}\right)\right)
\end{aligned}
$$

imply the statement.

Proposition 122 There exists a double spectral sequence

$$
E_{p, q, i}^{1}=H_{p}\left(B G_{i} ; \pi_{q}^{s}\right) \text { converging to } \operatorname{Cob}_{\tau}(n, k) .
$$

Explanation 123 Here - as before $-\tau=\left\{\eta_{0}<\eta_{1}<\cdots<\eta_{i}<\cdots<\eta_{r}\right\}$, $G_{i}$ is the group $G_{\eta_{i}}$ (= maximal compact subgroup of the automorphism group of the root of $\left.\left[\eta_{i}\right]\right)$.

1) The groups $E_{p, q, i}^{1}$ for any fixed $i$ form the initial term of a spectral sequence, which converges to some groups $F_{m, i}(m=p+q)$, and the latter form the initial term of a spectral sequence that converges to $\operatorname{Cob}_{\tau}(n, k)$. (Here $n=m+i$ and $k$ is fixed.)

2) We can get a double spectral sequence from $H_{p}\left(B G_{i} ; \pi_{q}^{S}\right)$ to $\left.\mathrm{Cob}\right|_{\tau}(*, k)$ also in a second way, namely we could start by fixing the index $q$ first.

Proof Let us consider the diagram

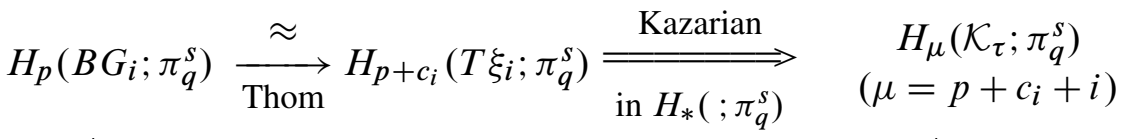

$$
\begin{aligned}
& \approx \downarrow \text { Thom } \\
& \approx \downarrow \text { Thom } \\
& H_{p+c_{i}+k}\left(T \widetilde{\xi}_{i} ; \pi_{q}^{s}\right) \\
& H_{\mu+k}\left(V_{\tau} ; \pi_{q}^{s}\right) \\
& \text { ॥ Atiyah-Hirz. } \\
& \pi_{m}^{s}\left(T \tilde{\xi}_{i}\right) \\
& \text { \Atiyah-Hirz. } \\
& \pi_{*}^{s}\left(V_{\tau}\right)=\pi_{*}\left(X_{\tau}\right)=\operatorname{Cob}_{\tau}(*, k) \\
& \left(m=p+c_{i}+k+q\right) \text {. }
\end{aligned}
$$


The two paths from $H_{p}\left(B G_{i} ; \pi_{q}^{S}\right)$ to $\operatorname{Cob}_{\tau}(*)$ give the two double spectral sequences. The double arrows indicate the corresponding spectral sequences.

Although these spectral sequences give a way in principle to compute the group $\operatorname{Cob}_{\tau}(n, k)$ completely, the practical computation seems to be rather difficult. There are two very special cases when the groups $\operatorname{Cob}_{\tau}(n, k)$ are completely computed.

Theorem 13 [10] Let $\tau$ be $\left\{\Sigma^{0}, \Sigma^{1,0}\right\}$, ie $\tau$-maps are fold maps. Then

$$
\operatorname{Cob}_{\tau}^{S O}(4 m-1,2 m-1) \approx \Omega_{4 m-1} \oplus \mathbb{Z}_{3 t(m)},
$$

where $t(m)=\min \left\{j \mid \alpha_{3}(2 m+j) \leq 3 j\right\}$ and $\alpha_{3}(x)$ denotes the sum of digits of the integer $x$ in triadic system.

In [10] we computed the cobordism groups of fold maps $\operatorname{Cob}_{\tau}(n, k)$ for all cases when $n=2 k-1$. For the case of fold maps of $2 K+2$ manifolds in the Euclidean space $\mathbb{R}^{3 K+2}$ Terpai gave a complete computation in [42].

\section{The (left-right) bordism groups of $\tau$-maps}

By (left-right) bordism of $\tau$-maps we mean the version of $\tau$-cobordism when not only the source but also the target manifold of the $\tau$-map can be changed by an arbitrary oriented cobordism. We shall denote these groups by $\operatorname{Bord}_{\tau}(n)$. More precisely the definition is the following.

Definition 124 Two $\tau$-maps $f_{i}: M_{i}^{n} \rightarrow P_{i}^{n+k}$ for $i=0,1$ are (left-right) bordant if

a) there is a compact oriented manifold $V^{n+1}$ with boundary $\partial V^{n+1}=-M_{0}^{n} \cup$ $M_{1}^{n}$,

b) there is a compact oriented manifold $W^{n+k+1}$ with boundary $\partial W^{n+k+1}=$ $-P_{0}^{n+k} \cup P_{1}^{n+k}$, and

c) there is a $\tau$-map $H: V^{n+1} \rightarrow W^{n+k+1}$, mapping $M_{i}^{n}$ to $P_{i}^{n+k}$ by the map $f_{i}$.

The set of equivalence classes is denoted by $\operatorname{Bord}_{\tau}(n)$. The disjoint union defines a group operation on this set.

Here we compute the ranks of these groups.

Theorem $14 \operatorname{Bord}_{\tau}(n) \approx \Omega_{n+k}\left(X_{\tau}\right)$. 
Proof The proof is completely analogous to the proof of the Pontrjagin-Thom construction of $\tau$-maps.

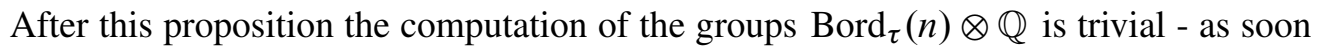
as we suppose the rational homology groups of the Kazarian space to be known. The answer can be formulated in the simplest way using generating functions. ${ }^{15}$

\section{Notation 125}

1) For any space $A$ we denote by $\mathcal{P}_{A}(t)$ the (reduced) rational Poincarè series of the space $A$, ie

$$
\mathcal{P}_{A}(t)=\sum_{i} \mathrm{rk} \tilde{H}_{i}(A, \mathbb{Q}) \cdot t^{i}
$$

2) Let us put

$$
\tau(t)=\sum \operatorname{rk}\left(\operatorname{Bord}_{\tau}(n)\right) \cdot t^{n+k}
$$

Lemma 126 Let us denote by $\mathcal{S P}_{A}(t)$ the rational Poincarè series of the infinite symmetric power $\operatorname{SP}(A)$ of the space $A$. Then $\mathcal{S P}_{A}(t)$ is the following:

$$
\mathcal{S P}_{A}(t)=\prod_{i \text { even }}\left(\frac{1}{1-t^{i}}\right)^{b_{i}(A)} \cdot \prod_{i \text { odd }}\left(1+t^{i}\right)^{b_{i}(A)}
$$

where $b_{i}(A)$ is the $i$-th rational Betti number of the space $A$, and $i$ runs over all natural numbers.

Proof It is well known, that $S P(X)$ is weakly homotopy equivalent to

$$
\bigoplus_{i} K\left(H_{i}(X), i\right)
$$

(see Hatcher [15, page 472]). The cohomology ring of the space $K(\mathbb{Q}, i)$ with rational coefficients is freely generated by an $i$-dimensional multiplicative generator. By the Künneth formula the Poincarè series of a product is the product of those of the factors. All these facts imply the statement easily.

Let us put:

$$
F_{\tau}(t)=\mathcal{S P}_{S^{k}\left(\mathcal{K}_{\tau}^{+}\right)}=\prod_{i \text { even }}\left(\frac{1}{1-t^{i}}\right)^{b_{i-k}\left(\mathcal{K}_{\tau}\right)} \cdot \prod_{i \text { odd }}\left(1+t^{i}\right)^{b_{i-k}\left(\mathcal{K}_{\tau}\right)}
$$

${ }^{15}$ I thank M Kazarian for teaching me the method of generating functions giving a simple way of formulating results. 
Theorem 15 We have the following equality:

$$
\tau(t)=F_{\tau}(t) \cdot \mathcal{P}_{B S O}(t)
$$

Proof Recall that $X_{\tau} \cong \mathbb{Q} S P S^{k}\left(\mathcal{K}_{\tau}^{+}\right)$.

Now using the facts that

$$
\Omega_{i} \otimes \mathbb{Q} \approx H_{i}(B S O ; \mathbb{Q}) \text { and } \Omega_{i}(A) \approx \bigoplus_{a} H_{a}(A) \otimes \Omega_{i-a} \otimes \mathbb{Q}
$$

we obtain the statement of the Theorem.

\subsection{Geometric interpretation of the bordism groups of the Kazarian spaces}

Definition 127 Let us replace in the previous Definition 124 the $\tau$-maps by $\ell$-framed $\tau$-maps, and the target manifolds $P_{i}^{n+k}$ by some closed oriented $n+k+\ell$-dimensional manifolds $Q_{i}^{n+k+\ell}$. The obtained group will be denoted by $\operatorname{Bord}_{\tau \oplus \ell}(n)$.

Proposition 128 For $\ell>n+k$ the group does not depend on $\ell$ and it is isomorphic to the $n$-th bordism group of the space $\mathcal{K}_{\tau}$ :

$$
\operatorname{Bord}_{\tau \oplus \ell}(n) \approx \Omega_{n}\left(\mathcal{K}_{\tau}\right) .
$$

The unoriented analogue of this statement holds as well.

Remark 129 This Proposition can be considered as a geometric interpretation of the homology groups of the Kazarian space because of the formulas connecting homology groups and bordism groups; see Conner and Floyd [9].

Proof We have the following:

$$
\begin{aligned}
\Omega_{n}\left(\mathcal{K}_{\tau}\right) \approx \Omega_{n+k}\left(T \nu^{k}\right) & \approx \Omega_{n+k+\ell}\left(S^{\ell} T \nu^{k}\right) \\
& \approx \Omega_{n+k+\ell}\left(S^{\ell} V_{\tau}\right) \stackrel{(*)}{\approx} \Omega_{n+k+\ell}\left(\Gamma\left(S^{\ell} V_{\tau}\right)\right) \approx \Omega_{n+k+\ell}\left(B^{\ell} X_{\tau}\right)
\end{aligned}
$$

Here $\Gamma$ is the functor $\Omega^{\infty} S^{\infty}$. The isomorphism $(*)$ holds because for any $i-$ connected space $A$ the pair $(\Gamma(A), A)$ is $2 i$-connected [6]. $B^{\ell}$ means the $\ell$-times "deloopization" of the space $X_{\tau}$, ie the space $Z_{\tau}^{\ell}$; see Remark 17 . 


\section{Final remarks, open problems}

\subsection{Cobordism groups of singular maps as extraordinary cohomology theories}

We have seen that all the classifying spaces $X_{\tau}$ and also all the spaces $\Gamma T \widetilde{\xi}_{\eta}$ are infinite loop spaces. Therefore they all determine extraordinary cohomology theories. Namely: For $q \geq 0$ we put

$$
\begin{gathered}
h_{\tau}^{-q}\left(P^{n+k}\right) \stackrel{\text { def }}{=} \operatorname{Cob}_{\tau}\left(P^{n+k} \times \mathbb{R}^{q}\right), \\
h_{\tau}^{q}\left(P^{n+k}\right) \stackrel{\text { def }}{=} \operatorname{Cob}_{\tau \oplus q}\left(P^{n+k}\right) .
\end{gathered}
$$

(Recall that $\operatorname{Cob}_{\tau \oplus q}($ ) denotes the cobordism group of $\tau$-maps with $q$-framing. Recall also that we have extended the functors $\operatorname{Cob}_{\tau}\left(\right.$ ) and $\operatorname{Cob}_{\tau \oplus q}($ ) from manifolds to simplicial complexes.)

If $\alpha: P \rightarrow P^{\prime}$ is any continuous map of simplicial complexes, then the induced homomorphism $\alpha^{*}=h_{\tau}^{q}(\alpha)$ is defined by taking transverse preimages. The maps $\theta_{\tau^{\prime}, \tau}: X_{\tau^{\prime}} \rightarrow X_{\tau}$ or $\theta_{\tau, \eta}: X_{\tau} \rightarrow \Gamma T \tilde{\xi}_{\eta}$ when $\tau^{\prime}$ is a subset of $\tau$, and $\eta$ is a top singularity in $\tau$ define cohomology operations. For example we have seen that $[f] \in \operatorname{Cob}_{\tau}(P)$ contains an $\eta$-free map iff the cohomology operation $\theta_{\tau, \eta}$ vanishes on $[f]$. Extending the list of singularities included in $\tau$ we obtain better and better approximations of the usual cobordism theory

$$
M S O^{-k}(P)=\lim _{N \rightarrow \infty}\left[S^{N+k} P, M S O(N)\right] .
$$

We have computed all these cohomology theories modulo $p$-torsion for "small" primes $p$ - by expressing them through the homologies of the corresponding Kazarian space $\mathcal{K}_{\tau}$, or equivalently through the $S O$-equivariant cohomologies of the space of some polynomial maps $\mathcal{P}_{\tau}=\lim _{N \rightarrow \infty} \mathcal{P}_{\tau}\left(\mathbb{R}^{N}, \mathbb{R}^{N+k}\right)$. (The $S O$-action is one-sided; see the Appendix, Section 20.)

\subsection{Open problems}

1) How to define and compute multiplicative structures on these cohomology theories?

2) Compute the torsion of $\operatorname{Cob}_{\tau}\left(P^{n+k}\right)$ (see Section VII).

3) Extend all the results of this paper to unoriented cobordism groups.

4) Find analogues of exact sequences of Rohlin, Wall, Atiyah relating the oriented and unoriented versions of these groups (see theorems 4.2 and 4.3 in [5]). 


\section{Appendix: The stabilisation of the Kazarian space}

First we recall the Borel construction:

If $V$ is a $G$-space (ie a $G$-action $G \times V \rightarrow V$ is given on it), then the Borel construction of $V$ is the space $B V=E G \times{ }_{G} V$. If $\Xi \subset V$ is a $G$-invariant subspace, then we have $B \Xi \subset B V$.

If $V=\bigcup_{\eta} \Sigma_{\eta}$ is the decomposition of $V$ into $G$-orbits (parameterised by $\eta$ ), then $B V=\bigcup_{\eta} B \Sigma_{\eta}$.

Lemma 130 (Kazarian [22]) If $V$ is contractible, and $G_{x}$ is the stabiliser of a point $x \in V$, and $\Sigma=G(x)$ is the orbit of $x$, then $B \Sigma=B G_{x}$, where $B G_{x}$ is the classifying space of the group $G_{X}$.

Proof $B \Sigma=E G \times_{G} \Sigma=E G \times_{G} G / G_{x}=(E G) / G_{x}=B G_{x}$.

Notation 131 By $\mathcal{K}_{\tau}^{\prime}(n)$ we denoted the "unstable" Kazarian space which was a subset in $J^{K}\left(\gamma_{n}^{S O}, \gamma_{n+k}^{S O}\right)$. Namely: the fibre of the bundle

$$
J^{K}\left(\gamma_{n}^{S O}, \gamma_{n+k}^{S O}\right) \longrightarrow B S O_{n} \times B S O_{n+k}
$$

is $J_{0}^{K}\left(\mathbb{R}^{n}, \mathbb{R}^{n+k}\right)$ of the space of all polynomial maps $\mathbb{R}^{n} \rightarrow \mathbb{R}^{n+k}$ of degree $\leq K$ and mapping the origin of $\mathbb{R}^{n}$ into that of $\mathbb{R}^{n+k}$. Let $V_{\tau}(n) \subset J_{0}^{K}\left(\mathbb{R}^{n}, \mathbb{R}^{n+k}\right)$ consist of the maps having a singularity at the origin from the list $\tau$.

The union of these subsets $V_{\tau}(n)$ in each fibre forms the space $\mathcal{K}_{\tau}^{\prime}(n)$.

Applying the previous Lemma we would get the decomposition $\mathcal{K}_{\tau}^{\prime}(n)=\bigcup B G_{x}$, where $G_{x}$ is the stabiliser group of $x \in V_{\tau}(n)$ under the action of $G=S O_{n} \times S O_{n+k}$. There is a small problem here. Namely that the stabiliser groups are not direct products. Namely if $x$ is representing a singularity class $[\eta]$, then the stabiliser $G_{x}$ is an index 2 subgroup of $G_{\eta}^{O} \times O(n-c(\eta))$, (recall that $c(\eta)$ is the codimension of the $\eta$-stratum in the source). The same group $G_{x}$ can be described also as a $\mathbb{Z}_{2}$ extension of the group $G_{\eta} \times S O(n-c(\eta))$.

In order to obtain the stabiliser subgroups as direct products $G_{\eta} \times O(n-c(\eta))$, we apply the following trick. We define a modification of the unstable Kazarian space $\mathcal{K}_{\tau}^{\prime}(n)$. Let us define the subgroup $H_{n}$ of index 2 in $O_{n} \times O_{n+k}$ as follows:

$$
H_{n}=\left\{(A, B) \mid A \in O_{n}, B \in O_{n+k}, \operatorname{det}(A) \cdot \operatorname{det}(B)>0\right\} .
$$

Put

$$
\mathcal{K}_{\tau}^{\mathrm{co}}(n)=V_{\tau}(n) \times_{H_{n}} E H_{n} .
$$


(This is a version of the unstable Kazarian space $\mathcal{K}_{\tau}^{\prime}(n)$ for the cooriented maps, ie for the maps having orientations on their virtual normal bundles, but not necessarily both on the source and on the target.)

Lemma $132 \mathcal{K}_{\tau}^{\mathrm{co}}(n)=\bigcup_{\eta \in \tau} B G_{\eta} \times B O(n-c(\eta))$.

Proof Let $\eta$ be a singularity class from $\tau$ and let us denote by $\eta^{0}$ the root of $\eta$. Recall that $\eta^{0}$ is a germ $\left(\mathbb{R}^{c(\eta)}, 0\right) \rightarrow\left(\mathbb{R}^{c(\eta)+k}, 0\right)$, and $\eta$ is locally equivalent to $\eta^{0} \times \mathrm{id}_{\mathbb{R}^{n-c(\eta)}}$. Then the automorphism group of $\eta$ in $H_{n}$ is $G_{\eta} \times O(n-c(\eta))$. Hence if $x \in V_{\tau}(n)$ represents $\eta$ then the stabiliser is $G_{x}^{(n)}=G_{\eta} \times O(n-c(\eta))$. Now apply the previous Lemma.

Definition 133 The (stable) Kazarian space is defined as:

$$
\mathcal{K}_{\tau} \stackrel{\text { def }}{=} \lim _{N \rightarrow \infty} E S O(N+k) \underset{S O(N+k)}{\stackrel{\times}{*}} V_{\tau}(N) \subset \lim _{N \rightarrow \infty} J^{K}\left(\varepsilon^{N}, \gamma_{N+k}\right)
$$

Note that here the action of the group $S O(N+k)$ is one sided, we do not act on the source space $\mathbb{R}^{N}$ of the polynomial maps.

Proposition 134 The (stable) Kazarian space $\mathcal{K}_{\tau}$ decomposes into the disjoint union of the base spaces $B G_{\eta}$ for $\eta \in \tau$. (Here $G_{\eta}$ is the maximal compact subgroup of the automorphism group of the roof of $\eta$.)

Proof The double cover $B S O_{n} \times B S O_{n+k} \rightarrow B H_{n}$ induces a double cover $\mathcal{K}_{\tau}^{\prime}(n) \rightarrow$ $\mathcal{K}_{\tau}^{\mathrm{co}}(n)$. Let us denote by $\mathcal{K}_{\tau}(n)$ the lift of the bundle $\mathcal{K}_{\tau}^{\prime}(n) \rightarrow B S O_{n} \times B S O_{n+k}$ to the space $E S O_{n} \times B S O(n+k) \cong B S O_{n+k}$. Clearly the map $\mathcal{K}_{\tau}(n) \rightarrow \mathcal{K}_{\tau}^{\prime}(n)$ is an $S O_{n}$-bundle. The composition of this bundle map with the double cover $\mathcal{K}_{\tau}^{\prime}(n) \rightarrow$ $\mathcal{K}_{\tau}^{\mathrm{co}}(n)$ is an $O_{n}$-bundle. The part of this $O_{n}$ bundle over the stratum $B G_{\eta} \times B O(n-c)$ is the space of cosets $O_{n} /\left(O_{n-c} \times G_{\eta}\right)$, ie the quotient of the Stiefel manifold $V_{c}\left(R^{n}\right)$ by a free $G_{\eta}$ action. Since the Stiefel manifold is $n-c-1$-connected the limit for $n \rightarrow \infty$ will be the classifying space $B G_{\eta}$. Hence the corresponding stratum in the stable Kazarian space $\mathcal{K}_{\tau}$ will be $B G_{\eta}$.

Remark 135 It follows from the construction of the stable Kazarian space $\mathcal{K}_{\tau}$ that any (proper) $\tau$-map defines a homotopically unique map of its source to the space $\mathcal{K}_{\tau}$. 


\section{References}

[1] Y Ando, Cobordism of maps without prescribed singularities arXiv: math.GT/0412234

[2] D Arlettaz, Exponents for extraordinary homology groups, Comment. Math. Helv. 68 (1993) 653-672 MR1241477

[3] V I Arnol' d, S M Guseĭn-Zade, A N Varchenko, Singularities of differentiable maps. Vol. I. The classification of critical points, caustics and wave fronts, Monographs in Math. 82, Birkhäuser, Boston (1985) MR777682 Translated from the Russian by I Porteous and M Reynolds

[4] V I Arnol' d, V A Vasil' ev, V V Goryunov, O V Lyashko, Singularities. I. Local and global theory, from: "Current problems in mathematics. Fundamental directions, Vol. 6”, Itogi Nauki i Tekhniki, Akad. Nauk SSSR Vsesoyuz. Inst. Nauchn. i Tekhn. Inform., Moscow (1988) 5-257 MR1088739

[5] M F Atiyah, Bordism and cobordism, Proc. Cambridge Philos. Soc. 57 (1961) 200-208 MR0126856

[6] M G Barratt, P J Eccles, $\Gamma^{+}$-structures. I. A free group functor for stable homotopy theory, Topology 13 (1974) 25-45 MR0348737

[7] O Burlet, Cobordismes de plongements et produits homotopiques, Comment. Math. Helv. 46 (1971) 277-288 MR0295367

[8] D S Chess, Singularity theory and configuration space models of $\Omega^{n} S^{n}$ of nonconnected spaces, Topology Appl. 25 (1987) 313-338 MR889874

[9] P E Conner, E E Floyd, Differentiable periodic maps, Ergebnisse series 33, Springer, New York (1964) MR0176478

[10] T Ekholm, A Szúcs, T Terpai, Cobordisms of fold maps and maps with a prescribed number of cusps, Kyushu J. Math. 61 (2007) 395-414 MR2362892

[11] J Eliashberg, Cobordisme des solutions de relations différentielles, from: "South Rhone seminar on geometry, I (Lyon, 1983)", Travaux en Cours, Hermann, Paris (1984) 17-31 MR753850

[12] R Godement, Topologie algébrique et théorie des faisceaux, Actualit'es Sci. Ind. 1252, Publ. Math. Univ. Strasbourg 13, Hermann, Paris (1958) MR0102797

[13] M Gromov, Partial differential relations, Ergebnisse series 9, Springer, Berlin (1986) MR864505

[14] A Haefliger, A Kosiński, Un théorème de Thom sur les singularités des applications différentiables, from: "Séminaire Henri Cartan; 9e année: 1956/57. Quelques questions de topologie, Exposé no. 8”, Secrétariat mathématique, Paris (1958) 6 MR0124063

[15] A Hatcher, Algebraic topology, Cambridge University Press (2002) MR1867354

[16] D Husemoller, Fibre bundles, McGraw-Hill, New York (1966) MR0229247 
[17] K Ikegami, Cobordism group of Morse functions on manifolds, Hiroshima Math. J. 34 (2004) 211-230 MR2086843

[18] K Ikegami, O Saeki, Cobordism group of Morse functions on surfaces, J. Math. Soc. Japan 55 (2003) 1081-1094 MR2003761

[19] K Jänich, Symmetry properties of singularities of $C^{\infty}$-functions, Math. Ann. 238 (1978) 147-156 MR512820

[20] B Kalmár, Cobordism group of Morse functions on unoriented surfaces, Kyushu J. Math. 59 (2005) 351-363 MR2188598

[21] M Kazarian, private communication

[22] M Kazarian, Classifying spaces of singularities and Thom polynomials, from: "New developments in singularity theory (Cambridge, 2000)", NATO Sci. Ser. II Math. Phys. Chem. 21, Kluwer Acad. Publ., Dordrecht (2001) 117-134 MR1849306

[23] M Kazarian, Characteristic classes in singularity theory, Habilitation thesis, Moscow (2003) Available at http://www.mi.ras.ru/ kazarian/publ

[24] U Koschorke, Vector fields and other vector bundle morphisms-a singularity approach, Lecture Notes in Math. 847, Springer, Berlin (1981) MR611333

[25] G Lippner, A Szúcs, Multiplicative properties of Morin maps arXiv: math.GT:0710.2681

[26] J N Mather, Stability of $C^{\infty}$ mappings. IV. Classification of stable germs by $R-$ algebras, Inst. Hautes Études Sci. Publ. Math. (1969) 223-248 MR0275460

[27] J W Milnor, J D Stasheff, Characteristic classes, Annals of Math. Studies 76, Princeton University Press (1974) MR0440554

[28] R E Mosher, M C Tangora, Cohomology operations and applications in homotopy theory, Harper \& Row Publishers, New York (1968) MR0226634

[29] R Rimányi, A Szúcs, Pontrjagin-Thom-type construction for maps with singularities, Topology 37 (1998) 1177-1191 MR1632908

[30] C Rourke, B Sanderson, The compression theorem. I, Geom. Topol. 5 (2001) 399-429 MR1833749

[31] R Sadykov, Bordism groups of solutions of differential relations arXiv: math. AT/0608460

[32] O Saeki, Topology of singular fibers of differentiable maps, Lecture Notes in Math. 1854, Springer, Berlin (2004) MR2106689

[33] J-P Serre, Groupes d'homotopie et classes de groupes abéliens, Ann. of Math. (2) 58 (1953) 258-294 MR0059548

[34] A Szúcs, Analogue of the Thom space for mappings with singularity of type $\Sigma^{1}$, Mat. Sb. (N.S.) 108 (150) (1979) 433-456, 478 MR530320 
[35] A Szúcs, Cobordism of maps with simplest singularities, from: "Proc. Sympos., Univ. Siegen, 1979)", Lecture Notes in Math. 788, Springer, Berlin (1980) 223-244 MR585661

[36] A Szúcs, Cobordism of immersions and singular maps, loop spaces and multiple points, from: "Geometric and algebraic topology", Banach Center Publ. 18, PWN, Warsaw (1986) 239-253 MR925868

[37] A Szúcs, On the cobordism groups of immersions and embeddings, Math. Proc. Cambridge Philos. Soc. 109 (1991) 343-349 MR1085401

[38] A Szúcs, Topology of $\Sigma^{1,1}$-singular maps, Math. Proc. Cambridge Philos. Soc. 121 (1997) 465-477 MR1434655

[39] A Szúcs, On the cobordism group of Morin maps, Acta Math. Hungar. 80 (1998) 191-209 MR1645258

[40] A Szúcs, Elimination of singularities by cobordism, from: "Real and complex singularities”, Contemp. Math. 354, Amer. Math. Soc. (2004) 301-324 MR2088057

[41] A Szúcs, Cobordism of singular maps arXiv:math.GT/0612152v1

[42] T Terpai, Cobordisms of fold maps of $(2 K+2)-$ manfolds into $\mathbb{R}^{3 K+2}$, to appear in Banach Center Publications

[43] R Thom, Quelques propriétés globales des variétés différentiables, Comment. Math. Helv. 28 (1954) 17-86 MR0061823

[44] C T C Wall, A second note on symmetry of singularities, Bull. London Math. Soc. 12 (1980) 347-354 MR587705

[45] R Wells, Cobordism groups of immersions, Topology 5 (1966) 281-294 MR0196760

Eötvös Loránd University, Pázmány Péter sétány 1/C, 3-206

1117 Budapest, Hungary

szucs@cs.elte.hu

Proposed: Yasha Eliashberg

Seconded: Ralph Cohen, Steve Ferry
Received: 8 September 2006 Revised: 27 June 2008 